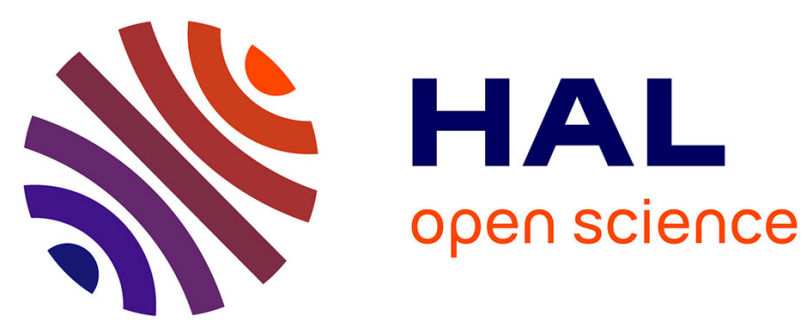

\title{
Geochronology and isotope analysis of the Late Paleozoic to Mesozoic granitoids from Northeastern Vietnam and implications for the evolution of the South China block
}

Zechao Chen, Wei Lin, Michel Faure, Claude Lepvrier, Nguyen van Vuong, Vu van Tich

\section{To cite this version:}

Zechao Chen, Wei Lin, Michel Faure, Claude Lepvrier, Nguyen van Vuong, et al.. Geochronology and isotope analysis of the Late Paleozoic to Mesozoic granitoids from Northeastern Vietnam and implications for the evolution of the South China block. Journal of Southeast Asian earth sciences, 2014, 86, pp.131-150. 10.1016/j.jseaes.2013.07.039 . insu-00858117

\section{HAL Id: insu-00858117 https://hal-insu.archives-ouvertes.fr/insu-00858117}

Submitted on 13 Dec 2013

HAL is a multi-disciplinary open access archive for the deposit and dissemination of scientific research documents, whether they are published or not. The documents may come from teaching and research institutions in France or abroad, or from public or private research centers.
L'archive ouverte pluridisciplinaire HAL, est destinée au dépôt et à la diffusion de documents scientifiques de niveau recherche, publiés ou non, émanant des établissements d'enseignement et de recherche français ou étrangers, des laboratoires publics ou privés. 


\title{
Geochronology and isotope analysis of the Late Paleozoic to Mesozoic granitoids from northeastern Vietnam and implications for the evolution of the South China block
}

- Zechao Chen ${ }^{\mathrm{a}, \mathrm{e}}$

- Wei $\operatorname{Lin}^{\mathrm{a}}$

- Michel Faure ${ }^{\mathrm{b}}$

- Claude Lepvrier ${ }^{\mathrm{c}}$

- Nguyen Van Vuong ${ }^{\mathrm{d}}$

- Vu Van Tich ${ }^{\mathrm{d}}$

- $\quad{ }^{a}$ State Key Laboratory of Lithospheric Evolution, Institute of Geology and Geophysics, Chinese Academy of Sciences, Beijing, China

- ${ }^{\mathrm{b}}$ Institut des Sciences de la Terre d'Orléans (ISTO), UMR CNRS 6113, Université d'Orléans, 45067 Orléans Cedex 2, France

- $\quad{ }^{\mathrm{c}}$ Institut des Sciences de la Terre de Paris (ISTeP), UMR CNRS 7193, Case 129, Université Pierre \& Marie Curie, 4 Place Jussieu, 75252 Paris Cedex 05, France

- ${ }^{\mathrm{d}}$ Faculty of Geology, Hanoi University of Science, 334 Nguyen Trai, Thanh Xuan, Hanoi, Viet Nam

- ${ }^{\mathrm{e}}$ Graduate University of Chinese Academy of Sciences, Beijing 100049, China

\begin{abstract}
In northeastern Vietnam, Late Paleozoic and Permo-Triassic granitic plutons are widespread, but their tectonic significance is controversial. In order to understand the regional magmatism and crustal evolution processes of the South China block (SCB), this study reports integrated in situ $\mathrm{U}-\mathrm{Pb}, \mathrm{Hf}-\mathrm{O}$ and $\mathrm{Sr}-\mathrm{Nd}$ isotope analyses of granitic rocks from five plutons in northeastern Vietnam. Zircon SIMS U-Pb ages of six granitic samples cluster around in two groups $255-228 \mathrm{Ma}$ and $90 \mathrm{Ma}$. Bulk-rock $\varepsilon_{\mathrm{Nd}}(t)$ ranges from -11 to -9.7 , suggesting that continental crust materials were involved in their granitic genesis. In situ zircon $\mathrm{Hf}-\mathrm{O}$ isotopic measurements for the granitic samples yield a mixing trend between the mantle- and supracrustal-derived melts. It is suggested that the granitic rocks were formed by re-melting of the continental crust. These new data are compared with the Paleozoic and Mesozoic granitic rocks of South China. We argue that northeastern Vietnam belongs to the South China block. Though still speculated, an ophiolitic suture between NE Vietnam and South China, so-called Babu ophiolite, appears unlikely. The Late Paleozoic to Mesozoic magmatism in the research area provides new insights for the magmatic evolution of the South China block.
\end{abstract}

Keywords : Northeastern Vietnam; South China block; Triassic orogen; Zircon SIMS dating; Hf-O isotopes; Eastern Paleotethys 


\section{Introduction}

Southeastern Eurasia is an important part of the tectonic framework in the continental margin of eastern Asia and it was considered as the result of collision or accretion processes by several micro-continents with the South China block (SCB) during Permian-Triassic ca. 270 $240 \mathrm{Ma}$ (Nagy et al., 2001, Osanai et al., 2001, Osanai et al., 2006, Lan et al., 2003, Nakano et al., 2008, Lepvrier et al., 2008 and Sone and Metcalfe, 2008). As one of the largest blocks on southeastern Eurasia, the SCB is composed of the Yangtze craton to the northwest and the Cathaysia block in the southeast, respectively (Shui, 1987, Yu et al., 2006, Yu et al., 2007 and Wang et al., 2012a). These two blocks were welded together during the Neoproterozoic Jiangnan collision formed at ca. 970-820 Ma (Huang, 1978, Zhang et al., 1984, Shu et al., 1994, Li, 1999, Wu et al., 2006b and Li et al., 2009a, and references therein). The Jiangshan-Shaoxing Fault represents the ophiolitic suture between the Yangtze and Cathaysia blocks (Zhou and Zhu, 1993 and Shu et al., 2008b). From the Late Neoproterozoic to the late Early Paleozoic, the SCB underwent a continuous sedimentation, partly controlled by rifting until the Late Ordovician (Wang and Li, 2003). Since Silurian, the SCB experienced several tectono-thermal events during Late Silurian-Early Devonian, Late Permian-Triassic and Late Mesozoic in different regions (Chen, 1999, Zhou and Li, 2000, Wang et al., 2005, Zhou et al., 2006, Li and Li, 2007, Liang et al., 2008, Faure et al., 2009 and Chu and Lin, this volume). The Late Silurian-Early Devonian event is sealed by a Middle Devonian angular unconformity and the intrusion of Silurian granitoids in the southern part of South China (Huang et al., 1980, JBGMR, 1984, HBGMR, 1988, Yan et al., 2006, Wang et al., 2007c and Wang et al., 2011). The Early Paleozoic orogenic belt is well developed south of the Jiangshan-Shaoxing Fault (Wang et al., 2007c, Faure et al., 2009, Li et al., 2010d and Charvet et al., 2010). It is an intracontinental orogenic belt characterized by southdirected structures, followed by syn- to post- tectonic crustal melting (Lin et al., 2008 and Faure et al., 2009).

The most important tectonic event experienced by the SCB took place in the Early Mesozoic, as recognized by a Late Triassic unconformity widespread across the SCB (Huang et al., 1980, GXBGMR, 1982, JBGMR, 1984 and HBGMR, 1988). Permian-Triassic orogenic belts are well developed around the block, such as Qinling-Dabieshan-Sulu (Hacker et al., 1998, Faure et al., 1999 and Leech and Webb, 2012 and references therein), Songpan-Ganzi (Roger et al., 2010 and Yan et al., 2011), Sanjiang Tethyan (Jian et al., 1999, Wang et al., 2000 and Hou et al., 2007 and reference therein), NE Vietnam (Lepvrier et al., 2011). The Jurassic and Cretaceous tectonic events in the SCB are expressed by granitic intrusions, acidic and intermediate volcanism, NE-SW trending normal or strike-slip faults, over-thrusts, extensional doming, and syn-tectonic terrigeneous sedimentation ( Xu et al., 1987, Gilder et al., 1991, Faure et al., 1996, Lin et al., 2000, Wang et al., 2001, Li et al., 2001, Yan et al., 2003, Zhou et al., 2006 and Shu et al., 2008a). These Late Mesozoic tectonics were interpreted as the result of the subduction of a Paleo-Pacific oceanic plate beneath the Eurasia continent ( John et al., 1990, Charvet et al., 1994, Faure et al., 1996, Zhou et al., 2006 and Li and Li, 2007Jahn). Geochemical studies of the Mesozoic mafic rocks east of the Xuefengshan fault suggest a continental rifting or intracontinental lithospheric extension in response to an upwelling of asthenosphere around $170 \mathrm{Ma}$ ( $\mathrm{Li}$ et al., 2004). However, the compressional deformation events during the Late Triassic to Middle Jurassic within the Cathaysia block ( Charvet et al., 1994 and Chen, 1999) and Late Jurassic to Cretaceous within the southeastern part of Yangtze craton (Yan et al., 2003) make this interpretation unlikely. Therefore the Late Mesozoic tectonic evolution of the SCB is still controversial. 
Granitoids occupy more than half of the surface of the southeastern part of the SCB (Fig. 1. GDBGMR, 1982, JBGMR, 1984, FBGMR, 1985, HBGMR, 1988 and ZBGMR, 1989). In order to understand the tectonic significance of these granitoids, many geochronological and geochemical works have been realized on these granitic rocks (Zhou and Li, 2000, Li et al., 2006, Wang et al., 2007b, Wang et al., 2007c, Li and Li, 2007 and Zhao et al., 2010; Cheng and Mao, 2010 and Chen et al., 2011). The Early Paleozoic granitoids are mostly distributed along Wuyi-Yunkai-Songchay massifs (JBGMR, 1984 and HBGMR, 1988). These plutons can be subdivided into gneissic and massive massifs defined on the basis of presence or absence of a well-developed magmatic fabric (JBGMR, 1984; HBGMR, 1988; Roger et al., 2000 and Wang et al., 2011). Available data show the age of these granitoids mostly range around 460-420 Ma with a per-aluminous character (Charvet et al., 2010, Wan et al., 2010, Li et al., 2010d, Wang et al., 2011 and Wang et al., 2012b). The Late Permian to Triassic granitoids mostly crop out in the Wuyi-Yunkai, Xuefengshan, Darongshan, and southern margin of SCB (Hainan island and NE Vietnam; Fig. 1; Zhou et al., 2006 and Li and Li, 2007). Previous studies of Late Permian-Triassic granitoids in the SCB indicated the majority of these granites has been classified as per-aluminous, and defined as S-type granites containing aluminous rich minerals such as muscovite, garnet and tourmaline (Chen and Jahn, 1998, Wang et al., 2002, Deng et al., 2004, Wang et al., 2005, Zhou et al., 2006, Wang et al., 2007b, Sun et al., 2004, Zhou et al., 2006 and Wang et al., 2007c, and references therein). The other plutons are Permian calc-alkaline I-type granite cropping out in Hainan Island ( Li et al., 2006 and $\mathrm{Li}$ and $\mathrm{Li}, 2007$, and references therein), and $A$-type granites (Sun et al., 2011). The Jurassic and Early Cretaceous granitoids are distributed at the eastern part of the SCB, along the Qinling-Dabieshan orogenic belt and Nanling belt (Fig. 1). Li and Li (2007) argue that these Jurassic to Early Cretaceous plutons correspond to synorogenic magmatism. They exhibit a younging trend toward the craton interior. Late Cretaceous granitic and volcanic rocks occupy most of the SE part of the SCB where they distribute parallel to the coastline (Fig. 1, Zhou and Li, 2000). The Late Mesozoic granitoids, synchronous mafic and ultramafic plutons constitute a bimodal magmatic association assumed to be controlled by lithospheric extension and asthenosphere upwelling within the eastern SCB ( Chen and Zhu, 1993, Suo et al., 1999, Yan et al., 2006, Liang et al., 2008, Chen et al., 2008, Liu et al., 2010 and Wei et al., this volume). The origin of this magmatism is related to the Paleo-Pacific subduction ( Wang et al., 2005 and $\mathrm{Li}$ and $\mathrm{Li}, 2007$ ). 


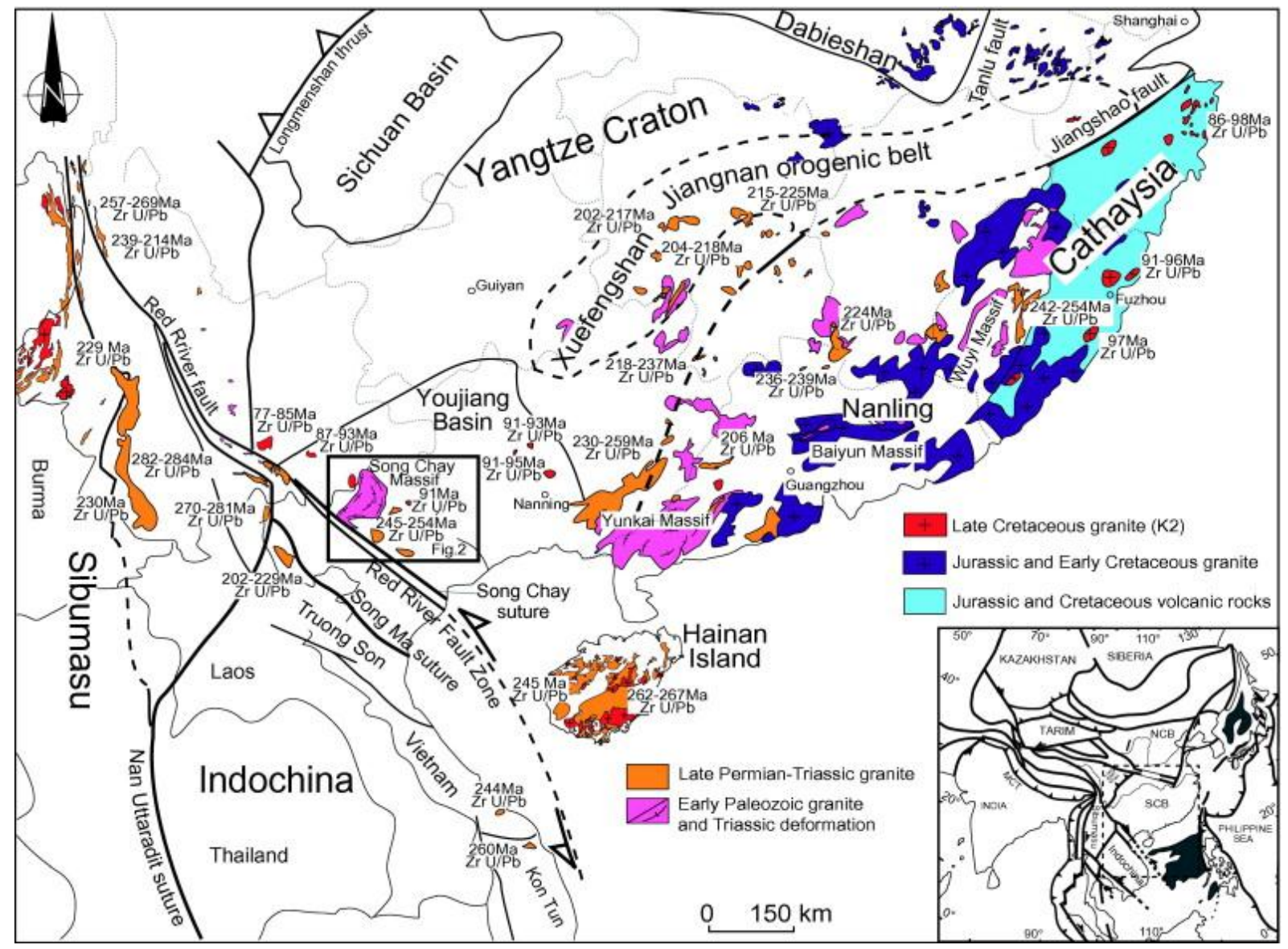

Fig. 1.

Geological map of South China and Indochina blocks, with the emphasis of the Paleozoic and Mesozoic magmatic rocks. Geochronological data from Li et al., 2006, Li, 1994, Li, 1999, Roger et al., 2000, Roger et al., 2012, Zhou and Li, 2000, Jian et al., 2003, Deng et al., 2004, Wang et al., 2005, Cai et al., 2006, Xie et al., 2006, Yan et al., 2006, Zhou et al., 2006, Li and Li, 2007, Liu et al., 2007, Wang et al., 2007a, Tran et al., 2008, Qiu et al., 2008, Tan et al., 2008 and Chen et al., 2009b; Chen et al., 2011, Liu et al., 2010, He et al., 2011 and Chu et al., 2012.

Granitic plutons are less abundant in the southwestern part of the SCB than in its eastern part. The majority of these plutons are dated of Late Permian to Triassic, and Late Cretaceous. In NE Vietnam, the works dealing with this Mesozoic plutonism are still rare (Fig. 2; Tran et al., 2008, Wang et al., 2011, Roger et al., 2000 and Roger et al., 2012), even though this area also belongs to the SCB (Lepvrier et al., 2011). Several questions arise, namely: (i) which tectonic event caused this granitic magmatism? (ii) are the NE Vietnam plutons comparable with those distributed in the eastern part of the SCB? This paper provides new SIMS U-Pb and isotopic data from different plutonic intrusions of NE Vietnam that will allow us to define the plutonic evolution of the southwestern margin of the SCB and discuss its tectonic significance. 


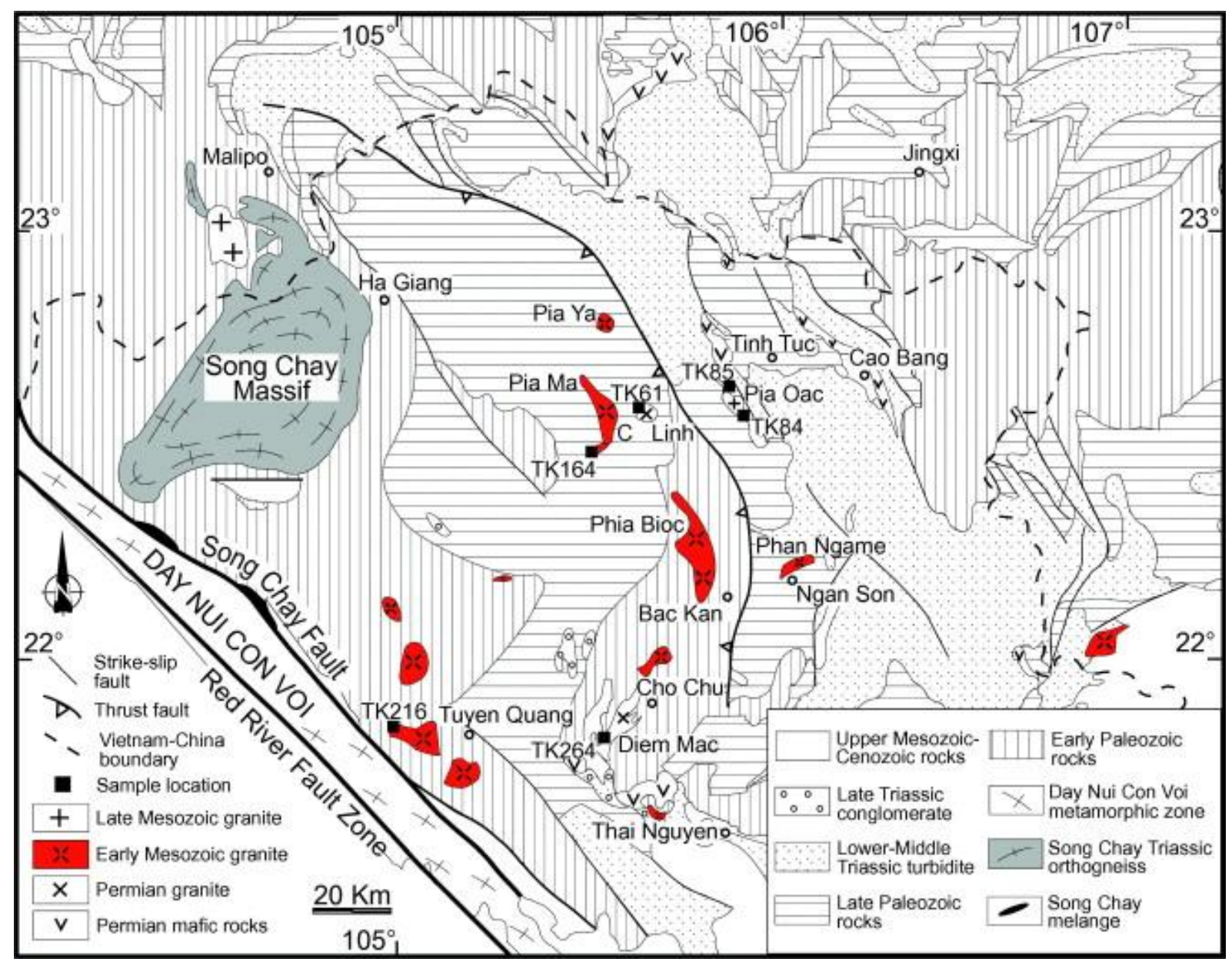

Fig. 2. : Simplified geologic map of northeastern Vietnam and adjacent area showing the locations of the dated sample. 


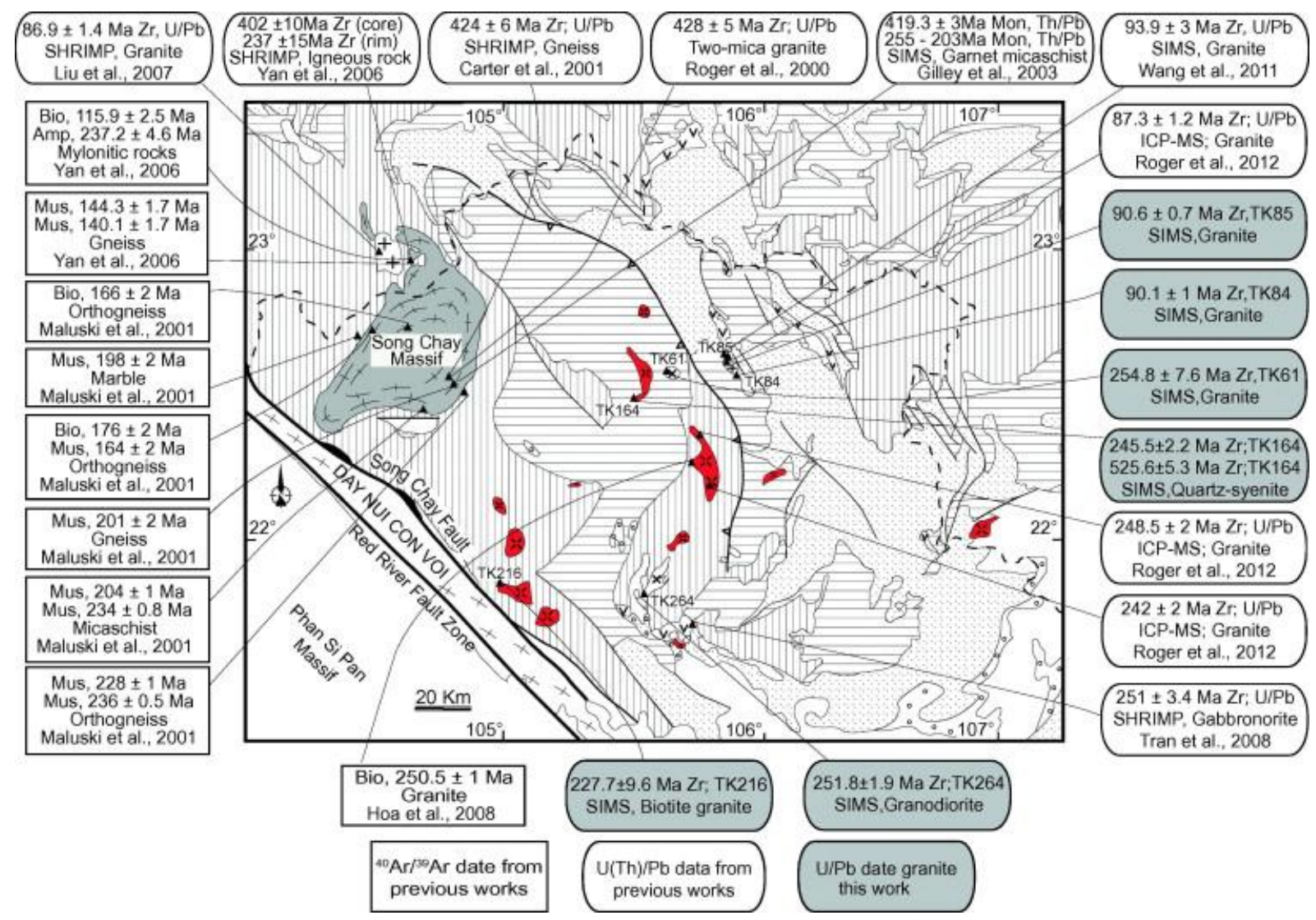

Fig. 3. : Map of the northern Vietnam and its adjacent area showing the available radiometric data. Six SIMS U/Pb ages of zircon are given in this paper. Symbols and captions in the map are the same as in Fig. 2. 

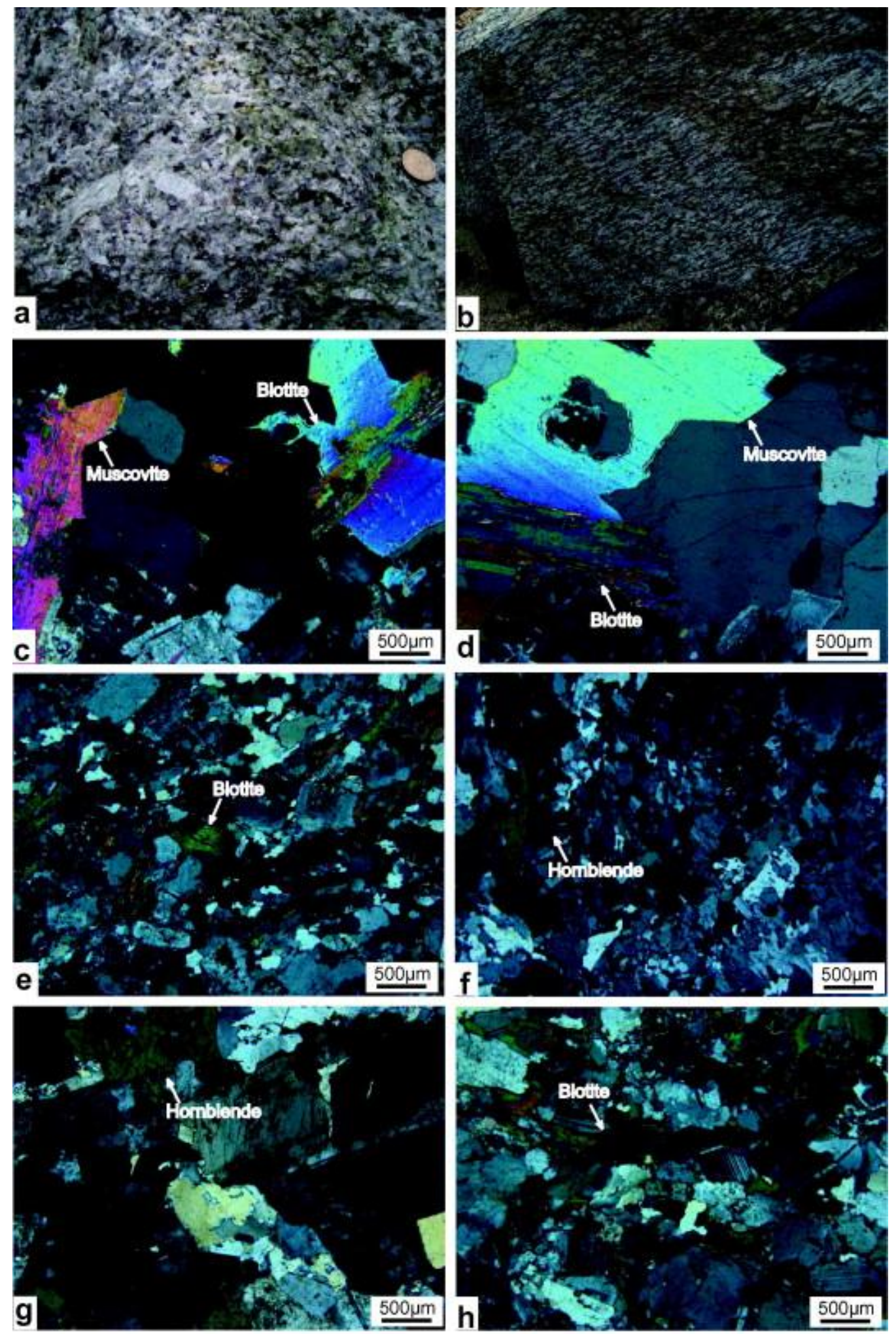

Fig. 4. : Photographs showing the character of granitic samples in northeastern Vietnam. (a) Leucogranites of Pia Oac pluton; (b) Pia Ma quartz-syenite foliated and lineated; (c) Microscope picture of sample TK84 two mica leucogranite; (d) Microscope picture of sample TK85 two mica leucogranite; (e) Microscope picture of sample TK216 biotite granite; $f$. Microscope picture of sample TK164 quartz-syenite; (g) Microscope picture of sample TK264 granodiorite; (h) Microscope picture of sample TK61 granodiorite. 


\section{Geological outline of the NE Vietnam}

The southern boundary of the study area is the Red River Fault Zone (Fig. 2). This major leftlateral ductile shear zone with accommodated several hundreds of kilometers the southeastward during Oligo-Miocene extrusion of Sundaland and acted as a right-lateral fault from Late Pliocene (Tapponnier et al., 1990, Yang and Besse, 1993, Leloup et al., 1995 and Phan et al., 2012). The northern part of study area concerns Chinese Guangxi and Yunnan provinces where the stratigraphy is comparable with northeastern Vietnam (Fig. 2 and Table 1). In ascending stratigraphic order, six lithological and partly metamorphic series have been recognized, namely: (1) Neoproterozoic - Early Paleozoic terrigenous and carbonate sedimentary rocks, deposited in a shallow marine environment; (2) unmetamorphosed but strongly folded Devonian to Permian limestone, siliceous limestone, and some terrigenous rocks; (3) Lower to Middle Triassic turbiditic sediments (conglomerates, sandstones, tuffaceous sandstones, siltstones, shales) with rare carbonates; (4) Upper Triassic continental molassic formation that covers unconformably the previous series; (5) Late Mesozoic continental terrigenous red sandstone; (6) Cenozoic deposits in half-graben or rhombgraben basins along the Song Chay Fault (Fig. 2). 
Table 1. : Stratigraphic column of study area.

\begin{tabular}{|c|c|c|c|c|c|c|}
\hline Epoch & Litho unite & $\begin{array}{c}\text { Thicknes } \\
\text { s (m) }\end{array}$ & $\begin{array}{c}\text { Deformatio } \\
\text { n episode } \\
\text { (Ma) }\end{array}$ & $\begin{array}{c}\text { Tectonic } \\
\text { regime }\end{array}$ & $\begin{array}{l}\text { Intrusive } \\
\text { Event }\end{array}$ & $\begin{array}{c}\text { Tectonic } \\
\text { interpretation }\end{array}$ \\
\hline Quaternary & $\begin{array}{l}\text { Pebble, } \\
\text { granule, sand, } \\
\text { silty clay }\end{array}$ & $24-31$ & & & & \\
\hline Neogene & $\begin{array}{l}\text { Conglomerat } \\
\text { e, gritstone, } \\
\text { sandstone, } \\
\text { Clay shale, } \\
\text { coaly shale }\end{array}$ & $600-800$ & & & & \\
\hline Paleogene & $\begin{array}{l}\text { Conglomerat } \\
\text { e, sandstone, } \\
\text { siltstone, } \\
\text { Coaly shale, } \\
\text { seams and } \\
\text { lenses of } \\
\text { lignite }\end{array}$ & $959-2464$ & & Extension & & $\begin{array}{l}\text { Regional } \\
\text { extension in } \\
\text { east Asia }\end{array}$ \\
\hline Late Cretaceous & $\begin{array}{l}\text { Conglomerat } \\
\text { e, gritstone, } \\
\text { sandstone }\end{array}$ & $200-300$ & & Extension & $\begin{array}{l}\text { Two-Mica } \\
\text { Granite (91- } \\
90 \mathrm{Ma})\end{array}$ & $\begin{array}{l}\text { Regional } \\
\text { extension? }\end{array}$ \\
\hline Jurassic & $\begin{array}{l}\text { Sandy } \\
\text { siltstone, } \\
\text { rhyolite, } \\
\text { gritstone }\end{array}$ & $\begin{array}{l}1650- \\
2000\end{array}$ & & $\begin{array}{l}\text { Extension } \\
?\end{array}$ & & $\begin{array}{l}\text { Regional } \\
\text { extension? }\end{array}$ \\
\hline Late Triassic & $\begin{array}{l}\text { Conglomerat } \\
\text { e, sandstone, } \\
\text { clay shale, } \\
\text { Coaly shale } \\
\text { and coal }\end{array}$ & $\begin{array}{l}1200 \\
1350\end{array}$ & $237-228 \mathrm{Ma}$ & & $\begin{array}{l}\text { Biotite granite } \\
227.7 \pm 9.6 \mathrm{Ma}\end{array}$ & \\
\hline Early-middle & $\begin{array}{l}\text { Quartz } \\
\text { porphyry and } \\
\text { their tuffs, } \\
\text { Sandy } \\
\text { siltstone, } \\
\text { conglomerate } \\
\text {, clay shale }\end{array}$ & $\begin{array}{l}2040- \\
2410\end{array}$ & & Thrusting & $\begin{array}{l}\text { Quartz-syenite } \\
(245.5 \pm 2.2 \mathrm{Ma} \\
)\end{array}$ & $\begin{array}{l}\text { Collision } \\
\text { between } \\
\text { Indochina and } \\
\text { SCB }\end{array}$ \\
\hline 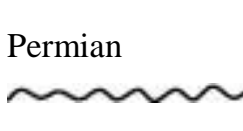 & $\begin{array}{l}\text { Thick bedded } \\
\text { limestone, } \\
\text { cherty shale }\end{array}$ & $100-245$ & & & $\begin{array}{l}\text { Granodiorite } \\
(255-251 \mathrm{Ma})\end{array}$ & Subduction \\
\hline Carboniferous & Limestone & $350-600$ & & & & \\
\hline Devonian & $\begin{array}{l}\text { Sandstone, } \\
\text { shale, } \\
\text { limestone }\end{array}$ & $\begin{array}{l}5680 \\
6330\end{array}$ & & & & \\
\hline Silurian & $\begin{array}{l}\text { Mudstone, } \\
\text { sandstone }\end{array}$ & 400 & & $?$ & $\begin{array}{l}\text { Porphyritic } \\
\text { granite (430- } \\
420 \mathrm{Ma})\end{array}$ & $\begin{array}{l}\text { Intracontinent } \\
\text { al Orogeny }\end{array}$ \\
\hline Ordovician & $\begin{array}{l}\text { Clayish } \\
\text { limestone, } \\
\text { quartzitic } \\
\text { sandstone }\end{array}$ & $\begin{array}{l}2500- \\
2600\end{array}$ & & & & \\
\hline Cambrian & $\begin{array}{l}\text { Shale, } \\
\text { limestone }\end{array}$ & $\begin{array}{l}2000 \\
2300\end{array}$ & & & $\begin{array}{l}\text { Quartz-syenite } \\
(525.6 \pm 5.3 \mathrm{Ma} \\
)\end{array}$ & \\
\hline
\end{tabular}


Paleozoic to Mesozoic granitic plutons intrude into the sedimentary succession (Fig. 2). The Song Chay massif that lies on the western part of the study area in northeastern Vietnam, is an augen-gneiss derived from a porphyritic monzogranite emplaced at $428 \pm 5 \mathrm{Ma}$, according to U-Pb zircon age (Roger et al., 2000). The Phan Ngame orthogneiss that occupies the central part of the Ngan Son antiform (Bourret, 1922 and Fromaget, 1941) is equivalent to the Song Chay orthogneiss. It has been dated at $438.7 \pm 3.5 \mathrm{Ma}$ (Tran and Halpin, 2011). Furthermore, from north to south, there are several km-sized granitic plutons, namely the Pia Ya granitic pluton, Pia Ma quartz-syenite pluton, Có Linh pluton, the Pia Oac leucocratic monzonite granite to the south of Tinh Tuc, the Phia Bioc massif locates in NW of Bac Kan (Fig. 2). Some granitic plutons outcrop along a NW-SE trend north of the Song Chay Fault (Fig. 2). The Phia Bioc granite is porphyritic and undeformed, containing microdioritic enclaves (Roger et al., 2012). This pluton cross-cuts Lower Triassic rocks but occurs as pebbles in the basal conglomerates of the Ladinian (242-235 Ma) sedimentary formation. It yields K-Ar ages scattered from $306 \mathrm{Ma}$ to $230 \mathrm{Ma}$ (Tri, 1979). Recently, this granitic pluton yielded zircon LA-ICPMS U-Pb ages scattered from $247 \mathrm{Ma}$ to $242 \mathrm{Ma}$ (Roger et al., 2012). The undeformed Pia Oac leucocratic monzonitic granite (Bourret, 1922), yields zircon U-Pb SIMS and LA-ICPMS ages of 94-87 Ma (Wang et al., 2011 and Roger et al., 2012). Alkaline mafic rocks that crop out near Cao Bang, west of Tinh Tuc and west of Thai Nguyen, are dated at 266-251 Ma (Tran et al., 2008). This Permian alkaline magmatism is regarded as being produced under the influence of the Emeishan mantle plume (Hanski et al., 2004 and Tran et al., 2008). The Late Permian to Triassic plutons were related to active continental margin magmatism (Liu et al., 2012) and intra-plate magmatism (Tran et al., 2008 and Roger et al., 2012), with the debates on the nature of subduction between SCB and Indochina blocks and suture zones (Lepvrier et al., 2008 and Lepvrier et al., 2011; Liu et al., 2012).

The boundary between the Indochina and South China blocks is generally considered to correspond to the Song Ma ophiolitic suture formed after a north directed subduction (Sengör et al., 1988, Metcalfe, 2002, Lepvrier et al., 1997, Lepvrier et al., 2004 and Lepvrier et al., 2008). However, recent works argue that the SCB subducted beneath Indochina block along the Song Chay suture zone (Lepvrier et al., 2011 and Lin et al., 2011). Following Deprat (1915) and Lepvrier et al. (2011), a stack of nappes, and NE-verging recumbent folds characterize the structure of NE Vietnam.

In order to define precisely the time of this NE-verging synmetamorphic deformation, ${ }^{40} \mathrm{Ar}-$ ${ }^{39} \mathrm{Ar}$ analyses were realized from gneiss and micaschist of the Song Chay massif. Biotite, muscovite and amphibole yield a large time span from $237 \mathrm{Ma}$ to $115 \mathrm{Ma}$. These ages are interpreted as related to slow to moderate uplift in the Late Mesozoic, after the Triassic nappe stacking (Roger et al., 2000, Maluski et al., 2001 and Yan et al., 2006). From monazite inclusions in garnet, an age of 255-203 Ma was obtained by U-Th-Pb method (Gilley et al., 2003). All the available geochronological results obtained in the Song Chay massif are rather consistent but with a large time span. This led some geologists to consider the existence of a long thermal event during the early Mesozoic (Roger et al., 2000 and Carter et al., 2001). However, as the ${ }^{40} \mathrm{Ar} /{ }^{39} \mathrm{Ar}$ method is very sensitive to temperature, it does not appear a suitable method to discriminate the early tectonic and thermal events. 


\section{Sampling and analytical methods}

\subsection{Sample descriptions}

All the six granitic samples come from the NE Vietnam (Fig. 2). Samples TK84

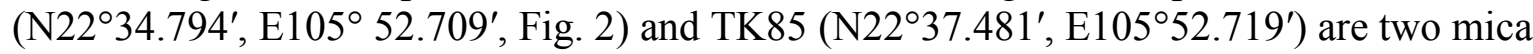
leucogranites from the Pia Oac massif (Fig. 2), which is a small-scale undeformed pluton intruding the Devonian metasedimentary rocks. The granitic pluton is bounded by a normal fault and the surrounding rocks are mylonitic quartzite, micaschist and metapelite. Through microscopic observation (Fig. $4 \mathrm{c}$ and d), the major minerals of the samples are biotite (5$10 \%)$, muscovite (5-10\%), quartz (25-30\%), and feldspar $(<50 \%)$. Sample TK216 (N21 ${ }^{\circ} 50.690^{\prime}, \mathrm{E} 105^{\circ} 00.209^{\prime}$, Fig. 2) is a biotite granite from the Bach Ha pluton (west of Tuyen Quang), which is an undeformed granitic pluton. The magmatic body is located north of the Song Chay suture zone and intrudes into foliated and lineated Devonian marble and paragneiss. Through microscopic observation (Fig. 4e), the major mineral components of the sample are biotite (10-15\%), quartz (25-35\%) and feldspar (<50\%). Sample TK164 (N22 $28.719^{\prime}$, E105 $31.409^{\prime}$, Fig. 2) is a quartz-syenite from the Pia Ma massif (Fig. 2), which forms a crescent-shaped body convex to the east. This foliated and lineated pluton intrudes into the Devonian metapelites and marbles. Through microscopic observation (Fig. 4f), the major constitutive minerals of the sample are biotite $(<5 \%)$, amphibole $(15-20 \%)$, quartz (10-15\%) and feldspar (<60\%). Sample TK264 (N21 $49.302^{\prime}$, E105 $32.848^{\prime}$, Fig. 2) is a granodiorite from the large-scale Diem Mac granitic pluton (SW of Cho Chu, Fig. 2). This pluton, equivalent to the Phia Bioc massif (west of Bac Khan, Fig. 2), intrudes into foliated and weakly metamorphosed Ordovician pelitic rocks, and is covered by Upper Triassic conglomerates. Through the microscopic observation (Fig. 4g), the major mineral components of the sample are amphibole (20-30\%), quartz (20-25\%) and feldspar $(<50 \%)$. Sample TK61 (N22 $34.310^{\prime}, \mathrm{E} 105^{\circ} 38.295^{\prime}$, Fig. 2) is a granodiorite from the Có Linh pluton (Fig. 2), which is a small-scale stock with a weak foliation, intruding into a ductilely deformed but unmetamorphosed Devonian pelite and thin bedded limestone series. Through microscopic observation (Fig. 4h), the major minerals are biotite (15-20\%), quartz (10-15\%), feldspar $(<60 \%)$ and accessory minerals $(<5 \%)$.

\subsection{SIMS U-Pb dating methods}

Zircon concentrates were separated from approximately $2 \mathrm{~kg}$ rock samples by conventional magnetic and density techniques to concentrate non-magnetic, heavy fractions. Zircon grains, together with 91,500, Plešovice, and Penglai zircon standards were mounted in epoxy mounts, which were then polished to section the crystals in half for analysis. All zircon structures were documented with transmitted and reflected light photomicrographs, as well as cathodoluminescence (CL) images, to reveal their internal structures. Each mount was vacuum-coated with high-purity gold prior to secondary ion mass spectrometry (SIMS) analysis.

Measurements of $\mathrm{U}$, Th and $\mathrm{Pb}$ were conducted using the Cameca IMS-1280 SIMS at the Institute of Geology and Geophysics, Chinese Academy of Sciences at Beijing. U-Th- $\mathrm{Pb}$ ratios and absolute abundances were determined relative to the standard zircon Plešovice (Sláma et al., 2008) and 91,500 (Wiedenbeck et al., 1995), analyses of which were interspersed with those of unknown grains, using operating and data processing procedures similar to those described by Li et al. (2009). A long-term uncertainty of 1.5\% (1 RSD) for ${ }^{206} \mathrm{~Pb} /{ }^{238} \mathrm{U}$ measurements of the standard zircons was propagated to the unknowns (Li et al., 
2010b), despite that the measured ${ }^{206} \mathrm{~Pb} /{ }^{238} \mathrm{U}$ error in a specific session is generally around $1 \%$ (1 RSD) or less. Measured compositions were corrected for common $\mathrm{Pb}$ using non-radiogenic ${ }^{204} \mathrm{~Pb}$. Corrections are sufficiently small to be insensitive to the choice of common $\mathrm{Pb}$ composition, and an average of present-day crustal composition (Stacey and Kramers, 1975) is used for the common $\mathrm{Pb}$ assuming that the common $\mathrm{Pb}$ is largely surface contamination introduced during sample preparation. Uncertainties on individual analyses in data tables are reported at a 1sigma level; mean ages for pooled $\mathrm{U} / \mathrm{Pb}($ and $\mathrm{Pb} / \mathrm{Pb}$ ) analyses are quoted with 95\% confidence interval. Data reduction was carried out using the Isoplot/Ex v. 2.49 programs (Ludwig, 2001). SIMS zircon U-Pb isotopic data are presented in Table 2.

Table 2. : SIMS zircon $\mathrm{U}-\mathrm{Pb}$ results. ${ }^{\mathrm{a}}$

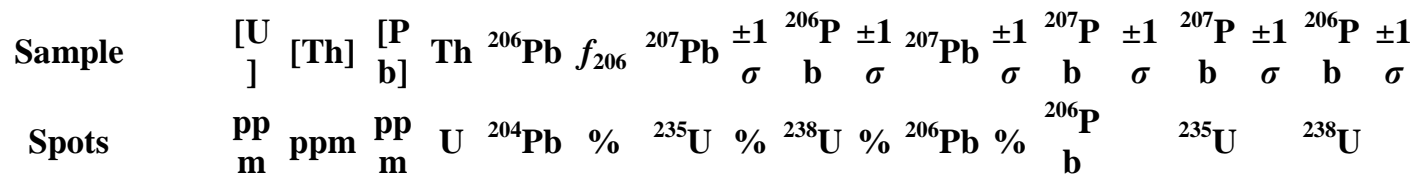

TK61 $\left(22^{\circ} 34.310^{\prime}, 10\right.$ $\left.5^{\circ} 38.295^{\prime}\right)$

TK61@1 $\begin{array}{llllllllllllllll}58 & 4843 & 31 & 0.8 & 1394 & 0.1 & 0.29 & 1 . & 0.04 & 1 . & 0.05 & 0 . & 266.12 . & 259.3 . & 259.3\end{array}$

TK61@2 $\begin{array}{lllllllllllllllll}18 & 1 & 32 & 6 & 3\} & 155 & 60 & 10 & 50 & 156 & 56 & 1 & 8 & 8 & 7 & 1 & 8 \\ 30 & 14 & 14 & 0.5 & 7779 & \{0.0 & 0.28 & 1 . & 0.04 & 1 . & 0.05 & 0 . & 242 . & 16 & 252 . & 3 . & 253 .\end{array}$ $\begin{array}{llllllllllllllllll}01 & 1681 & 7 & 60 & 1 & 2\} & 274 & 66 & 02 & 50 & 104 & 70 & 7 & 1 & 8 & 7 & 9 & 7\end{array}$

TK61@3 $\begin{array}{lllllllllllllllll}98 & 449 & 47 & 0.4 & 1931 & \{0.1 & 0.28 & 1 . & 0.03 & 1 . & 0.05 & 1 . & 279 . & 29 . & 254.4 .251 .3 .\end{array}$

TK61@4 $\begin{array}{lllllllllllllllll}31 & 2401 & 16 & 0.7 & 1016 & 0.0 & 0.28 & 1 . & 0.04 & 1 . & 0.05 & 0 . & 268 . & 15.257 .3 . & 256.3 .\end{array}$

TK61@5

TK61@6

TK61@7

TK61@8

TK61@9

TK61@11*

TK61@10

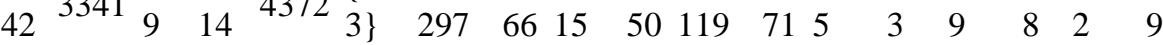
$\begin{array}{lllllllllllllllll}38 & 2554 & 19 & 0.6 & 5021 & \{0.0 & 0.28 & 1 . & 0.04 & 1 . & 0.05 & 0 . & 243 . & 15 . & 257.3 . & 259.3 .\end{array}$

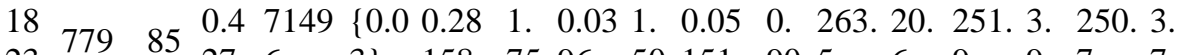
$\begin{array}{lllllllllllllllll}23 & 779 & 85 & 27 & 6 & 3\} & 158 & 75 & 96 & 50 & 151 & 90 & 5 & 6 & 9 & 9 & 7\end{array}$ $\begin{array}{lllllllllllllllll}21 & 948 & 10 & 0.4 & 3274 & 0.0 & 0.27 & 1 . & 0.04 & 1 . & 0.05 & 1.200 .24 .248 .4 .254 . & 2 .\end{array}$

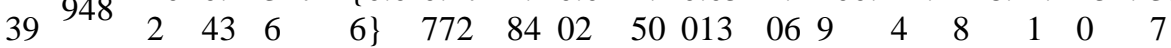
$\begin{array}{llllllllllllllll}28 & 1428 & 13 & 0.5 & 3977 & 0.0 & 0.28 & 1 . & 0.04 & 1 . & 0.05 & 0 . & 241 . & 16.254 .3 . & 256.3 .\end{array}$ $\begin{array}{llllllllllllllll}22 & 1011 & 10 & 0.4 & 9577 & 0.2 & 0.26 & 1 . & 0.03 & 1 . & 0.05 & 0 . & 250 . & 21 . & 238.3 . & 237.3 .\end{array}$

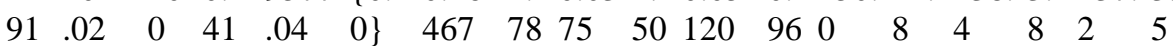
TK61@12 $\begin{array}{lllllllllllllll}15 & 627 & 73 & 0.3 & 2154 & 0.0 & 0.28 & 1.0 .03 & 1 . & 0.05 & 1.251 .26 .251 .4 .251 .3 .\end{array}$ $\begin{array}{llllllllllllllllll}74 & 627 & 73 & 98 & 6 & 9\} & 147 & 89 & 98 & 51 & 123 & 15 & 2 & 2 & 8 & 2 & 9 & 7\end{array}$ $\begin{array}{llllllllllllllllll}29 & 1496 & 14 & 0.5 & 1582 & \{0.0 & 0.29 & 1 . & 0.04 & 1 . & 0.05 & 0 . & 276 & 18 . & 262 . & 4 & 261 . & 3 . \\ 18 & & 6 & 13 & 61 & 1\} & 523 & 71 & 13 & 50 & 180 & 81 & 8 & 5 & 7 & 0 & 1 & 8\end{array}$

TK61@13*

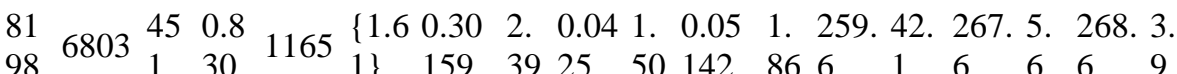

TK61@14

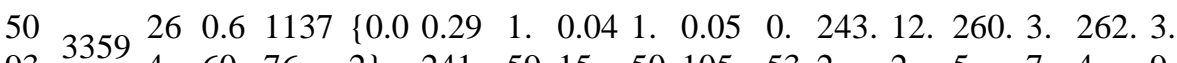
$\begin{array}{llllllllllllllllll}93 & 3359 & 4 & 60 & 76 & 2\} & 241 & 59 & 15 & 50 & 105 & 53 & 2 & 2 & 5 & 7 & 4 & 9\end{array}$

TK61@15*

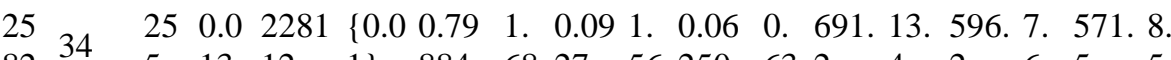
$\begin{array}{llllllllllllllllll}82 & 34 & 5 & 13 & 12 & 1\} & 884 & 68 & 27 & 56 & 250 & 63 & 2 & 4 & 2 & 6 & 5 & 5\end{array}$

TK61@16 $\begin{array}{llllllllllllllll}37 & 2445 & 19 & 0.6 & 8139 & 0.0 & 0.28 & 1 . & 0.04 & 1 . & 0.05 & 0 . & 245 . & 14 & 257.3 . & 259 . \\ 3 .\end{array}$ $\begin{array}{llllllllllllllllll}72 & 2445 & 2 & 48 & 7 & 2\} & 890 & 63 & 10 & 50 & 111 & 63 & 7 & 5 & 7 & 7 & 0 & 8\end{array}$

TK61@17

$\begin{array}{llllllllllllllll}77 & 87 & 34 & 0.1 & 1985 & \{0.0 & 0.28 & 2 . & 0.04 & 1 . & 0.05 & 1.242 .35 .252 .4 .253 . & 24 .\end{array}$ TK61@18 $\begin{array}{lllllllllllllllllll}20 & 831 & 96 & 0.4 & 5050 & \{0.0 & 0.28 & 1 . & 0.04 & 1 . & 0.05 & 0 . & 253 & 21 . & 256 . & 4 & 256 . & 3 . \\ 17 & & & 12 & 5 & 4\} & 723 & 78 & 06 & 50 & 129 & 96 & 9 & 9 & 4 & 0 & 7 & 8\end{array}$ 


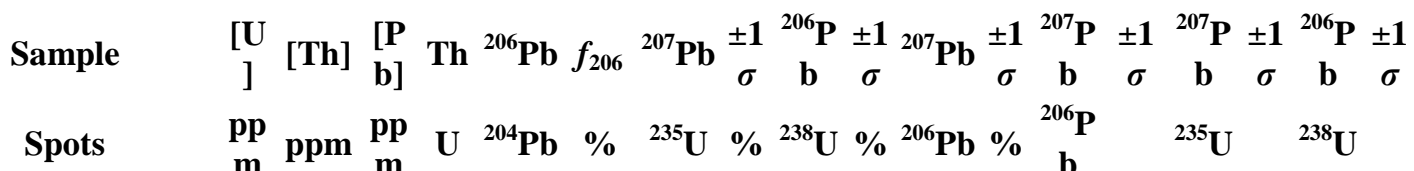

TK61@19

$\begin{array}{lllllllllllllll}22 & 881 & 10 & 0.3 & 1593 & 0.0 & 0.29 & 1 & 0.04 & 1 & 0.05 & 0 . & 283 & 21.260 .4 .257 .3 .\end{array}$

$\begin{array}{llllllllllllllllll}44 & 881 & 7 & 93 & 17 & 1\} & 217 & 77 & 08 & 50 & 196 & 93 & 8 & 2 & 3 & 1 & 7 & 8\end{array}$

TK61@20

$\begin{array}{lllllllllllllllll}39 & 2699 & 20 & 0.6 & 3634 & \{0.0 & 0.29 & 1 . & 0.04 & 1 . & 0.05 & 0 . & 257 . & 15 & 259.3 . & 259.3\end{array}$

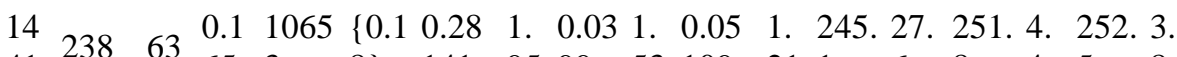

TK61@21

$\begin{array}{llllllllllllllllll}41 & 238 & 63 & 65 & 3 & 8\} & 141 & 95 & 99 & 53 & 109 & 21 & 1 & 6 & 8 & 4 & 5 & 8\end{array}$

TK61@22

$\begin{array}{lllllllllllllllll}36 & 2323 & 18 & 0.6 & 1401 & \{0.0 & 0.29 & 1 . & 0.04 & 1 . & 0.05 & 0 . & 274.14 .262 .3 . & 261.3 . \\ 29 & 232 & 7 & 40 & 58 & 1\} & 515 & 63 & 14 & 50 & 176 & 63 & 8 & 3 & 6 & 8 & 2\end{array}$

$\begin{array}{lllllllllllllllllllll}52 & 3198 & 27 & 0.6 & & \{764 & \{0.2 & 0.29 & 1 & 0.04 & 1 . & 0.05 & 0 . & 252 & 14 & 262 . & 3 & 263 . & 3 . \\ 64 & & 1 & 08 & & 4\} & 521 & 63 & 18 & 50 & 126 & 64 & 6 & 7 & 6 & 8 & 8 & 9\end{array}$

TK61@23

TK84(22 $34.794^{\prime}, 10$

$\left.5^{\circ} 52.709^{\prime}\right)$

TK84@1

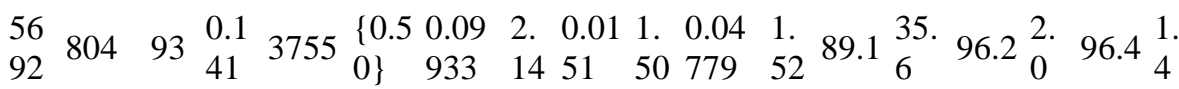

TK84@2

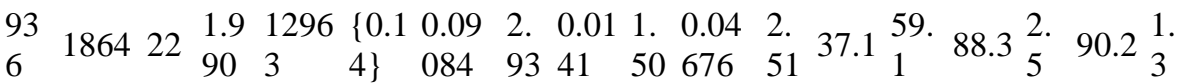

TK84@3

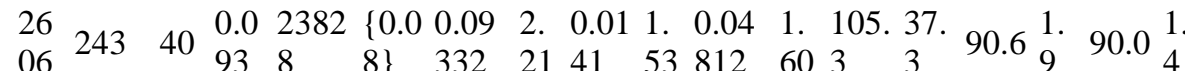

TK84@4

$\begin{array}{llllllllllllllll}11 & 766 & 12 & 0.6 & 3615 & 0.0 & 0.64 & 1 . & 0.08 & 1 . & 0.05 & 0 . & 481 . & 16.507 .6 .513 .7 .\end{array}$

$\begin{array}{llllllllllllllllll}55 & 766 & 0 & 63 & 2 & 5\} & 870 & 68 & 29 & 50 & 674 & 76 & 5 & 8 & 7 & 7 & 5 & 4\end{array}$

TK84@5

$\begin{array}{lllllllllllllllll}34 & 117 & 54 & 0.3 & 8234 & 0.0 & 1.23 & 1 . & 0.13 & 1 . & 0.06 & 0 . & 845.20 .816 .10 & 805.11\end{array}$

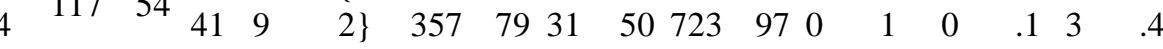

$\begin{array}{llllllllllllllllll}70 & 2327 & 20 & 3.3 & 6308 & \{0.3 & 0.09 & 4 . & 0.01 & 1 . & 0.04 & 4 . & 60.5 & 10 & 89.1 & 4 . & 90.1 & 1 . \\ 2 & & & 14 & & 0\} & 167 & 78 & 41 & 50 & 722 & 54 & & 4.8 & & 1 & & 3\end{array}$

TK84@6

$\begin{array}{llllllllllllllllll}17 & 1235 & 32 & 0.7 & 3587 & \{0.0 & 0.09 & 2 . & 0.01 & 1 . & 0.04 & 1 . & 104 & 40 . & 94.4 & 2 . & 94.0 & 1 . \\ 27 & & & 15 & 6 & 5\} & 740 & 31 & 47 & 53 & 810 & 74 & 0 & 5 & & 1 & & 4\end{array}$

TK84@7

TK84@8

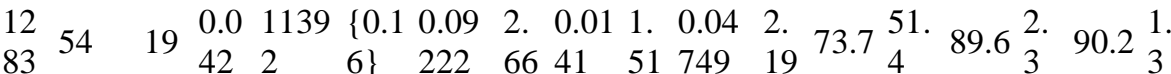

TK84@9

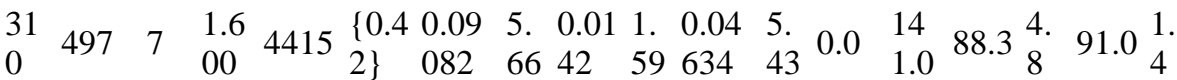

TK84@10

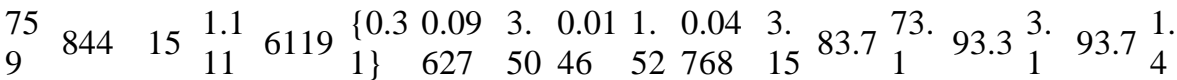

TK84@11

$\begin{array}{llllllllllllllllll}19 & 1052 & 32 & 0.5 & 3142 & \{0.0 & 1.21 & 1 . & 0.13 & 1 & 0.06 & 0 . & 831 & 9.9 & 806.9 & 798 . & 12 \\ 89 & 1052 & 4 & 29 & 0 & 6\} & 365 & 75 & 18 & 68 & 678 & 48 & 0 & 9.9 & 9 & 8 & 2 & .6\end{array}$

TK84@12

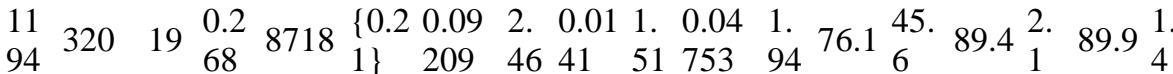

TK84@13

$\begin{array}{llllllllllllllllll}44 & 97 & 70 & 0.2 & 1081 & 0.0 & 1.28 & 1 . & 0.13 & 1 . & 0.06 & 0.850 .17 & 839.10 & 835.11\end{array}$

$\begin{array}{llllllllllllllllll}6 & 97 & 70 & 18 & 24 & 2\} & 638 & 74 & 84 & 51 & 740 & 85 & 2 & 6 & 7 & .0 & 7 & .9\end{array}$

TK84@14

$\begin{array}{lllllllllllllllllllll}19 & 280 & 29 & 0.1 & 2065 & \{0.0 & 0.09 & 2 . & 0.01 & 1 . & 0.04 & 2 . & 40.2 & 53 . & 87.6 & 2 . & 89.3 & 1 . \\ 22 & 280 & 46 & 6 & 9\} & 006 & 74 & 39 & 52 & 682 & 28 & & 7 & & 3 & & 3\end{array}$

TK84@15

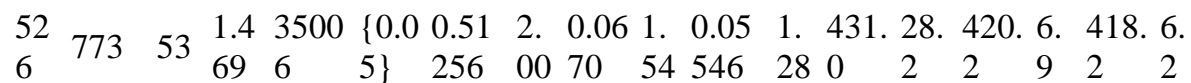

TK $85\left(22^{\circ} 37.481^{\prime}, 10\right.$

$\left.5^{\circ} 52.719^{\prime}\right)$

TK85@1

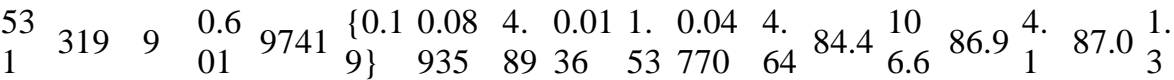

TK85@2

$\begin{array}{lllllllllllllllllll}48 & 354 & 9 & 0.7 & 9439 & \{0.2 & 0.09 & 4 . & 0.01 & 1 . & 0.04 & 3 . & 112 . & 89 . & 91.7 & 3 . & 90.8 & 1 . \\ 8 & & & 25 & & 0\} & 446 & 17 & 42 & 53 & 827 & 88 & 5 & 2 & 91.7 & & & 4\end{array}$

TK85@3

$\begin{array}{llllllllllllllll}41 & 71 & 64 & 0.1 & 3711 & 0.0 & 1.26 & 1 . & 0.13 & 1 . & 0.06 & 1 . & 818.22 .830 .10 & 835.11\end{array}$ 


\begin{tabular}{|c|c|c|c|c|c|c|c|c|c|c|c|c|c|c|c|c|c|c|}
\hline Sample & $\begin{array}{c}{[\mathbf{U}} \\
]\end{array}$ & {$[\mathbf{T h}]$} & {$[\mathbf{P}$} & Th & 206 & $f_{206}$ & ${ }^{207} \mathrm{~Pb}$ & $\begin{array}{c} \pm 1 \\
\sigma\end{array}$ & $\begin{array}{c}{ }^{206} \mathbf{P} \\
\text { b }\end{array}$ & & & \pm 1 & & \pm 1 & b & II & ${ }_{\text {b }}^{206} \mathbf{P}$ & \pm 1 \\
\hline Spots & $\begin{array}{c}\mathbf{p p} \\
\mathbf{m}\end{array}$ & ppm & $\begin{array}{c}\text { pp } \\
\mathbf{m}\end{array}$ & $\mathbf{U}$ & ${ }^{204} \mathrm{~Pb}$ & $\%$ & ${ }^{235} \mathbf{U}$ & $\%$ & ${ }^{238} \mathbf{U}$ & & & & $\begin{array}{l}{ }^{206} \mathbf{P} \\
\mathbf{b}\end{array}$ & & ${ }^{235} \mathbf{U}$ & & ${ }^{238} \mathbf{U}$ & \\
\hline & 3 & & & 73 & 7 & $5\}$ & 608 & 86 & 83 & 51 & 639 & 09 & & 6 & 6 & .6 & 2 & .8 \\
\hline K85@4 & $\begin{array}{l}41 \\
84\end{array}$ & 378 & 64 & $\begin{array}{l}0.0 \\
90\end{array}$ & $\begin{array}{l}1082 \\
08\end{array}$ & $\begin{array}{l}\{0.0 \\
2\}\end{array}$ & $\begin{array}{l}0.09 \\
453\end{array}$ & $\begin{array}{l}1 . \\
80\end{array}$ & $\begin{array}{l}0.01 \\
42\end{array}$ & $\begin{array}{l}1 . \\
51\end{array}$ & $\begin{array}{l}0.04 \\
831\end{array}$ & $\begin{array}{l}0 . \\
99\end{array}$ & $\begin{array}{l}114 . \\
6\end{array}$ & $\begin{array}{l}23 \\
1\end{array}$ & 91.7 & $\begin{array}{l}1 . \\
6\end{array}$ & 90.8 & \\
\hline ГK85@5 & $\begin{array}{l}61 \\
2\end{array}$ & 322 & 10 & $\begin{array}{l}0.5 \\
26\end{array}$ & 6762 & $\begin{array}{l}\{0.2 \\
8\}\end{array}$ & $\begin{array}{l}0.08 \\
974\end{array}$ & $\begin{array}{l}3 . \\
74\end{array}$ & $\begin{array}{l}0.01 \\
38\end{array}$ & $\begin{array}{l}1 . \\
54\end{array}$ & $\begin{array}{l}0.04 \\
701\end{array}$ & $\begin{array}{l}3 . \\
41\end{array}$ & 49.6 & $\begin{array}{l}79 . \\
5\end{array}$ & 87.3 & & 88.6 & \\
\hline K85@6 & $\begin{array}{l}19 \\
92\end{array}$ & 493 & 32 & $\begin{array}{l}0.2 \\
48\end{array}$ & $\begin{array}{l}3856 \\
9\end{array}$ & $\begin{array}{l}\{0.0 \\
5\}\end{array}$ & $\begin{array}{l}0.09 \\
468\end{array}$ & $\begin{array}{l}2 . \\
10\end{array}$ & $\begin{array}{l}0.01 \\
42\end{array}$ & $\begin{array}{l}1 . \\
52\end{array}$ & $\begin{array}{l}0.04 \\
832\end{array}$ & $\begin{array}{l}1 . \\
44\end{array}$ & $\begin{array}{l}115 . \\
0\end{array}$ & $\begin{array}{l}33 . \\
7\end{array}$ & 91.9 & $\begin{array}{l}1 . \\
8\end{array}$ & 91.0 & \\
\hline K85@7 & $\begin{array}{l}51 \\
3\end{array}$ & 695 & 11 & $\begin{array}{l}1.3 \\
56\end{array}$ & 5569 & $\begin{array}{l}\{0.3 \\
4\}\end{array}$ & $\begin{array}{l}0.09 \\
533\end{array}$ & $\begin{array}{l}3 . \\
23\end{array}$ & $\begin{array}{l}0.01 \\
42\end{array}$ & $\begin{array}{l}1 . \\
55\end{array}$ & $\begin{array}{l}0.04 \\
878\end{array}$ & $\begin{array}{l}2 . \\
83\end{array}$ & $\begin{array}{l}137 . \\
1\end{array}$ & $\begin{array}{l}65 \\
2\end{array}$ & 92.5 & $\begin{array}{l}2 . \\
9\end{array}$ & 90.7 & \\
\hline ГK85@8 & $\begin{array}{l}37 \\
79\end{array}$ & 600 & 59 & $\begin{array}{l}0.1 \\
59\end{array}$ & $\begin{array}{l}5194 \\
9\end{array}$ & $\begin{array}{l}\{0.0 \\
4\}\end{array}$ & $\begin{array}{l}0.09 \\
284\end{array}$ & $\begin{array}{l}1 . \\
88\end{array}$ & $\begin{array}{l}0.01 \\
43\end{array}$ & $\begin{array}{l}1 . \\
50\end{array}$ & $\begin{array}{l}0.04 \\
701\end{array}$ & $\begin{array}{l}1 . \\
12\end{array}$ & 49.6 & $\begin{array}{l}26 . \\
5\end{array}$ & 90. & $\begin{array}{l}1 . \\
6\end{array}$ & 91.7 & \\
\hline ГK85@9 & $\begin{array}{l}28 \\
98\end{array}$ & 5388 & 64 & $\begin{array}{l}1.8 \\
59\end{array}$ & 8201 & $\begin{array}{l}\{0.2 \\
3\}\end{array}$ & $\begin{array}{l}0.09 \\
645\end{array}$ & $\begin{array}{l}2 . \\
27\end{array}$ & $\begin{array}{l}0.01 \\
44\end{array}$ & $\begin{array}{l}1 . \\
51\end{array}$ & $\begin{array}{l}0.04 \\
857\end{array}$ & $\begin{array}{l}1 . \\
69\end{array}$ & $\begin{array}{l}127 . \\
1\end{array}$ & $\begin{array}{l}39 \\
3\end{array}$ & 93. & $\begin{array}{l}2 . \\
0\end{array}$ & 92.2 & \\
\hline TK85@10 & $\begin{array}{l}38 \\
23\end{array}$ & 380 & 59 & $\begin{array}{l}0.0 \\
99\end{array}$ & $\begin{array}{l}5136 \\
8\end{array}$ & $\begin{array}{l}\{0.0 \\
4\}\end{array}$ & $\begin{array}{l}0.09 \\
517\end{array}$ & $\begin{array}{l}1 . \\
87\end{array}$ & $\begin{array}{l}0.01 \\
43\end{array}$ & $\begin{array}{l}1 . \\
50\end{array}$ & $\begin{array}{l}0.04 \\
841\end{array}$ & $\begin{array}{l}1 . \\
11\end{array}$ & $\begin{array}{l}119 . \\
5\end{array}$ & $\begin{array}{l}26 . \\
0\end{array}$ & 92.3 & $\begin{array}{l}1 . \\
7\end{array}$ & 91.3 & \\
\hline K85@11 & $\begin{array}{l}17 \\
59\end{array}$ & 230 & 27 & $\begin{array}{l}0.1 \\
31\end{array}$ & $\begin{array}{l}3290 \\
8\end{array}$ & $\begin{array}{l}\{0.0 \\
6\}\end{array}$ & $\begin{array}{l}0.09 \\
423\end{array}$ & $\begin{array}{l}2 . \\
18\end{array}$ & $\begin{array}{l}0.01 \\
43\end{array}$ & $\begin{array}{l}1 . \\
52\end{array}$ & $\begin{array}{l}0.04 \\
793\end{array}$ & $\begin{array}{l}1 . \\
57\end{array}$ & & $\begin{array}{l}36 . \\
8\end{array}$ & 91. & $\begin{array}{l}1 . \\
9\end{array}$ & 91.3 & \\
\hline ГK85@12 & $\begin{array}{l}39 \\
1\end{array}$ & 187 & 7 & $\begin{array}{l}0.4 \\
79\end{array}$ & $\begin{array}{l}1130 \\
7\end{array}$ & $\begin{array}{l}\{0.1 \\
7\}\end{array}$ & $\begin{array}{l}0.09 \\
676\end{array}$ & $\begin{array}{l}3 . \\
74\end{array}$ & $\begin{array}{l}0.01 \\
39\end{array}$ & $\begin{array}{l}1 . \\
79\end{array}$ & $\begin{array}{l}0.05 \\
032\end{array}$ & $\begin{array}{l}3 . \\
28\end{array}$ & $\begin{array}{l}209 . \\
6\end{array}$ & $\begin{array}{l}74 \\
4\end{array}$ & 93.8 & $\begin{array}{l}3 . \\
4\end{array}$ & 89.3 & \\
\hline ГК85@13 & $\begin{array}{l}14 \\
2\end{array}$ & 229 & 3 & $\begin{array}{l}1.6 \\
07\end{array}$ & & & $\begin{array}{l}0.09 \\
049\end{array}$ & $\begin{array}{l}6 . \\
60\end{array}$ & $\begin{array}{l}0.01 \\
43\end{array}$ & & $\begin{array}{l}0.04 \\
583\end{array}$ & $\begin{array}{l}6 . \\
14\end{array}$ & $\begin{array}{l}-11 . \\
4\end{array}$ & $\begin{array}{l}14 \\
2.1\end{array}$ & 88. & $\begin{array}{l}5 . \\
6\end{array}$ & 91.7 & \\
\hline ГK85@14 & $\begin{array}{l}23 \\
11\end{array}$ & 535 & 38 & $\begin{array}{l}0.2 \\
31\end{array}$ & $\begin{array}{l}2579 \\
9\end{array}$ & $\begin{array}{l}\{0.0 \\
7\}\end{array}$ & $\begin{array}{l}0.09 \\
725\end{array}$ & $\begin{array}{l}2 . \\
23\end{array}$ & $\begin{array}{l}0.01 \\
47\end{array}$ & $\begin{array}{l}1 . \\
51\end{array}$ & $\begin{array}{l}0.04 \\
789\end{array}$ & $\begin{array}{l}1 . \\
64\end{array}$ & 93.7 & $\begin{array}{l}38 \\
4\end{array}$ & 94. & $\begin{array}{l}2 . \\
0\end{array}$ & 94.3 & \\
\hline K85@15 & $\begin{array}{l}89 \\
6\end{array}$ & 661 & 16 & $\begin{array}{l}0.7 \\
37\end{array}$ & 9268 & $\begin{array}{l}\{0.2 \\
0\}\end{array}$ & $\begin{array}{l}0.09 \\
037\end{array}$ & $\begin{array}{l}3 . \\
17\end{array}$ & $\begin{array}{l}0.01 \\
41\end{array}$ & $\begin{array}{l}1 . \\
53\end{array}$ & $\begin{array}{l}0.04 \\
660\end{array}$ & $\begin{array}{l}2 . \\
77\end{array}$ & 28.8 & $\begin{array}{l}65 . \\
1\end{array}$ & 87.9 & $\begin{array}{l}2 . \\
7\end{array}$ & 90.0 & \\
\hline ГK85@16 & $\begin{array}{l}17 \\
39\end{array}$ & 404 & 27 & $\begin{array}{l}0.2 \\
33\end{array}$ & 1625 & $\begin{array}{l}\{1.1 \\
5\}\end{array}$ & $\begin{array}{l}0.08 \\
772\end{array}$ & $\begin{array}{l}3 . \\
86\end{array}$ & $\begin{array}{l}0.01 \\
40\end{array}$ & & $\begin{array}{l}0.04 \\
556\end{array}$ & $\begin{array}{l}3 . \\
55\end{array}$ & $\begin{array}{l}-25 \\
8\end{array}$ & $\begin{array}{l}83 . \\
9\end{array}$ & 85 & $\begin{array}{l}3 . \\
2\end{array}$ & 89.4 & \\
\hline [K85@17 & $\begin{array}{l}23 \\
52\end{array}$ & 345 & 37 & $\begin{array}{l}0.1 \\
47\end{array}$ & $\begin{array}{l}1110 \\
1\end{array}$ & $\begin{array}{l}\{0.1 \\
7\}\end{array}$ & $\begin{array}{l}0.09 \\
292\end{array}$ & $\begin{array}{l}2 . \\
20\end{array}$ & $\begin{array}{l}0.01 \\
45\end{array}$ & & $\begin{array}{l}0.04 \\
643\end{array}$ & $\begin{array}{l}1 . \\
61\end{array}$ & 20.2 & $\begin{array}{l}38 \\
2\end{array}$ & 90.2 & 1. & 92.9 & \\
\hline TK85@18 & $\begin{array}{l}41 \\
24\end{array}$ & 471 & 63 & $\begin{array}{l}0.1 \\
14\end{array}$ & $\begin{array}{l}2268 \\
05\end{array}$ & $\begin{array}{l}\{0.0 \\
1\}\end{array}$ & $\begin{array}{l}0.09 \\
468\end{array}$ & $\begin{array}{l}1 . \\
84\end{array}$ & $\begin{array}{l}0.01 \\
42\end{array}$ & $\begin{array}{l}1 . \\
51\end{array}$ & $\begin{array}{l}0.04 \\
839\end{array}$ & $\begin{array}{l}1 . \\
05\end{array}$ & $\begin{array}{l}118 \\
4\end{array}$ & $\begin{array}{l}24 . \\
6\end{array}$ & 91 & $\begin{array}{l}1 . \\
6\end{array}$ & 90.8 & \\
\hline TK85@19 & $\begin{array}{l}33 \\
8\end{array}$ & 814 & 9 & $\begin{array}{l}2.4 \\
10\end{array}$ & $\begin{array}{l}1010 \\
2\end{array}$ & $\begin{array}{l}\{0.1 \\
9\}\end{array}$ & $\begin{array}{l}0.09 \\
198\end{array}$ & $\begin{array}{l}4 . \\
08\end{array}$ & $\begin{array}{l}0.01 \\
43\end{array}$ & & $\begin{array}{l}0.04 \\
652\end{array}$ & $\begin{array}{l}3 . \\
73\end{array}$ & & $\begin{array}{l}87 . \\
0\end{array}$ & 89.3 & $\begin{array}{l}3 . \\
5\end{array}$ & 91.8 & \\
\hline TK85@ & $\begin{array}{l}39 \\
4\end{array}$ & 824 & 9 & $\begin{array}{l}2.0 \\
91\end{array}$ & 29 & $\begin{array}{l}\{0.6 \\
4\}\end{array}$ & $\begin{array}{l}0.09 \\
717\end{array}$ & $\begin{array}{l}3 . \\
64\end{array}$ & $\begin{array}{l}0.01 \\
43\end{array}$ & & $\begin{array}{l}0.04 \\
934\end{array}$ & $\begin{array}{l}3 . \\
30\end{array}$ & 164 & $\begin{array}{l}75 \\
3\end{array}$ & 94.2 & $\begin{array}{l}3 . \\
3\end{array}$ & 91.4 & \\
\hline
\end{tabular}

TK164 $\left(22^{\circ} 28.719^{\prime}, 1\right.$ $05^{\circ} 31.409^{\prime}$ )

TK164@1

$\begin{array}{lllllllllllllllll}75 & 79 & 4 & 1.0 & 1053 & \{0.1 & 0.29 & 4 & 0.03 & 1 . & 0.05 & 4 . & 409.93 .260 .10 & 244.3 .\end{array}$

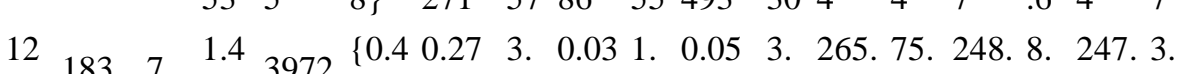

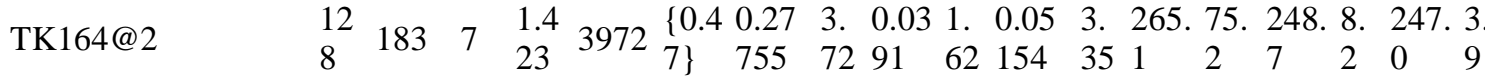
$\begin{array}{llllllllllllllllllll} & & 20 & & & 0.2 & 1206 & \{0.1 & 0.69 & 2 . & 0.08 & 1 & 0.05 & 1 . & 564 & 41 & 533 . & 10 & 526.7 . \\ \text { TK164@3 } & 1 & 47 & 19 & 34 & 8 & 5\} & 145 & 45 & 51 & 50 & 893 & 94 & 7 & 7 & 7 & .2 & 4 & 6\end{array}$

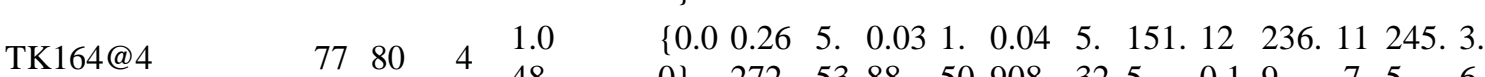

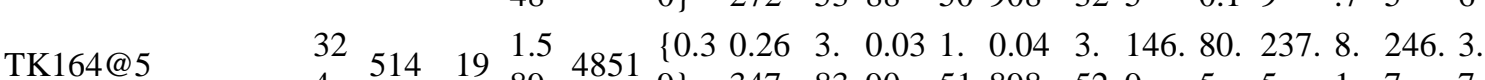
$\begin{array}{llllllllllllllllll}4 & 514 & 19 & 1.5 & 4851 & 9.3 & 347 & 83 & 90 & 51 & 898 & 52 & 9 & 5 & 5 & 1 & 7 & 7\end{array}$ TK164@6 $\begin{array}{llllllllllllllllll}44 & & & 0.7 & 3836 & \{0.0 & 0.67 & 1 . & 0.08 & 1 . & 0.05 & 1 . & 533 & 26 . & 524 & 7 . & 522 . & 7 . \\ 5 & 344 & 49 & 73 & 9 & 5\} & 671 & 92 & 45 & 50 & 810 & 20 & 7 & 1 & 8 & 9 & 7 & 5\end{array}$ 


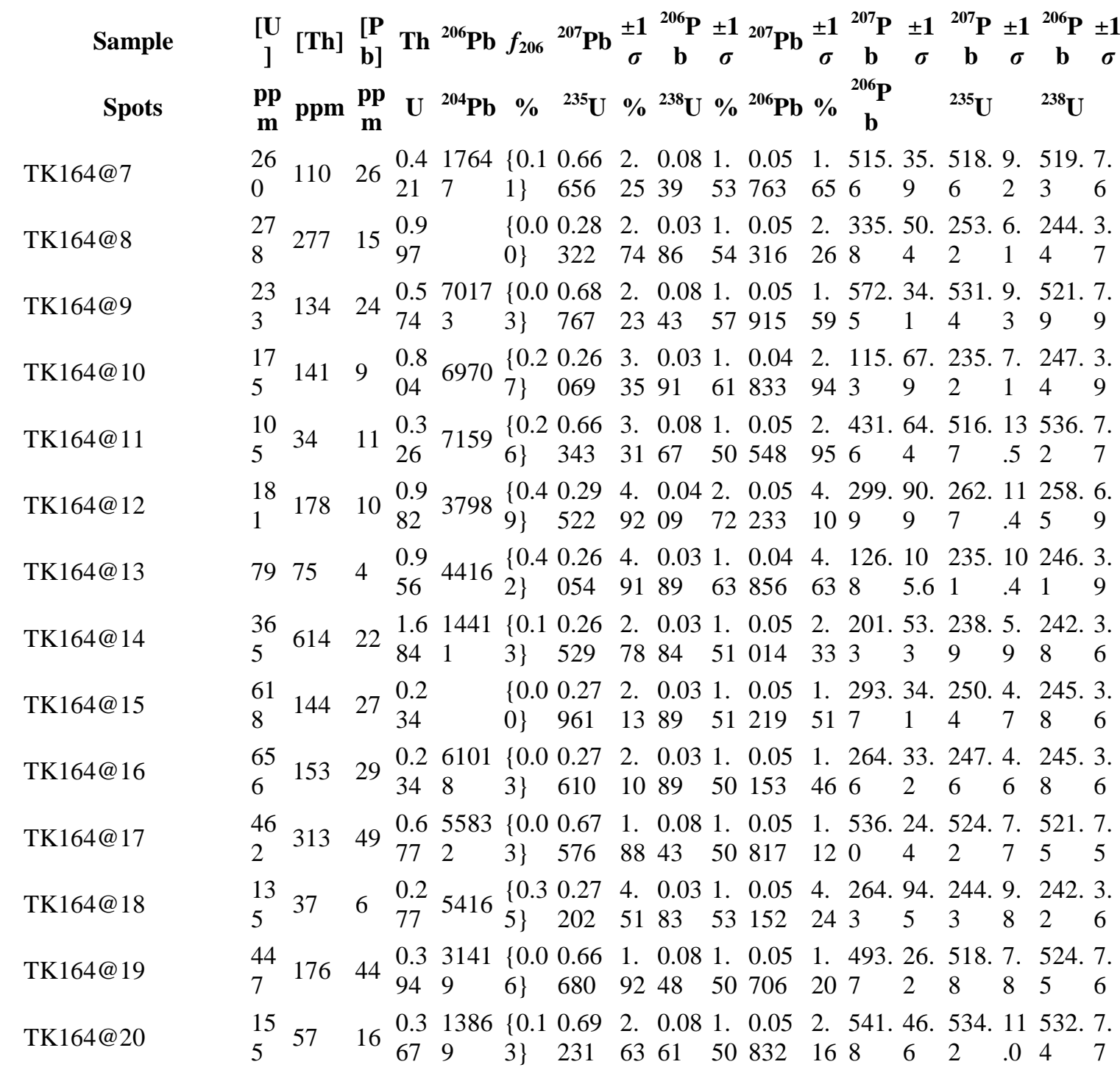

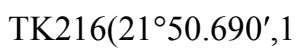
$\left.05^{\circ} 00.209^{\prime}\right)$

TK216@1

$\begin{array}{lllllllllllllllll}15 & 528 & 68 & 0.3 & 3811 & \{0.0 & 0.26 & 1 . & 0.03 & 1 . & 0.05 & 1 . & 199 . & 26.240 .4 .244 .3 .\end{array}$

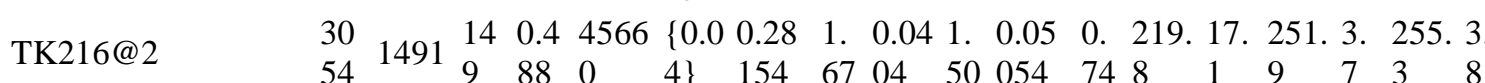

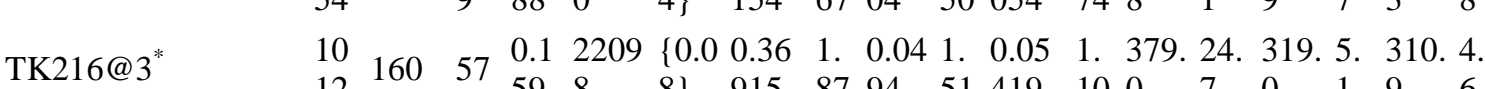

$\begin{array}{lllllllllllllllllllllll}12 & 160 & 57 & 59 & 8 & 8\} & 915 & 87 & 94 & 51 & 419 & 10 & 0 & 7 & 0 & 1 & 9 & 6\end{array}$

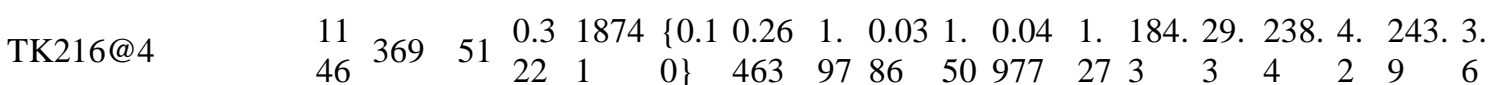

TK216@5* $\quad 92 \begin{array}{lllllllllllllllllll} & & 24 & 0.2 & 2101 & 0.0 & 2.85 & 2 . & 0.22 & 1 & 0.09 & 0 . & 142 & 17 & 137 & 15 & 133 & 21\end{array}$

$\begin{array}{llllllllllllllllll}9 & 205 & 7 & 21 & 69 & 1\} & 584 & 00 & 99 & 77 & 008 & 94 & 7.3 & 9 & 0.3 & .2 & 4.1 & .3\end{array}$

$\begin{array}{lllllllllllllllllllllll} & \text { TK216@6* } & 31 & 112 & 53 & 0.3 & 2193 & \{0.0 & 1.38 & 1 & 0.14 & 1 & 0.07 & 1 . & 937 & 23 & 882 & 11 & 860 & 12 \\ & 4 & & & & 56 & 0 & 9\} & 483 & 89 & 29 & 51 & 031 & 14 & 4 & 1 & 5 & .2 & 8 & .1\end{array}$

$\begin{array}{lllllllllllllllllll} & \text { TK216@7* } & 39 & & 19 & 0.1 & 5644 & \{0.0 & 0.33 & 1 . & 0.04 & 1 . & 0.05 & 0 . & 295 & 13 & 291 . & 4 & 291 . \\ & 45 & 394 & 9 & 00 & 3 & 3\} & 261 & 62 & 62 & 51 & 222 & 59 & 2 & 3 & 6 & 1 & 1 & 3\end{array}$

$\begin{array}{lllllllllllllllll}29 & 615 & 13 & 0.2 & 4567 & 0.0 & 0.27 & 1 . & 0.04 & 1 . & 0.05 & 0 . & 213.16 .250 .3 . & 254.3 .\end{array}$

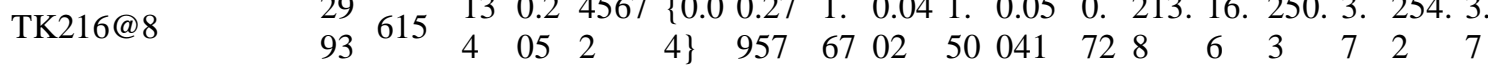

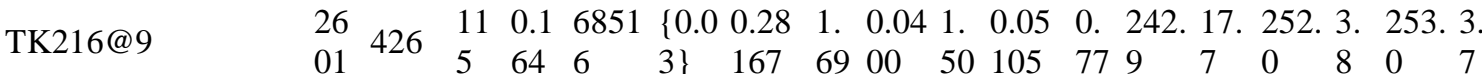

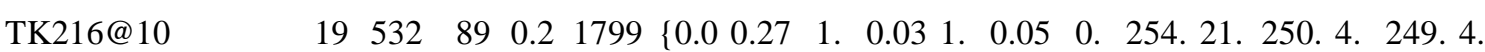




\begin{tabular}{|c|c|c|c|c|c|c|c|c|c|c|c|c|c|c|c|c|c|c|}
\hline Sample & & {$[\mathrm{Th}]$} & $\begin{array}{l}{[\mathbf{P}} \\
\mathbf{b}]\end{array}$ & & ${ }^{206} \mathrm{~Pb}$ & $f_{206}$ & ${ }^{207} \mathrm{~Pb}$ & \pm 1 & & & ${ }^{207} \mathbf{P l}$ & & ${ }_{b}^{07} \mathbf{P}$ & $\begin{array}{c} \pm 1 \\
\sigma\end{array}$ & b & $\begin{array}{c} \pm 1 \\
\sigma\end{array}$ & $\begin{array}{l}{ }^{06} \mathbf{P} \\
\mathrm{b}\end{array}$ & \pm 1 \\
\hline Spots & & ppm & $\begin{array}{c}\text { pp } \\
\text { m }\end{array}$ & $\mathbf{U}$ & ${ }^{204} \mathrm{~Pb}$ & $\%$ & ${ }^{235} \mathbf{U}$ & $\%$ & ${ }^{238} \mathbf{U}$ & $\%$ & Pb & $\%$ & $\begin{array}{l}{ }^{06} \mathbf{P} \\
b\end{array}$ & & ${ }^{235} \mathbf{U}$ & & ${ }^{238} \mathbf{U}$ & \\
\hline & 83 & & & 68 & 04 & $1\}$ & 922 & 97 & 95 & 73 & 130 & 94 & & 6 & 0 & 4 & 6 & 2 \\
\hline 216@ & $\begin{array}{l}18 \\
21\end{array}$ & 73 & $\begin{array}{l}21 \\
4\end{array}$ & $\begin{array}{l}0.0 \\
40\end{array}$ & $\begin{array}{l}1160 \\
51\end{array}$ & $\begin{array}{l}\{0.0 \\
2\}\end{array}$ & $\begin{array}{l}1.01 \\
217\end{array}$ & $\begin{array}{l}1 . \\
61\end{array}$ & $\begin{array}{l}0.10 \\
96\end{array}$ & $\begin{array}{l}1 . \\
54\end{array}$ & $\begin{array}{l}0.06 \\
695\end{array}$ & $\begin{array}{l}0 . \\
49\end{array}$ & $\begin{array}{l}836 . \\
3\end{array}$ & $\begin{array}{l}10 . \\
1\end{array}$ & $\begin{array}{l}710 . \\
0\end{array}$ & $\begin{array}{l}8 . \\
3\end{array}$ & $\begin{array}{l}670 . \\
7\end{array}$ & 8 \\
\hline K216@12 & $\begin{array}{l}26 \\
55\end{array}$ & 294 & $\begin{array}{l}11 \\
7\end{array}$ & $\begin{array}{l}0.1 \\
11\end{array}$ & $\begin{array}{l}3724 \\
8\end{array}$ & $\begin{array}{l}\{0.0 \\
5\}\end{array}$ & $\begin{array}{l}0.28 \\
025\end{array}$ & $\begin{array}{l}1 . \\
69\end{array}$ & $\begin{array}{l}0.04 \\
06\end{array}$ & $\begin{array}{l}1 . \\
50\end{array}$ & $\begin{array}{l}0.05 \\
011\end{array}$ & $\begin{array}{l}0 . \\
77\end{array}$ & $\begin{array}{l}199 . \\
9\end{array}$ & $\begin{array}{l}17 . \\
8\end{array}$ & $\begin{array}{l}250 . \\
9\end{array}$ & $\begin{array}{l}3 . \\
8\end{array}$ & $\begin{array}{l}256 . \\
3\end{array}$ & $\begin{array}{l}3 \\
8\end{array}$ \\
\hline ГK216@13 & $\begin{array}{l}23 \\
47\end{array}$ & 609 & $\begin{array}{l}10 \\
4\end{array}$ & $\begin{array}{l}0.2 \\
59\end{array}$ & 4177 & $\begin{array}{l}\{0.4 \\
5\}\end{array}$ & $\begin{array}{l}0.27 \\
893\end{array}$ & $\begin{array}{l}1 . \\
89\end{array}$ & $\begin{array}{l}0.03 \\
94\end{array}$ & $\begin{array}{l}1 . \\
50\end{array}$ & $\begin{array}{l}0.05 \\
140\end{array}$ & $\begin{array}{l}1 . \\
15\end{array}$ & $\begin{array}{l}259 . \\
0\end{array}$ & $\begin{array}{l}26 . \\
2\end{array}$ & $\begin{array}{l}249 . \\
8\end{array}$ & $\begin{array}{l}4 . \\
2\end{array}$ & $\begin{array}{l}248 . \\
8\end{array}$ & 7 \\
\hline TK216@14* & $\begin{array}{l}30 \\
63\end{array}$ & 402 & $\begin{array}{l}14 \\
3\end{array}$ & $\begin{array}{l}0.1 \\
31\end{array}$ & $\begin{array}{l}1545 \\
38\end{array}$ & $\begin{array}{l}\{0.0 \\
1\}\end{array}$ & $\begin{array}{l}0.30 \\
767\end{array}$ & $\begin{array}{l}1 . \\
64\end{array}$ & $\begin{array}{l}0.04 \\
28\end{array}$ & $\begin{array}{l}1 . \\
50\end{array}$ & $\begin{array}{l}0.05 \\
208\end{array}$ & $\begin{array}{l}0 . \\
65\end{array}$ & $\begin{array}{l}288 . \\
9\end{array}$ & $\begin{array}{l}14 . \\
8\end{array}$ & $\begin{array}{l}272 . \\
4\end{array}$ & $\begin{array}{l}3 . \\
9\end{array}$ & $\begin{array}{l}270 . \\
5\end{array}$ & $\begin{array}{l}4 . \\
0\end{array}$ \\
\hline 'K216@15 & $\begin{array}{l}43 \\
46\end{array}$ & 458 & $\begin{array}{l}19 \\
5\end{array}$ & $\begin{array}{l}0.1 \\
05\end{array}$ & $\begin{array}{l}6901 \\
0\end{array}$ & $\begin{array}{l}\{0.0 \\
3\}\end{array}$ & $\begin{array}{l}0.29 \\
260\end{array}$ & $\begin{array}{l}1 . \\
61\end{array}$ & $\begin{array}{l}0.04 \\
15\end{array}$ & $\begin{array}{l}1 . \\
50\end{array}$ & $\begin{array}{l}0.05 \\
118\end{array}$ & $\begin{array}{l}0 . \\
59\end{array}$ & $\begin{array}{l}248 . \\
9\end{array}$ & $\begin{array}{l}13 . \\
4\end{array}$ & $\begin{array}{l}260 . \\
6\end{array}$ & $\begin{array}{l}3 . \\
7\end{array}$ & $\begin{array}{l}261 . \\
9\end{array}$ & $\begin{array}{l}3 . \\
9\end{array}$ \\
\hline K216@16 & $\begin{array}{l}23 \\
76\end{array}$ & 380 & $\begin{array}{l}10 \\
5\end{array}$ & $\begin{array}{l}0.1 \\
60\end{array}$ & $\begin{array}{l}3714 \\
3\end{array}$ & $\begin{array}{l}\{0.0 \\
5\}\end{array}$ & $\begin{array}{l}0.27 \\
863\end{array}$ & $\begin{array}{l}1 . \\
73\end{array}$ & $\begin{array}{l}0.04 \\
03\end{array}$ & $\begin{array}{l}1 . \\
50\end{array}$ & $\begin{array}{l}0.05 \\
014\end{array}$ & $\begin{array}{l}0 . \\
86\end{array}$ & & $\begin{array}{l}19 . \\
9\end{array}$ & $\begin{array}{l}249 . \\
6\end{array}$ & $\begin{array}{l}3 . \\
8\end{array}$ & & 3. \\
\hline ГK216@17 & $\begin{array}{l}22 \\
83\end{array}$ & 960 & $\begin{array}{l}10 \\
7\end{array}$ & $\begin{array}{l}0.4 \\
21\end{array}$ & $\begin{array}{l}3929 \\
4\end{array}$ & $\begin{array}{l}\{0.0 \\
5\}\end{array}$ & $\begin{array}{l}0.28 \\
023\end{array}$ & $\begin{array}{l}1 . \\
71\end{array}$ & $\begin{array}{l}0.03 \\
99\end{array}$ & $\begin{array}{l}1 . \\
50\end{array}$ & $\begin{array}{l}0.05 \\
097\end{array}$ & $\begin{array}{l}0 . \\
83\end{array}$ & & $\begin{array}{l}19 . \\
0\end{array}$ & $\begin{array}{l}250 . \\
8\end{array}$ & $\begin{array}{l}3 . \\
8\end{array}$ & & \\
\hline ГK216@18 & & 589 & $\begin{array}{l}15 \\
7\end{array}$ & $\begin{array}{l}0.1 \\
69\end{array}$ & $\begin{array}{l}9025 \\
5\end{array}$ & $\begin{array}{l}\{0.0 \\
2\}\end{array}$ & $\begin{array}{l}0.28 \\
676\end{array}$ & $\begin{array}{l}1 . \\
64\end{array}$ & $\begin{array}{l}0.04 \\
10\end{array}$ & $\begin{array}{l}1 . \\
50\end{array}$ & $\begin{array}{l}0.05 \\
078\end{array}$ & $\begin{array}{l}0 . \\
66\end{array}$ & $\begin{array}{l}230 . \\
7\end{array}$ & $\begin{array}{l}15 . \\
1\end{array}$ & $\begin{array}{l}256 . \\
0\end{array}$ & $\begin{array}{l}3 . \\
7\end{array}$ & & $\begin{array}{l}3 . \\
8\end{array}$ \\
\hline ГК216@19 & & 401 & 76 & $\begin{array}{l}0.2 \\
39\end{array}$ & $\begin{array}{l}4613 \\
7\end{array}$ & $\begin{array}{l}\{0.0 \\
4\}\end{array}$ & $\begin{array}{l}0.28 \\
195\end{array}$ & $\begin{array}{l}1 . \\
85\end{array}$ & $\begin{array}{l}0.04 \\
02\end{array}$ & $\begin{array}{l}1 . \\
50\end{array}$ & $\begin{array}{l}0.05 \\
092\end{array}$ & $\begin{array}{l}1 . \\
08\end{array}$ & $\begin{array}{l}237 . \\
1\end{array}$ & $\begin{array}{l}24 . \\
8\end{array}$ & $\begin{array}{l}252 . \\
2\end{array}$ & $\begin{array}{l}4 . \\
1\end{array}$ & $\begin{array}{l}253 . \\
8\end{array}$ & $\begin{array}{l}3 . \\
7\end{array}$ \\
\hline ГK216@20 & & 1211 & $\begin{array}{l}13 \\
5\end{array}$ & $\begin{array}{l}0.4 \\
29\end{array}$ & $\begin{array}{l}4353 \\
3\end{array}$ & $\begin{array}{l}\{0.0 \\
4\}\end{array}$ & $\begin{array}{l}0.28 \\
205\end{array}$ & $\begin{array}{l}1 . \\
82\end{array}$ & $\begin{array}{l}0.04 \\
05\end{array}$ & $\begin{array}{l}1 . \\
50\end{array}$ & $\begin{array}{l}0.05 \\
050\end{array}$ & $\begin{array}{l}1 . \\
03\end{array}$ & $\begin{array}{l}218 . \\
0\end{array}$ & $\begin{array}{l}23 . \\
7\end{array}$ & $\begin{array}{l}252 . \\
3\end{array}$ & $\begin{array}{l}4 . \\
1\end{array}$ & $\begin{array}{l}256 . \\
0\end{array}$ & 3. \\
\hline K216C & & 2158 & $\begin{array}{l}20 \\
1\end{array}$ & $\begin{array}{l}0.5 \\
23\end{array}$ & $\begin{array}{l}3385 \\
8\end{array}$ & $\begin{array}{l}\{0.0 \\
6\}\end{array}$ & $\begin{array}{l}0.28 \\
074\end{array}$ & $\begin{array}{l}1 . \\
67\end{array}$ & $\begin{array}{l}0.04 \\
01\end{array}$ & 1. & $\begin{array}{l}0.05 \\
076\end{array}$ & $\begin{array}{l}0 . \\
72\end{array}$ & $\begin{array}{l}229 . \\
9\end{array}$ & $\begin{array}{l}16 . \\
5\end{array}$ & $\begin{array}{l}251 . \\
2\end{array}$ & $\begin{array}{l}3 . \\
7\end{array}$ & $\begin{array}{l}253 . \\
5\end{array}$ & 3. \\
\hline \multicolumn{19}{|c|}{$\begin{array}{l}\text { TK264(21 }{ }^{\circ} 49.302^{\prime}, 1 \\
\left.05^{\circ} 32.848^{\prime}\right)\end{array}$} \\
\hline ГК264@1* & 49 & 152 & 76 & $\begin{array}{l}0.3 \\
10\end{array}$ & $\begin{array}{l}6251 \\
5\end{array}$ & $\begin{array}{l}\{0.0 \\
3\}\end{array}$ & $\begin{array}{l}1.48 \\
097\end{array}$ & $\begin{array}{l}1 . \\
84\end{array}$ & $\begin{array}{l}0.12 \\
76\end{array}$ & $\begin{array}{l}1 . \\
68\end{array}$ & $\begin{array}{l}0.08 \\
415\end{array}$ & $\begin{array}{l}0 . \\
76\end{array}$ & $\begin{array}{l}129 \\
6.1\end{array}$ & $\begin{array}{l}14 . \\
8\end{array}$ & $\begin{array}{l}922 . \\
6\end{array}$ & $\begin{array}{l}11 \\
.2\end{array}$ & $\begin{array}{l}774 . \\
4\end{array}$ & $\begin{array}{l}12 \\
.3\end{array}$ \\
\hline K264@2 & & 165 & 34 & $\begin{array}{l}0.2 \\
19\end{array}$ & & & & $\begin{array}{l}2 . \\
48\end{array}$ & $\begin{array}{l}0.04 \\
02\end{array}$ & $\begin{array}{l}1 . \\
50\end{array}$ & & $\begin{array}{l}1 . \\
98\end{array}$ & $\begin{array}{l}308 . \\
5\end{array}$ & $\begin{array}{l}44 . \\
4\end{array}$ & $\begin{array}{l}259 . \\
5\end{array}$ & $\begin{array}{l}5 . \\
7\end{array}$ & $\begin{array}{l}254 . \\
1\end{array}$ & \\
\hline 'K264@ & 59 & 252 & 27 & $\begin{array}{l}0.4 \\
26\end{array}$ & $\begin{array}{l}3191 \\
1\end{array}$ & $\begin{array}{l}\{0.0 \\
6\}\end{array}$ & $\begin{array}{l}0.27 \\
603\end{array}$ & $\begin{array}{l}2 . \\
15\end{array}$ & $\begin{array}{l}0.03 \\
91\end{array}$ & $\begin{array}{l}1 . \\
50\end{array}$ & & $\begin{array}{l}1 . \\
54\end{array}$ & & $\begin{array}{l}35 . \\
1\end{array}$ & $\begin{array}{l}247 . \\
5\end{array}$ & $\begin{array}{l}4 . \\
7\end{array}$ & & 3. \\
\hline K264@4 & 4 & 215 & 24 & $\begin{array}{l}0.4 \\
17\end{array}$ & $\begin{array}{l}1997 \\
3\end{array}$ & $\begin{array}{l}\{0.0 \\
9\}\end{array}$ & $\begin{array}{l}0.27 \\
671\end{array}$ & $\begin{array}{l}2 . \\
26\end{array}$ & $\begin{array}{l}0.03 \\
98\end{array}$ & $\begin{array}{l}1 . \\
52\end{array}$ & $\begin{array}{l}0.05 \\
048\end{array}$ & $\begin{array}{l}1 . \\
67\end{array}$ & $\begin{array}{l}217 . \\
2\end{array}$ & $\begin{array}{l}38 \\
2\end{array}$ & $\begin{array}{l}248 . \\
0\end{array}$ & $\begin{array}{l}5 . \\
0\end{array}$ & $\begin{array}{l}251 . \\
3\end{array}$ & $\begin{array}{l}3 \\
7\end{array}$ \\
\hline K264C & 75 & 158 & 33 & $\begin{array}{l}0.2 \\
11\end{array}$ & $\begin{array}{l}1941 \\
5\end{array}$ & $\begin{array}{l}\{0.1 \\
0\}\end{array}$ & $\begin{array}{l}0.27 \\
877\end{array}$ & $\begin{array}{l}2 . \\
03\end{array}$ & $\begin{array}{l}0.03 \\
98\end{array}$ & $\begin{array}{l}1 . \\
50\end{array}$ & $\begin{array}{l}0.05 \\
080\end{array}$ & $\begin{array}{l}1 . \\
37\end{array}$ & $\begin{array}{l}231 . \\
7\end{array}$ & $\begin{array}{l}31 . \\
4\end{array}$ & $\begin{array}{l}249 . \\
7\end{array}$ & $\begin{array}{l}4 . \\
5\end{array}$ & $\begin{array}{l}251 . \\
6\end{array}$ & $\begin{array}{l}3 . \\
7\end{array}$ \\
\hline ГK264@6 & & 325 & 29 & $\begin{array}{l}0.5 \\
44\end{array}$ & $\begin{array}{l}5220 \\
1\end{array}$ & $\begin{array}{l}\{0.0 \\
4\}\end{array}$ & $\begin{array}{l}0.28 \\
279\end{array}$ & $\begin{array}{l}2 . \\
41\end{array}$ & $\begin{array}{l}0.03 \\
95\end{array}$ & $\begin{array}{l}1 . \\
51\end{array}$ & & $\begin{array}{l}1 . \\
88\end{array}$ & 281. & $\begin{array}{l}42 . \\
4\end{array}$ & $\begin{array}{l}252 . \\
9\end{array}$ & $\begin{array}{l}5 \\
4\end{array}$ & & $\begin{array}{l}3 \\
7\end{array}$ \\
\hline TK264@7* & & 292 & 39 & $\begin{array}{l}0.3 \\
54\end{array}$ & & $\begin{array}{l}\{1.0 \\
3\}\end{array}$ & $\begin{array}{l}0.28 \\
466\end{array}$ & $\begin{array}{l}9 . \\
32\end{array}$ & $\begin{array}{l}0.04 \\
04\end{array}$ & 1. & $\begin{array}{l}0.05 \\
106\end{array}$ & $\begin{array}{l}9 . \\
20\end{array}$ & $\begin{array}{l}243 . \\
7\end{array}$ & $\begin{array}{l}19 \\
9.1\end{array}$ & $\begin{array}{l}254 . \\
3\end{array}$ & $\begin{array}{l}21 \\
.2\end{array}$ & $\begin{array}{l}255 . \\
5\end{array}$ & $\begin{array}{l}3 \\
8\end{array}$ \\
\hline TK264@8* & $\begin{array}{l}59 \\
4\end{array}$ & 378 & $\begin{array}{l}11 \\
7\end{array}$ & $\begin{array}{l}0.6 \\
37\end{array}$ & $\begin{array}{l}9145 \\
8\end{array}$ & $\begin{array}{l}\{0.0 \\
2\}\end{array}$ & $\begin{array}{l}1.60 \\
039\end{array}$ & $\begin{array}{l}1 . \\
66\end{array}$ & $\begin{array}{l}0.15 \\
52\end{array}$ & $\begin{array}{l}1 . \\
50\end{array}$ & $\begin{array}{l}0.07 \\
478\end{array}$ & $\begin{array}{l}0 . \\
70\end{array}$ & $\begin{array}{l}106 \\
2.7\end{array}$ & $\begin{array}{l}14 . \\
0\end{array}$ & $\begin{array}{l}970 . \\
4\end{array}$ & $\begin{array}{l}10 \\
.4\end{array}$ & $\begin{array}{l}930 . \\
1\end{array}$ & \\
\hline TK264@9* & $\begin{array}{l}43 \\
5\end{array}$ & 179 & 20 & $\begin{array}{l}0.4 \\
10\end{array}$ & 1228 & $\begin{array}{l}\{1.5 \\
2\}\end{array}$ & $\begin{array}{l}0.27 \\
704\end{array}$ & $\begin{array}{l}7 . \\
22\end{array}$ & $\begin{array}{l}0.03 \\
89\end{array}$ & $\begin{array}{l}1 . \\
52\end{array}$ & $\begin{array}{l}0.05 \\
169\end{array}$ & $\begin{array}{l}7 . \\
05\end{array}$ & $\begin{array}{l}271 . \\
8\end{array}$ & $\begin{array}{l}15 \\
4.1\end{array}$ & $\begin{array}{l}248 . \\
3\end{array}$ & $\begin{array}{l}16 \\
.0\end{array}$ & $\begin{array}{l}245 . \\
8\end{array}$ & .3 \\
\hline ГK264@10 & 41 & 153 & 19 & $\begin{array}{l}0.3 \\
67\end{array}$ & & $\begin{array}{l}\{0.0 \\
0\}\end{array}$ & $\begin{array}{l}0.28 \\
697\end{array}$ & $\begin{array}{l}2 . \\
71\end{array}$ & $\begin{array}{l}0.04 \\
01\end{array}$ & 1. & $\begin{array}{l}0.05 \\
196\end{array}$ & $\begin{array}{l}2 . \\
25\end{array}$ & $\begin{array}{l}283 . \\
8\end{array}$ & $\begin{array}{l}50 . \\
7\end{array}$ & $\begin{array}{l}256 . \\
2\end{array}$ & $\begin{array}{l}6 \\
2\end{array}$ & $\begin{array}{l}253 . \\
2\end{array}$ & $\begin{array}{l}3 \\
7\end{array}$ \\
\hline TK264@11* & 12 & 262 & 57 & $\begin{array}{l}0.2 \\
12\end{array}$ & & $\begin{array}{l}\{0.0 \\
0\}\end{array}$ & $\begin{array}{l}0.28 \\
908\end{array}$ & $\begin{array}{l}1 . \\
83\end{array}$ & $\begin{array}{l}0.04 \\
09\end{array}$ & 1. & $\begin{array}{l}0.05 \\
127\end{array}$ & $\begin{array}{l}1 . \\
05\end{array}$ & $\begin{array}{l}253 . \\
0\end{array}$ & $\begin{array}{l}23 . \\
9\end{array}$ & $\begin{array}{l}257 . \\
8\end{array}$ & $\begin{array}{l}4 . \\
2\end{array}$ & $\begin{array}{l}258 . \\
4\end{array}$ & .3 \\
\hline K264@12 & $\begin{array}{l}39 \\
6\end{array}$ & 174 & 19 & $\begin{array}{l}0.4 \\
40\end{array}$ & $\begin{array}{l}1249 \\
0\end{array}$ & $\begin{array}{l}\{0.1 \\
5\}\end{array}$ & $\begin{array}{l}0.28 \\
154\end{array}$ & $\begin{array}{l}2 . \\
48\end{array}$ & $\begin{array}{l}0.03 \\
99\end{array}$ & $\begin{array}{l}1 . \\
51\end{array}$ & $\begin{array}{l}0.05 \\
123\end{array}$ & $\begin{array}{l}1 . \\
97\end{array}$ & $\begin{array}{l}251 . \\
1\end{array}$ & $\begin{array}{l}44 \\
6\end{array}$ & $\begin{array}{l}251 . \\
9\end{array}$ & $\begin{array}{l}5 . \\
5\end{array}$ & $\begin{array}{l}252 . \\
0\end{array}$ & .3 \\
\hline
\end{tabular}




\begin{tabular}{|c|c|c|c|c|c|c|c|c|c|c|c|c|c|c|c|c|c|c|}
\hline Sample & J & {$[\mathrm{Th}]$} & {$[\mathbf{P}$} & Th & ${ }^{206} \mathrm{~Pb}$ & $f_{206}$ & ${ }^{207} \mathbf{P b}$ & \pm 1 & $\begin{array}{c}{ }^{206} \mathbf{P} \\
\mathrm{b}\end{array}$ & & ${ }^{07} \mathrm{~Pb}$ & $\begin{array}{c} \pm 1 \\
\sigma\end{array}$ & $\begin{array}{c}{ }^{207} \mathbf{P} \\
\mathbf{b}\end{array}$ & $\begin{array}{c} \pm 1 \\
\sigma\end{array}$ & b & $\begin{array}{c} \pm 1 \\
\sigma\end{array}$ & ${ }_{b}^{006} \mathbf{P}$ & \\
\hline Spots & & ppm & $\begin{array}{c}\text { pp } \\
\text { m }\end{array}$ & $\mathbf{U}$ & ${ }^{204} \mathrm{~Pb}$ & $\%$ & ${ }^{235} \mathbf{U}$ & $\%$ & ${ }^{238} \mathbf{U}$ & & ${ }^{206} \mathrm{~Pb}$ & $\%$ & $\begin{array}{c}{ }^{206} \mathbf{P} \\
\text { b }\end{array}$ & & ${ }^{235} \mathbf{U}$ & & ${ }^{238} \mathbf{U}$ & \\
\hline K264@13 & $\begin{array}{l}12 \\
37\end{array}$ & 248 & 55 & $\begin{array}{l}0.2 \\
00\end{array}$ & $\begin{array}{l}7236 \\
0\end{array}$ & $\begin{array}{l}\{0.0 \\
3\}\end{array}$ & $\begin{array}{l}0.28 \\
749\end{array}$ & $\begin{array}{l}1 . \\
84\end{array}$ & $\begin{array}{l}0.04 \\
01\end{array}$ & $\begin{array}{l}1 . \\
50\end{array}$ & $\begin{array}{l}0.05 \\
202\end{array}$ & $\begin{array}{l}1.2 \\
05\end{array}$ & $\begin{array}{l}286 . \\
1\end{array}$ & $\begin{array}{l}23 \\
9\end{array}$ & & $\begin{array}{l}4 . \\
2\end{array}$ & & t \\
\hline K264@14 & 71 & 142 & 32 & $\begin{array}{l}0.1 \\
98\end{array}$ & $\begin{array}{l}2316 \\
8\end{array}$ & $\begin{array}{l}\{0.0 \\
8\}\end{array}$ & $\begin{array}{l}0.28 \\
113\end{array}$ & $\begin{array}{l}2 . \\
47\end{array}$ & $\begin{array}{l}0.04 \\
03\end{array}$ & $\begin{array}{l}1 . \\
50\end{array}$ & $\begin{array}{l}0.05 \\
055\end{array}$ & $\begin{array}{l}1.2 \\
963\end{array}$ & $\begin{array}{l}220 \\
3\end{array}$ & $\begin{array}{l}44 . \\
7\end{array}$ & $\begin{array}{l}251 . \\
6\end{array}$ & $\begin{array}{l}5 . \\
5\end{array}$ & $\begin{array}{l}254 . \\
9\end{array}$ & \\
\hline K264@15 & $\begin{array}{l}10 \\
39\end{array}$ & 148 & 46 & $\begin{array}{l}0.1 \\
42\end{array}$ & $\begin{array}{l}1773 \\
4\end{array}$ & $\begin{array}{l}\{0.1 \\
1\}\end{array}$ & $\begin{array}{l}0.28 \\
819\end{array}$ & $\begin{array}{l}2 . \\
32\end{array}$ & $\begin{array}{l}0.04 \\
04\end{array}$ & $\begin{array}{l}1 . \\
50\end{array}$ & $\begin{array}{l}0.05 \\
169\end{array}$ & $\begin{array}{l}1.2 \\
77\end{array}$ & $\begin{array}{l}271 . \\
6\end{array}$ & $\begin{array}{l}40 \\
1\end{array}$ & $\begin{array}{l}257 . \\
1\end{array}$ & $\begin{array}{l}5 . \\
3\end{array}$ & & $\begin{array}{l}3 . \\
8\end{array}$ \\
\hline 'K264@16 & $\begin{array}{l}12 \\
47\end{array}$ & 379 & 57 & $\begin{array}{l}0.3 \\
04\end{array}$ & $\begin{array}{l}6725 \\
3\end{array}$ & $\begin{array}{l}\{0.0 \\
3\}\end{array}$ & $\begin{array}{l}0.29 \\
063\end{array}$ & $\begin{array}{l}1 . \\
84\end{array}$ & $\begin{array}{l}0.04 \\
00\end{array}$ & $\begin{array}{l}1 . \\
50\end{array}$ & $\begin{array}{l}0.05 \\
266\end{array}$ & $\begin{array}{l}1.3 \\
06\end{array}$ & $\begin{array}{l}314 . \\
0\end{array}$ & $\begin{array}{l}23 \\
9\end{array}$ & & $\begin{array}{l}4 . \\
2\end{array}$ & & $\begin{array}{l}3 . \\
7\end{array}$ \\
\hline [K264@17 & $\begin{array}{l}45 \\
4\end{array}$ & 172 & 21 & $\begin{array}{l}0.3 \\
79\end{array}$ & 9931 & $\begin{array}{l}\{0.1 \\
9\}\end{array}$ & $\begin{array}{l}0.27 \\
462\end{array}$ & $\begin{array}{l}2 . \\
51\end{array}$ & $\begin{array}{l}0.03 \\
96\end{array}$ & $\begin{array}{l}1 . \\
50\end{array}$ & $\begin{array}{l}0.05 \\
028\end{array}$ & $\begin{array}{l}2 . \\
01\end{array}$ & $\begin{array}{l}207 . \\
8\end{array}$ & $\begin{array}{l}46 \\
1\end{array}$ & $\begin{array}{l}246 . \\
4\end{array}$ & $\begin{array}{l}5 . \\
5\end{array}$ & $\begin{array}{l}250 . \\
4\end{array}$ & $\begin{array}{l}3 . \\
7\end{array}$ \\
\hline TK264@18* & $\begin{array}{l}17 \\
79\end{array}$ & 742 & 86 & $\begin{array}{l}0.4 \\
17\end{array}$ & $\begin{array}{l}4734 \\
3\end{array}$ & $\begin{array}{l}\{0.0 \\
4\}\end{array}$ & $\begin{array}{l}0.28 \\
913\end{array}$ & $\begin{array}{l}1 . \\
78\end{array}$ & $\begin{array}{l}0.04 \\
12\end{array}$ & & $\begin{array}{l}0.05 \\
089\end{array}$ & $\begin{array}{l}0.2 \\
96\end{array}$ & $\begin{array}{l}235 . \\
9\end{array}$ & $\begin{array}{l}22 . \\
0\end{array}$ & $\begin{array}{l}257 . \\
9\end{array}$ & $\begin{array}{l}4 \\
1\end{array}$ & $\begin{array}{l}260 . \\
3\end{array}$ & $\begin{array}{l}3 \\
8\end{array}$ \\
\hline ГK264@19 & $\begin{array}{l}60 \\
1\end{array}$ & 276 & 28 & $\begin{array}{l}0.4 \\
59\end{array}$ & $\begin{array}{l}1007 \\
37\end{array}$ & $\begin{array}{l}\{0.0 \\
2\}\end{array}$ & & $\begin{array}{l}2 . \\
15\end{array}$ & $\begin{array}{l}0.03 \\
93\end{array}$ & & $\begin{array}{l}0.05 \\
069\end{array}$ & $\begin{array}{l}1.2 \\
53\end{array}$ & $\begin{array}{l}226 . \\
7\end{array}$ & $\begin{array}{l}34 . \\
9\end{array}$ & $\begin{array}{l}246 . \\
6\end{array}$ & $\begin{array}{l}4 . \\
7\end{array}$ & $\begin{array}{l}248 . \\
7\end{array}$ & $\begin{array}{l}3 . \\
7\end{array}$ \\
\hline ГK264@20 & $\begin{array}{l}55 \\
1\end{array}$ & 301 & 26 & $\begin{array}{l}0.5 \\
46\end{array}$ & $\begin{array}{l}1235 \\
1\end{array}$ & $\begin{array}{l}\{0.1 \\
5\}\end{array}$ & $\begin{array}{l}0.27 \\
453\end{array}$ & $\begin{array}{l}2 . \\
75\end{array}$ & $\begin{array}{l}0.03 \\
93\end{array}$ & & $\begin{array}{l}0.05 \\
066\end{array}$ & $\begin{array}{l}2 . \\
31\end{array}$ & $\begin{array}{l}225 . \\
4\end{array}$ & $\begin{array}{l}52 \\
4\end{array}$ & $\begin{array}{l}246 . \\
3\end{array}$ & $\begin{array}{l}6 . \\
0\end{array}$ & $\begin{array}{l}248 . \\
5\end{array}$ & $\begin{array}{l}3 . \\
7\end{array}$ \\
\hline TK264@21 & $\begin{array}{l}56 \\
5\end{array}$ & 205 & 26 & $\begin{array}{l}0.3 \\
63\end{array}$ & & $\begin{array}{l}\{0.0 \\
0\}\end{array}$ & $\begin{array}{l}0.28 \\
198\end{array}$ & $\begin{array}{l}2 . \\
15\end{array}$ & $\begin{array}{l}0.03 \\
94\end{array}$ & & $\begin{array}{l}0.05 \\
193\end{array}$ & $\begin{array}{l}1.2 \\
542\end{array}$ & & $\begin{array}{l}34 \\
8\end{array}$ & $\begin{array}{l}252 . \\
2\end{array}$ & $\begin{array}{l}4 . \\
8\end{array}$ & $\begin{array}{l}249 . \\
0\end{array}$ & 3 \\
\hline K264@2 & & 590 & 65 & $\begin{array}{l}0.4 \\
34\end{array}$ & $\begin{array}{l}4427 \\
1\end{array}$ & $\begin{array}{l}\{0.0 \\
4\}\end{array}$ & $\begin{array}{l}0.28 \\
678\end{array}$ & $\begin{array}{l}1 . \\
97\end{array}$ & $\begin{array}{l}0.0 \\
05\end{array}$ & & $\begin{array}{l}0.05 \\
138\end{array}$ & $26^{2}$ & & & & & & \\
\hline
\end{tabular}

\subsection{SIMS zircon oxygen isotope measurement}

Samples for zircon oxygen isotopes were measured using the Cameca IMS-1280 SIMS at the Institute of Geology and Geophysics in the Chinese Academy of Sciences, Beijing. The original mounts were re-ground and polished to remove any trace of the analytical pits after $\mathrm{U}-\mathrm{Pb}$ dating. The $\mathrm{Cs}+$ primary ion beam was accelerated at $10 \mathrm{kV}$, with an intensity of ca.2 $\mathrm{nA}$ (Gaussian mode with a primary beam aperture of $200 \mu \mathrm{m}$ to reduce aberrations) and rastered over a $10 \mu \mathrm{m}$ area. The spot size is about $20 \mu \mathrm{m}$ in diameter. The normal incidence electron flood gun was used to compensate for sample charging during analysis with homogeneous electron density over a $100 \mu \mathrm{m}$ oval area. $60 \mathrm{ev}$ energy window was used, together with a mass resolution of ca.2500. Oxygen isotopes were measured using multicollection mode on two off-axis Faraday cups. The intensity of ${ }^{16} \mathrm{O}$ was typically $1 \times 109 \mathrm{cps}$. The NMR (Nuclear Magnetic Resonance) probe was used for magnetic field control with stability better than $3 \mathrm{ppm}$ over $16 \mathrm{~h}$ on mass 17 . One analysis takes ca. 5 min consisting of pre-sputtering $(\sim 120 \mathrm{~s})$, automatic beam centering $(\sim 60 \mathrm{~s})$ and integration of oxygen isotopes (20 cycles $\times 4 \mathrm{~s}$, total $80 \mathrm{~s}$ ). Uncertainties on individual analyses are usually better than $0.2-$ $0.3 \%$ o $(1 \sigma)$.

The instrumental mass fractionation (IMF) factor is corrected using zircon 91,500 standard with a $\delta^{18} \mathrm{O}$ value of $9.9 \pm 0.3 \%$ (Wiedenbeck et al., 2004) and Penglai zircon standard $\left(\delta^{18} \mathrm{O}\right.$ VSMOW $=5.3 \%$ ) ( $\mathrm{Li}$ et al., 2010). The internal precision of a single analysis generally was better than $0.2 \%$ o $(1 \sigma$ standard error $)$ for the ${ }^{18} \mathrm{O} /{ }^{16} \mathrm{O}$ ratio. Measured ${ }^{18} \mathrm{O} /{ }^{16} \mathrm{O}$ ratios were normalized by using Vienna Standard Mean Ocean Water compositions (VSMOW, $\left.{ }^{18} \mathrm{O} /{ }^{16} \mathrm{O}=0.0020052\right)$, and then corrected for the instrumental mass fractionation factor (IMF) as follows: 
$\left(\delta^{18} \mathrm{O}\right) \mathrm{M}\left[\frac{\left({ }^{18} \mathrm{O} /{ }^{16} \mathrm{O}\right) \mathrm{M}}{0.0020052}-1\right] \times 1000(\%)$.

IMF $=\left(\delta^{18} \mathrm{O}\right)_{\text {M(standard) }}-\left(\delta^{18} \mathrm{O}\right)_{\text {vsmow, }}$

$\delta^{18} O_{\text {sample }}=\left(\delta^{18} O\right)_{M}+1 M F$

Zircon oxygen isotopic data are listed in Table 3.

Table 3. : In situ zircon Hf-O isotopic results.

\section{Spot $^{\mathrm{a}} \quad{ }^{176} \mathrm{Lu} /{ }^{177} \mathbf{H f}{ }^{176} \mathrm{Hf} /{ }^{177} \mathrm{Hf} \quad \pm 2 \sigma_{m} \quad \varepsilon_{\mathrm{Hf}}(t) \mathrm{T}_{\mathrm{DM}}{ }^{\mathrm{C}}(\mathrm{Ma}) \delta^{18} \mathrm{O}(\%) \pm 2 \sigma_{m}$} $\mathrm{Co}^{`}$ Linh pluton (Granodiorite TK61, 254.8 Ma)

$\begin{array}{ccccccc}\text { TK61@ } 1 & 0.001737 & 0.282497 & 0.000018-4.3 & 1563 & 9.20 & 0.32 \\ \text { TK61@2 } & 0.001106 & 0.282498 & 0.000017-4.3 & 1558 & 9.26 & 0.38 \\ \text { TK61@3 } & 0.000866 & 0.282500 & 0.000017-4.2 & 1552 & 9.61 & 0.24 \\ \text { TK61@4 } & 0.001853 & 0.282515 & 0.000018-3.8 & 1527 & 9.54 & 0.20 \\ \text { TK61@5 } & 0.001476 & 0.282486 & 0.000015-4.6 & 1583 & 8.46 & 0.18 \\ \text { TK61@6 } & 0.001427 & 0.282532 & 0.000017-3.0 & 1482 & 9.62 & 0.27 \\ \text { TK61@7 } & 0.003722 & 0.282527 & 0.000021-3.8 & 1521 & 8.79 & 0.36 \\ \text { TK61@8 } & 0.001917 & 0.282513 & 0.000019-3.9 & 1532 & 9.95 & 0.37 \\ \text { TK61@9 } & 0.001289 & 0.282544 & 0.000014-2.7 & 1456 & 9.29 & 0.32 \\ \text { TK61@10 } & 0.001794 & 0.282501 & 0.000019-4.7 & 1567 & 9.86 & 0.24 \\ \text { TK61@12 } & 0.001335 & 0.282514 & 0.000017-3.6 & 1520 & 9.43 & 0.34 \\ \text { TK61@14 } & 0.001739 & 0.282513 & 0.000018-3.7 & 1527 & 9.47 & 0.26 \\ \text { TK61@16 } & 0.001805 & 0.282475 & 0.000019-5.1 & 1614 & 9.51 & 0.31 \\ \text { TK61@17 } & 0.002172 & 0.282566 & 0.000019-2.1 & 1417 & 9.15 & 0.37 \\ \text { TK61@18 } & 0.001561 & 0.282473 & 0.000020-5.2 & 1618 & 9.47 & 0.39 \\ \text { TK61@19 } & 0.000972 & 0.282511 & 0.000018-3.7 & 1525 & 9.12 & 0.25 \\ \text { TK61@20 } & 0.001520 & 0.282526 & 0.000020-3.3 & 1497 & 9.65 & 0.28 \\ \text { TK61@21 } & 0.001473 & 0.282512 & 0.000020-3.9 & 1531 & 9.59 & 0.20 \\ \text { TK61@22 } & 0.001417 & 0.282531 & 0.000020-3.0 & 1483 & 8.86 & 0.28 \\ \text { TK61@23 } & 0.001449 & 0.282512 & 0.000017-3.7 & 1524 & 9.05 & 0.27\end{array}$

Nà Giao pluton (Granites TK84,TK85, $90 \mathrm{Ma}$ )

$\begin{array}{lllllll}\text { TK84@2 } & 0.001459 & 0.282534 & 0.000016-6.5 & 1570 & 8.19 & 0.34 \\ \text { TK84@3 } & 0.001112 & 0.282467 & 0.000011-8.9 & 1719 & 9.87 & 0.16 \\ \text { TK84@6 } & 0.002208 & 0.282643 & 0.000015-2.7 & 1330 & 8.69 & 0.30 \\ \text { TK84@8 } & 0.001121 & 0.282529 & 0.000013-6.7 & 1580 & 9.52 & 0.30 \\ \text { TK84@9 } & 0.001013 & 0.282580 & 0.000014-4.9 & 1466 & 8.17 & 0.25 \\ \text { TK84@12 } & 0.000717 & 0.282424 & 0.000016-10.4 & 1812 & 9.17 & 0.22\end{array}$




\begin{tabular}{|c|c|c|c|c|c|c|}
\hline Spot $^{-\mathbf{a}}$ & $\mathbf{L u} / \mathbf{n}$ & ${ }^{6} \mathbf{H f} /{ }^{177} \mathbf{H}$ & $\pm 2 \sigma_{m} \quad \varepsilon_{\mathrm{Hf}}(t)$ & $\mathbf{T}_{\mathrm{DM}}^{\mathrm{C}}(\mathbf{M}$ & $\delta^{18} \mathbf{O}$ & $\pm 2 \sigma_{1}$ \\
\hline ГK84@ 14 & 0.001407 & 0.282456 & $0.000013-9.3$ & 1744 & 9.38 & 0.23 \\
\hline TK85@4 & 0.001326 & 0.282494 & $0.000016-7.8$ & 1654 & 9.95 & 0.30 \\
\hline TK85@6 & 0.000778 & 0.282508 & $0.000012-7.4$ & 1625 & 10.95 & 0.37 \\
\hline TK85@9 & 0.002766 & 0.282564 & $0.000022-5.5$ & 1508 & 9.54 & 0.22 \\
\hline TK85@10 & 0.001259 & 0.282464 & $0.000015-9.0$ & 1724 & 9.78 & 0.21 \\
\hline TK85@11 & 0.000841 & 0.282383 & $0.000015-11.8$ & 1903 & 8.12 & 0.24 \\
\hline TK85@12 & 0.000257 & 0.282478 & $0.000016-8.5$ & 1692 & 9.34 & 0.23 \\
\hline TK85@13 & 0.000667 & 0.282552 & $0.000017-5.8$ & 1526 & 9.39 & 0.26 \\
\hline TK85@17 & 0.000150 & 0.282481 & $0.000016-8.4$ & 1687 & 9.55 & 0.25 \\
\hline TK85@18 & 0.000336 & 0.282463 & $0.000015-9.0$ & 1727 & 9.89 & 0.35 \\
\hline TK85@20 & 0.000438 & 0.282536 & $0.000017-6.4$ & 1565 & 8.24 & 0.29 \\
\hline TK85@21 & 0.000115 & 0.282529 & $0.000014-6.7$ & 1579 & 8.28 & 0.23 \\
\hline TK85@24 & 0.000695 & 0.282532 & $0.000013-6.6$ & 1574 & 8.54 & 0.26 \\
\hline @ 25 & 0.000274 & 0.282449 & $0.000016-9.5$ & 1758 & 9.92 & 0.34 \\
\hline @ 26 & 0.000058 & 0.282530 & $0.000023-6.6$ & 1578 & 9.71 & 0.23 \\
\hline TK85@28 & 0.001598 & 0.282521 & $0.000015-7.0$ & 1600 & 8.42 & 0.44 \\
\hline TK85@29 & 0.000006 & 0.282463 & $0.000013-9.0$ & 1724 & 9.62 & 0.31 \\
\hline \multicolumn{7}{|c|}{ Hong Thái pluton (Quartz-syenite TK164, $245 \mathrm{Ma}$ ) } \\
\hline TK164@1 & 0.000718 & 0.282619 & $0.000027-0.2$ & 1289 & 4.61 & 0.29 \\
\hline TK164@2 & 0.000646 & 0.282574 & $0.000028-1.7$ & 1387 & 4.14 & 0.31 \\
\hline TK164@4 & 0.000894 & 0.282558 & $0.000023-2.3$ & 1426 & 4.04 & 0.30 \\
\hline TK164@5 & 0.001974 & 0.282567 & $0.000027-2.2$ & 1417 & 3.99 & 0.30 \\
\hline TK164@8 & 0.001609 & 0.282566 & $0.000025-2.2$ & 1417 & 4.74 & 0.35 \\
\hline TK164@10 & 0.000588 & 0.282563 & $0.000022-2.1$ & 1411 & 4.09 & 0.30 \\
\hline TK164@12 & 0.000867 & 0.282460 & $0.000026-5.5$ & 1637 & 4.32 & 0.35 \\
\hline TK164@13 & 0.001159 & 0.282576 & $0.000032-1.7$ & 1389 & 4.06 & 0.39 \\
\hline TK164@14 & 0.001920 & 0.282529 & $0.000027-3.6$ & 1503 & 4.14 & 0.21 \\
\hline TK164@15 & 0.001020 & 0.282554 & $0.000026-2.5$ & 1437 & 3.99 & 0.24 \\
\hline TK164@16 & 0.000393 & 0.282428 & $0.000026-6.8$ & 1712 & 4.33 & 0.45 \\
\hline TK164@18 & 0.000771 & 0.282541 & $0.000023-3.0$ & 1465 & 4.39 & 0.33 \\
\hline \multicolumn{7}{|c|}{ Hong Thái pluton (Quartz-syenite TK164, 525 Ma) } \\
\hline 4@3 & 0.000922 & 0.282497 & 0.0000191 .5 & 1399 & 4.63 & 0.35 \\
\hline TK164@6 & 0.001249 & 0.282524 & 0.0000292 .3 & 1347 & 5.26 & 0.16 \\
\hline TK164@7 & 0.001208 & 0.282512 & 0.0000311 .8 & 1377 & 4.62 & 0.19 \\
\hline TK164@9 & 0.000631 & 0.282476 & 0.0000260 .8 & 1443 & 4.32 & 0.26 \\
\hline TK164@11 & 0.000725 & 0.282452 & 0.0000280 .2 & 1490 & 4.64 & 0.33 \\
\hline TK164@17 & 0.001029 & 0.282546 & 0.0000293 .1 & 1294 & 5.13 & 0.29 \\
\hline TK164@1 & 0.001223 & 0.282492 & 0.0000281 .2 & 1418 & 4.38 & 0.30 \\
\hline TK164@2 & 0.000648 & 0.282527 & 0.0000272 .8 & 1323 & 4.56 & 0.32 \\
\hline
\end{tabular}

Bach Ha pluton (Biotite granite TK216, 227.7 Ma) 


\begin{tabular}{|c|c|c|c|c|c|c|}
\hline Spot ${ }^{\mathbf{a}}$ & $\mathbf{u} /{ }^{177}$ & ${ }^{6} \mathbf{H f} /{ }^{177} \mathbf{H}$ & $\varepsilon_{\mathrm{Hf}}(t)$ & $\mathbf{T}_{\mathrm{DM}}^{\mathrm{C}}(\mathbf{M}$ & $\delta^{18} \mathbf{O}$ & $\pm 2 \sigma_{m}$ \\
\hline K216@1 & 0.001427 & 0.282362 & $0.000016-9.4$ & 1869 & 9.62 & 0.23 \\
\hline TK216@2 & 0.001957 & 0.282423 & $0.000016-7.1$ & 1734 & 9.79 & 0.30 \\
\hline TK216@4 & 0.001724 & 0.282397 & $0.000016-8.2$ & 1795 & 10.23 & 0.28 \\
\hline TK216@8 & 0.001841 & 0.282425 & $0.000019-7.0$ & 1728 & 9.42 & 0.31 \\
\hline TK216@9 & 0.001805 & 0.282453 & $0.000019-6.0$ & 1666 & 9.68 & 0.42 \\
\hline TK216@10 & 0.002722 & 0.282423 & $0.000020-7.3$ & 1745 & 9.46 & 0.27 \\
\hline TK216@12 & 0.002359 & 0.282423 & $0.000018-7.1$ & 1737 & 9.70 & 0.27 \\
\hline TK216@13 & 0.002487 & 0.282448 & $0.000020-6.4$ & 1688 & 9.37 & 0.28 \\
\hline TK216@16 & 0.002749 & 0.282409 & $0.000022-7.7$ & 1774 & 8.94 & 0.28 \\
\hline TK216@17 & 0.001735 & 0.282394 & $0.000021-8.1$ & 1798 & 9.08 & 0.41 \\
\hline TK216@18 & 0.002514 & 0.282420 & $0.000020-7.2$ & 1745 & 9.65 & 0.32 \\
\hline TK216@19 & 0.002794 & 0.282426 & $0.000016-7.1$ & 1736 & 9.32 & 0.39 \\
\hline TK216@20 & 0.00 & 0.282399 & 0.000 & 1784 & 5 & 0.31 \\
\hline \multicolumn{7}{|c|}{ Drem Mac pluton (Granodiorite TK264, 251.8 Ma) } \\
\hline TK264@2 & 0.000953 & 0.282375 & $0.000013-8.6$ & 1829 & 11.08 & 0.25 \\
\hline TK264@3 & 0.000701 & 0.282354 & $0.000014-9.5$ & 1879 & 10.74 & 0.23 \\
\hline TK264@4 & 0.000875 & 0.282337 & $0.000014-10.0$ & 1915 & 11.15 & 0.34 \\
\hline TK264@5 & 0.000978 & 0.282389 & $0.000013-8.2$ & 1800 & 11.59 & 0.30 \\
\hline TK264@6 & 0.000704 & 0.282381 & $0.000012-8.5$ & 1816 & 11.41 & 0.19 \\
\hline TK264@7 & 0.001064 & 0.282421 & $0.000013-7.0$ & 1728 & 12.69 & 0.27 \\
\hline TK264@9 & 0.001247 & 0.282408 & $0.000014-7.7$ & 1766 & 11.47 & 0.40 \\
\hline TK264@10 & 0.000628 & 0.282390 & $0.000012-8.1$ & 1795 & 12.07 & 0.32 \\
\hline TK264@1 & 0.001243 & 0.282413 & $0.000012-7.2$ & 1746 & 10.83 & 0.24 \\
\hline TK264@12 & 0.000824 & 0.282379 & $0.000013-8.5$ & 1822 & 11.25 & 0.21 \\
\hline TK264@1. & 0.001300 & 0.282380 & $0.000014-8.5$ & 1822 & 12.17 & 0.25 \\
\hline TK264@1. & 0.001080 & 0.282393 & $0.000013-8.0$ & 1791 & 11.31 & 0.30 \\
\hline TK264@1 & 000914 & 0.282415 & $0.000015-7.2$ & 1741 & 10.61 & 0.28 \\
\hline TK264@16 & 0.001266 & 0.282411 & $0.000012-7.4$ & 1755 & 10.88 & 0.29 \\
\hline TK264@1 & 0.000919 & 0.282371 & $0.000012-8.8$ & 1841 & 10.74 & 0.20 \\
\hline TK264@1 & 0.001517 & 0.282357 & $0.000014-9.2$ & 1873 & 10.71 & 0.20 \\
\hline TK264@19 & 0.001079 & 0.282395 & $0.000010-8.1$ & 1790 & 11.53 & 0.26 \\
\hline TK264@20 & 0.001021 & 0.282342 & $0.000012-9.9$ & 1908 & 11.13 & 0.31 \\
\hline TK264@21 & 0.001026 & 0.282402 & $0.000013-7.8$ & 1773 & 11.49 & 0.32 \\
\hline TK264@22 & 0.000930 & 0.282458 & $0.000013-5.6$ & 1644 & 12.81 & 0.35 \\
\hline
\end{tabular}

The spots with asterisk do not calculate as concordia age.

a

The analysis were conducted in the same spot as the SIMS U-Pb spot. $\varepsilon_{\mathrm{Hf}}(\mathrm{T})=\left[{ }^{176} \mathrm{Hf} /{ }^{177} \mathrm{Hf}_{\mathrm{Z}} /{ }^{176} \mathrm{Hf} /{ }^{177} \mathrm{Hf}_{\mathrm{CHUR}(\mathrm{T})}-1\right] \times 10,000$; 
${ }^{176} \mathrm{Hf} /{ }^{177} \mathrm{Hf}$ CHUR(T) $={ }^{176} \mathrm{Hf} /{ }^{177} \mathrm{Hf}_{\mathrm{CHUR}(0)}{ }^{176} \mathrm{Lu} /{ }^{177} \mathrm{Hf}_{\mathrm{CHUR}} \times\left(\mathrm{e}^{\lambda \mathrm{T}}-1\right)$; $\mathrm{T}_{\mathrm{DM}}=(1 / \lambda) \times \ln \left[1+\left({ }^{176} \mathrm{Hf} /{ }^{177} \mathrm{Hf}_{\mathrm{DM}}{ }^{-176} \mathrm{Hf}^{177} \mathrm{Hf}_{\mathrm{Z}}\right) /\left({ }^{176} \mathrm{Lu} /{ }^{177} \mathrm{Hf}_{\mathrm{DM}}-{ }^{176} \mathrm{Lu} /{ }^{177} \mathrm{Hf}_{\mathrm{Z}}\right)\right]$; $\mathrm{T}_{\mathrm{DM}}{ }^{\mathrm{C}}=\mathrm{T}_{\mathrm{DM}}-\left(\mathrm{T}_{\mathrm{DM}}-\mathrm{T}\right) \times\left[\left(\mathrm{f}_{\mathrm{CC}}-\mathrm{f}_{\mathrm{Z}}\right) /\left(\mathrm{f}_{\mathrm{CC}}-\mathrm{f}_{\mathrm{DM}}\right)\right] ; \mathrm{f}_{\mathrm{Lu} / \mathrm{Hf}}={ }^{176} \mathrm{Hf} /{ }^{177} \mathrm{Hf}_{\mathrm{Z}} /{ }^{176} \mathrm{Lu} /{ }^{177} \mathrm{Hf}_{\mathrm{CHUR}}-1$, where $f_{C C}, f_{Z}$ and $f_{D M}$ are the $f_{L u / H f}$ values of the continental crust, zircon sample and the depleted mantle; subscript $\mathrm{Z}=$ analyzed zircon sample, $\mathrm{CHUR}=$ chondritic uniform reservoir; $\mathrm{DM}=$ depleted mantle; $\mathrm{T}$ is timing of the granitoids crystallization; $\lambda=1.867 \times 10-11$ year-1, decay constant of ${ }^{176} \mathrm{Lu}$ (Söderlund et al., 2004); ${ }^{176} \mathrm{Hf} /{ }^{177} \mathrm{Hf} \mathrm{f}_{\mathrm{DM}}=0.28325 ;{ }^{176} \mathrm{Lu} /{ }^{177} \mathrm{Hf}_{\mathrm{DM}}=0.0384$; present-day ${ }^{176} \mathrm{Hf} /{ }^{177} \mathrm{Hf} \mathrm{CHUR}_{\mathrm{C}(0)}=$ $0.282772 ;{ }^{176} \mathrm{Hf} /{ }^{177} \mathrm{Hf}_{\mathrm{CHUR}}=0.0332 ;{ }^{176} \mathrm{Hf} /{ }^{177} \mathrm{Hf}_{\mathrm{CC}}=0.015 . \mathrm{T}_{\mathrm{DM}}$ represents the model age calculated from the measured ${ }^{176} \mathrm{Hf} /{ }^{177} \mathrm{Hf}$ and ${ }^{176} \mathrm{Lu} /{ }^{177} \mathrm{Hf}$ ratios of a zircon, giving a minimum limit for the crustal residence age of the hafnium in the zircon; whereas $\mathrm{T}_{\mathrm{DM}}{ }^{\mathrm{C}}$, a "crust Hf model age," is derived from projecting the initial ${ }^{176} \mathrm{Hf} /{ }^{177} \mathrm{Hf}$ of a zircon with a $\mathrm{Lu} / \mathrm{Hf}$ ratio corresponding to the continental crust back to the depleted mantle model growth curve. Thus, $\mathrm{T}_{\mathrm{DM}}{ }^{\mathrm{C}}$ can represent the mean crustal residence age of the source material extracted from the depleted mantle.

\subsection{LA-MC-ICPMS zircon Lu-Hf isotope measurements}

In situ zircon Lu-Hf isotopic analysis was carried out on a Neptune multi-collector ICPMS equipped with a Geolas-193 laser-ablation system (LA-MC-ICPMS) at the Institute of Geology and Geophysics, Beijing. Lu-Hf isotopic measurements were made on the same zircon grains previously analyzed for $\mathrm{U}-\mathrm{Pb}$ and $\mathrm{O}$ isotopes, with ablation pit of $63 \mu \mathrm{m}$ in diameter, repetition rate of $8-10 \mathrm{~Hz}$, laser beam energy density of $10 \mathrm{~J} / \mathrm{cm}^{2}$, and ablation time of $26 \mathrm{~s}$. The detailed analytical procedures were similar to those described by $\mathrm{Wu}$ et al. (2006). Contribution of isobaric interferences by ${ }^{176} \mathrm{Lu}$ and ${ }^{176} \mathrm{Yb}$ on the ${ }^{176} \mathrm{Hf}$ signal were subtracted by monitoring the intensity of ${ }^{175} \mathrm{Lu}$ and ${ }^{172} \mathrm{Yb}$ signals, using ${ }^{176} \mathrm{Lu} /{ }^{175} \mathrm{Lu}=0.026549$ and ${ }^{176} \mathrm{Yb} /{ }^{172} \mathrm{Yb}=0.5886$ (Chu et al., 2002). Independent mass bias factors for $\mathrm{Hf}$ and $\mathrm{Yb}\left(\beta_{\mathrm{Hf}}\right.$ and $\left.\beta_{\mathrm{Yb}}\right)$ in the isobaric interference correction were used. Measured ${ }^{176} \mathrm{Hf} /{ }^{177} \mathrm{Hf}$ ratios were normalized to ${ }^{179} \mathrm{Hf} /{ }^{177} \mathrm{Hf}=0.7325$. Further external adjustment is not applied for the unknowns because our determined ${ }^{176} \mathrm{Hf} /{ }^{177} \mathrm{Hf}$ ratios of $0.282303 \pm 0.000020$ for zircon standards 91,500 are in good agreement with the reported values (Wu et al., 2006a). All the Lu-Hf isotope analysis results are listed in Table 3 with the error in $2 \sigma$ of the mean.

\subsection{Bulk-rock Sr-Nd isotopic analysis}

$\mathrm{Sr}$ and Nd isotopic compositions were measured on a Finnigan Mat 262 thermal ionization mass spectrometer at the Institute of Geology and Geophysics in the Chinese Academy of Sciences, Beijing, following the procedure described in Zhang et al. (2008). Procedural blanks were $<100 \mathrm{pg}$ for Sm and $\mathrm{Nd}$ and $<500 \mathrm{pg}$ for $\mathrm{Rb}$ and Sr. ${ }^{143} \mathrm{Nd} /{ }^{144} \mathrm{Nd}$ was corrected for mass fractionation by normalization to ${ }^{146} \mathrm{Nd} /{ }^{144} \mathrm{Nd}=0.7219$, and ${ }^{87} \mathrm{Sr} /{ }^{86} \mathrm{Sr}$ ratios were normalized to ${ }^{86} \mathrm{Sr} /{ }^{88} \mathrm{Sr}=0.1194$. The measured values for the NBS-987 $\mathrm{Sr}$ standard were ${ }^{87} \mathrm{Sr} /{ }^{86} \mathrm{Sr}=0.710228 \pm 0.000010\left(2 \sigma_{\mathrm{m}}\right)$ during the period of data acquisition. Bulk-rock $\mathrm{Sr}-\mathrm{Nd}$ isotopic data are listed in Table 4

Table 4. : Bulk-rock Sr-Nd isotopic results.

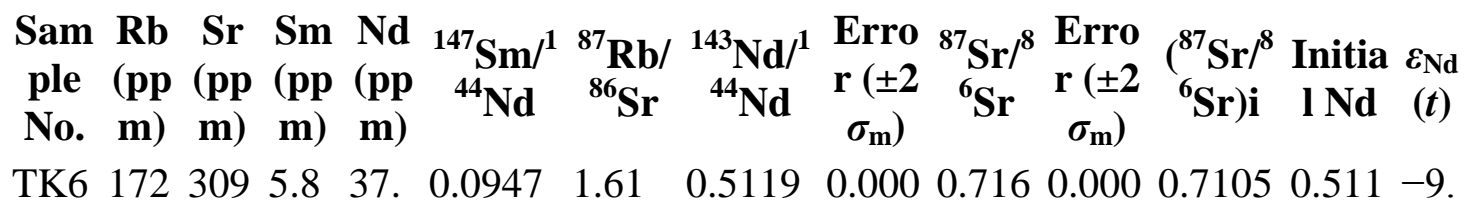




\begin{tabular}{|c|c|c|c|c|c|c|c|c|c|c|c|c|c|}
\hline $\begin{array}{c}\text { Sam } \\
\text { ple } \\
\text { No. }\end{array}$ & $\begin{array}{l}\mathbf{R b} \\
\text { (pp } \\
\mathbf{m})\end{array}$ & $\begin{array}{l}\text { Sr } \\
\text { (pp } \\
\text { m) }\end{array}$ & $\begin{array}{l}\text { Sm } \\
\text { (pp } \\
\text { m) }\end{array}$ & $\begin{array}{l}\text { Nd } \\
\text { (pp } \\
\text { m) }\end{array}$ & $\begin{array}{c}{ }^{147} \mathrm{Sm} /{ }^{1} \\
{ }^{44} \mathrm{Nd}\end{array}$ & ${ }^{{ }^{86}} \mathbf{R b} \mathrm{Sr} /$ & $\begin{array}{c}{ }^{143} \mathrm{Nd} /{ }^{1} \\
{ }^{44} \mathrm{Nd}\end{array}$ & $\begin{array}{c}\text { Erro } \\
\mathbf{r}( \pm 2 \\
\left.\sigma_{\mathrm{m}}\right)\end{array}$ & $\begin{array}{c}{ }^{87} \mathrm{Sr} /{ }^{8} \\
{ }^{6} \mathrm{Sr}\end{array}$ & $\begin{array}{c}\text { Erro } \\
\mathbf{r}( \pm 2 \\
\left.\sigma_{\mathrm{m}}\right)\end{array}$ & $\begin{array}{c}\left({ }^{87} \mathrm{Sr} / /^{8}\right. \\
\left.{ }^{6} \mathrm{Sr}\right) \mathrm{i}\end{array}$ & $\begin{array}{l}\text { Initia } \\
\text { I Nd }\end{array}$ & $\begin{array}{c}\varepsilon_{\mathrm{Nd}} \\
(t)\end{array}$ \\
\hline 1 & .3 & .9 & 8 & 6 & & & 59 & 014 & 363 & 011 & & 802 & 7 \\
\hline $\begin{array}{l}\text { TK8 } \\
4\end{array}$ & $\begin{array}{l}665 \\
.9\end{array}$ & $\begin{array}{l}39 . \\
0\end{array}$ & $\begin{array}{l}3.3 \\
5\end{array}$ & $\begin{array}{l}15 . \\
2\end{array}$ & 0.1333 & 49.5 & $\begin{array}{l}0.5120 \\
34\end{array}$ & $\begin{array}{l}0.000 \\
015\end{array}$ & $\begin{array}{l}0.788 \\
359\end{array}$ & $\begin{array}{l}0.000 \\
013\end{array}$ & 0.7251 & $\begin{array}{l}0.511 \\
956\end{array}$ & $\begin{array}{l}-1 \\
1.0\end{array}$ \\
\hline $\begin{array}{l}\text { TK8 } \\
5\end{array}$ & $\begin{array}{l}648 \\
.5\end{array}$ & $\begin{array}{l}39 . \\
9\end{array}$ & $\begin{array}{l}3.6 \\
7\end{array}$ & $\begin{array}{l}16 . \\
2\end{array}$ & 0.1370 & 47.0 & $\begin{array}{l}0.5120 \\
61\end{array}$ & $\begin{array}{l}0.000 \\
015\end{array}$ & $\begin{array}{l}0.788 \\
476\end{array}$ & $\begin{array}{l}0.000 \\
011\end{array}$ & 0.728 & & \\
\hline
\end{tabular}

Notes: $\mathrm{I}_{\mathrm{Sr}}=\left({ }^{87} \mathrm{Sr} /{ }^{86} \mathrm{Sr}\right)_{\text {sample }}-\left({ }^{87} \mathrm{Rb} /{ }^{86} \mathrm{Sr}\right)_{\text {sample }} \times\left(\mathrm{e}^{\lambda \mathrm{t}}-1\right), \lambda=1.42 \times 10-11$ year -1 (Steiger and Jäger, 1977); initial $\mathrm{Nd}=\left({ }^{143} \mathrm{Nd} /{ }^{144} \mathrm{Nd}\right)_{\text {sample }}-\left({ }^{147} \mathrm{Sm} /{ }^{144} \mathrm{Nd}\right)_{\text {sample }} \times\left(\mathrm{e}^{\lambda \mathrm{t}}-1\right)$, $\varepsilon_{\mathrm{Nd}}=\left(\left({ }^{143} \mathrm{Nd} /{ }^{144} \mathrm{Nd}\right)_{\text {sample }} /\left({ }^{143} \mathrm{Nd} /{ }^{144} \mathrm{Nd}\right)_{\mathrm{CHUR}}-1\right) \times 10000$, $\mathrm{f}_{\mathrm{Sm} / \mathrm{Nd}}=\left({ }^{147} \mathrm{Sm} /{ }^{144} \mathrm{Sm}\right)_{\mathrm{sample}} /\left({ }^{147} \mathrm{Sm} /{ }^{144} \mathrm{Sm}\right)_{\mathrm{CHUR}}-1$, $\mathrm{T}_{\mathrm{DM} 1}=1 / \lambda \times \ln \left(1+\left(\left({ }^{143} \mathrm{Nd} /{ }^{144} \mathrm{Nd}\right)_{\text {sample }}-0.51315\right) /\left(\left({ }^{147} \mathrm{Sm} /{ }^{144} \mathrm{Nd}\right)_{\text {sample }}-0.2137\right)\right.$, where $\left({ }^{147} \mathrm{Sm} /{ }^{144} \mathrm{Nd}\right)_{\mathrm{CHUR}}=0.1967,\left({ }^{143} \mathrm{Nd} /{ }^{144} \mathrm{Nd}\right)_{\mathrm{CHUR}}=0.512638, \lambda_{\mathrm{Sm}}=6.54 \times 10-12 \mathrm{year}^{-1}$ (Lugmair and Marti, 1978); $\mathrm{T}_{\mathrm{DM} 2}=\mathrm{T}_{\mathrm{DM} 1}-\left(\mathrm{T}_{\mathrm{DM} 1}-\mathrm{t}\right) \times\left(f_{\mathrm{cc}}-f_{\text {sample }}\right) /\left(f_{\mathrm{cc}}-f_{\mathrm{DM}}\right)$, where $f_{\mathrm{cc}}=-0.4$, $f_{\mathrm{DM}}=0.0859$.

\section{Analytical results}

\subsection{Zircon $\mathrm{U}-\mathrm{Pb}$ geochronology}

Zircon grains selected from these six samples (TK61, TK84, TK85, TK164, TK216, TK264) are mostly euhedral to subhedral, transparent and colorless, ranging from $50 \mu \mathrm{m}$ to $210 \mu \mathrm{m}$ in length, and have length to width ratio between 1:1 to 3:1. Cathodoluminescence images show that representative zircons have commonly concentric oscillatory zoning with low to variable luminescence that indicates a magmatic origin (Fig. 5). The Th/U ratios of all samples are between 0.04 and 3.3, almost all $\mathrm{Th} / \mathrm{U}$ ratios are ranging from 0.1 to 1.0 , except a few zircons with low $\mathrm{Th} / \mathrm{U}$ ratios. $\mathrm{U}-\mathrm{Pb}$ data sets for all samples are given in Table 2. 

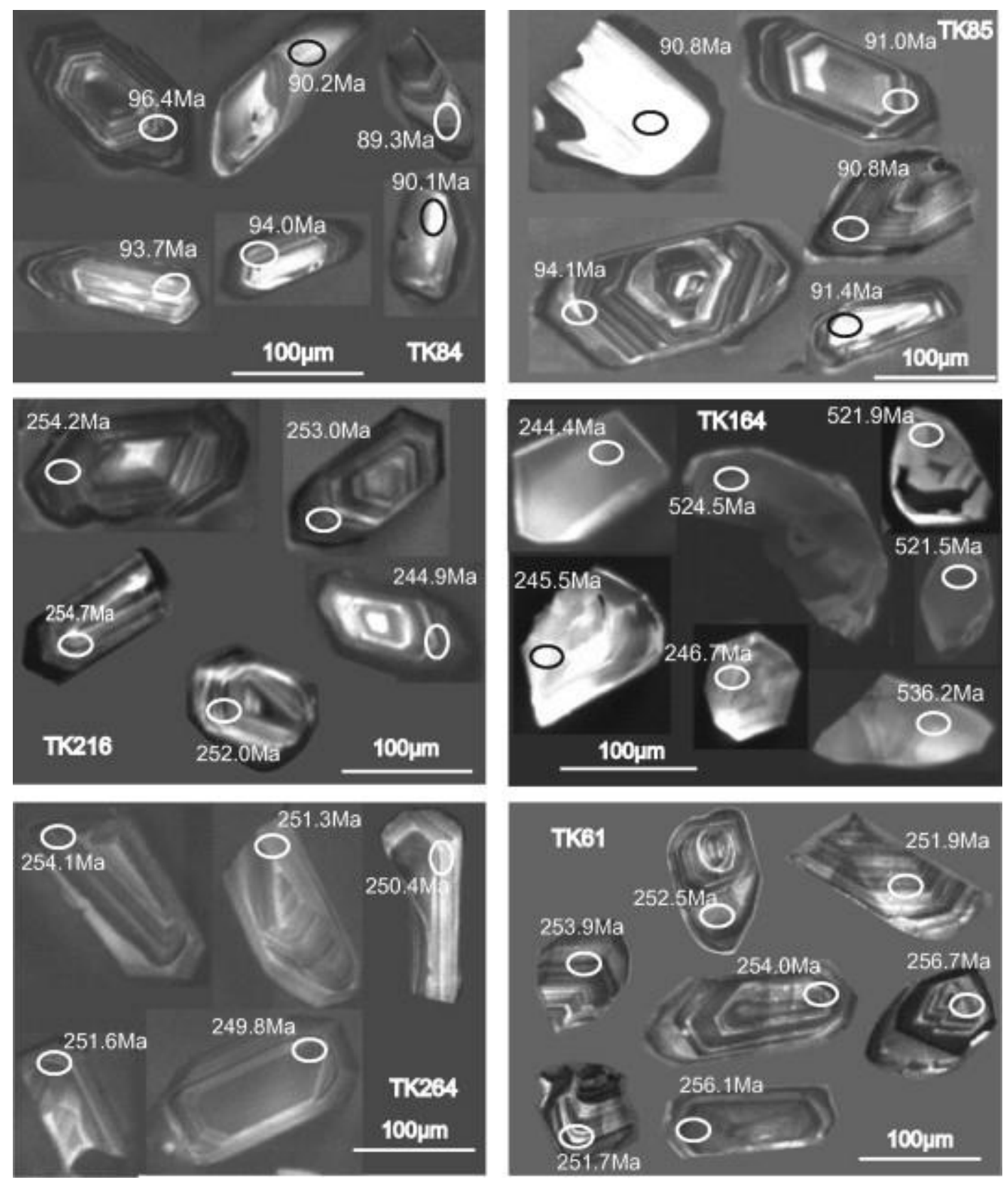

Fig. 5. : Cathodoluminescence images of representative zircons analyzed in situ for $\mathrm{U}-\mathrm{Pb}, \mathrm{Hf}$ and $\mathrm{O}$ isotopes. Small ellipses indicate the SIMS analysis spots for $\mathrm{U}-\mathrm{Pb}$ isotopes. Numbers near the analysis spots are the $\mathrm{U}-\mathrm{Pb}$ ages.

\subsubsection{Two-mica Leucogranites (TK84, TK85)}

Zircon grains in these samples are euhedral to subhedral, ranging from $50 \mu \mathrm{m}$ to $200 \mu \mathrm{m}$ in length, with length to width ratios between 1:1 and 3:1. Euhedral concentric zoning is common, and some grains have inherited cores (Fig. 5). Fifteen grains of sample TK84 were analyzed by SIMS, these analyses have variable concentrations of uranium (310-5692 ppm) and thorium (54-2327 ppm), with $\mathrm{Th} / \mathrm{U}$ ratios ranging from 0.042 to 3.314 , two $\mathrm{Th} / \mathrm{U}$ ratios are less than 0.1 . Three analyses give relatively older ages of $831 \mathrm{Ma}, 845 \mathrm{Ma}$ and $852 \mathrm{Ma}$, two analyzes yield ages of $513 \mathrm{Ma}$ and $418 \mathrm{Ma}$, these may be due to inherited grains, and three nearly concordant ages of ca.94 Ma. Seven analyses yield a concordia age of 
$90.1 \pm 1.0 \mathrm{Ma}$, which is interpreted as the crystallization age of the two mica leucogranite (Fig. 6a).
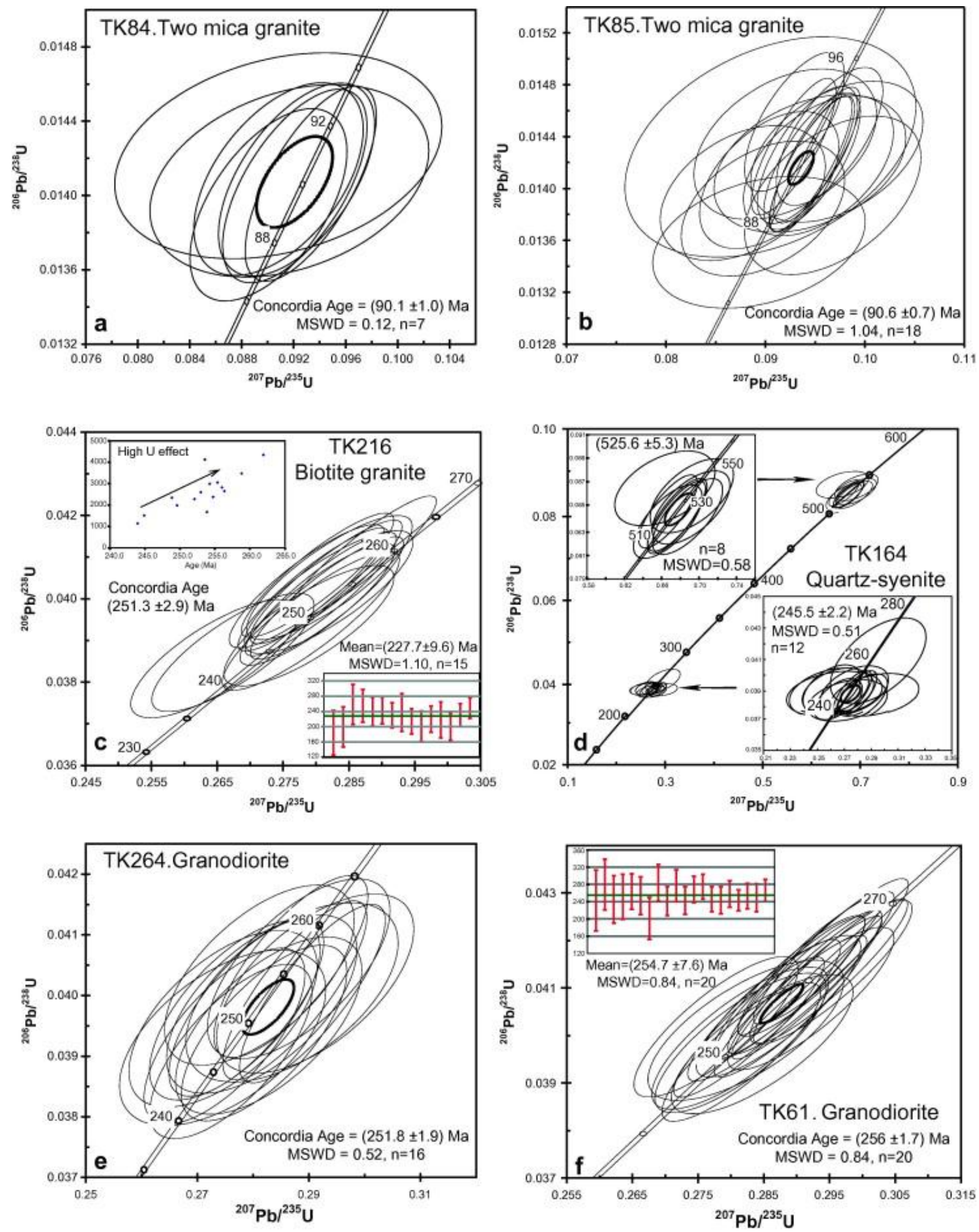

Fig. 6. : Conventional SIMS U-Pb zircon concordia diagram for the dated samples of northern Vietnam granitic rocks. 
Twenty grains of sample TK85 were analyzed by SIMS, uranium and thorium concentrations are as follows: $\mathrm{U}=142-4184 \mathrm{ppm}$ and $\mathrm{Th}=187-5318 \mathrm{ppm}$, with $\mathrm{Th} / \mathrm{U}$ ratios ranging from 0.09 to 2.41 , two $\mathrm{Th} / \mathrm{U}$ ratios are less than 0.1 . One grain gives a relatively old age at $818 \mathrm{Ma}$, while another one gives a nearly concordant age at 94.3 Ma. Eighteen analyses yield a concordia age of $90.6 \pm 0.7 \mathrm{Ma}$ (Fig. 6b). This age is consistent with the age of sample TK84 and is interpreted as the crystallization age of the two mica leucogranite.

\subsubsection{Biotite granite (TK216)}

Zircon grains in the TK216 sample are dominantly euhedral, ranging from $50 \mu \mathrm{m}$ to $150 \mu \mathrm{m}$ in length, with length to width ratios between 1:1 and 2:1. Concentric zoning is common, and some grains have inherited zircon cores (Fig. 5). Twenty-one grains were analyzed by SIMS, uranium and thorium concentrations are as follows: $U=1146-4346 \mathrm{ppm}$ and a major population of $\mathrm{Th}=294-2158 \mathrm{ppm}$, with $\mathrm{Th} / \mathrm{U}$ ratios ranging from 0.105 to 0.523 . Six analyses give relatively old ages and may be from the inherited zircons. Fifteen analyses yield a concordia age of $251.3 \pm 2.9 \mathrm{Ma}$ (Fig. 6c). But, these analyses show "high uranium effect", the ages become older while the uranium concentrations go higher ( $\mathrm{Li}$ et al., 2010a, Fig. 6e).

Thus, the realistic age maybe younger than this analyzed age. Considering that the ${ }^{207} \mathrm{~Pb} /{ }^{206} \mathrm{~Pb}$ ages are not affected by the high uranium concentration, the weighted mean ${ }^{207} \mathrm{~Pb} /{ }^{206} \mathrm{~Pb}$ age of $227.7 \pm 9.6 \mathrm{Ma}(\mathrm{MSWD}=1.1)$ (Fig. 6c) can be considered as the emplacement age of the pluton.

\subsubsection{Quartz-syenite (TK164)}

Zircon grains from this sample are subhedral, ranging from $50 \mu \mathrm{m}$ to $150 \mu \mathrm{m}$ in length, with length to width ratios between 1:1 and 1.5:1. Concentric oscillatory zoning is not clear, and most of the grains have inherited zircon cores (Fig. 5). Twenty analyses yield two groups of concordia age (Fig. 6d). One group is at $245.5 \pm 2.2 \mathrm{Ma}$; these analyses have variable concentrations of uranium (75-656 ppm) and thorium (37-614 ppm), with Th/U ratios ranging from 0.234 to 1.684 , which indicates a magmatic origin. Another group of zircons has a concordia age of $525.6 \pm 5.3 \mathrm{Ma}$. These analyses also have variable concentrations of uranium (105-462 ppm) and thorium (34-344 ppm), with Th/U ratios ranging from 0.234 to 0.773 , which also indicates their magmatic origin. The age of $245 \mathrm{Ma}$ is interpreted as the crystallization age of the quartz-syenite, and the age of $525 \mathrm{Ma}$ is interpreted as the age of inherited zircon.

\subsubsection{Granodiorite (TK264)}

Zircon grains from sample TK264 are euhedral to subhedral, ranging from $50 \mu \mathrm{m}$ to $200 \mu \mathrm{m}$ in length, with length to width ratios between 1:1 and 3:1. Euhedral concentric zoning is common, and some grains have inherited cores (Fig. 5). Twenty-two grains of sample TK264 were analyzed by SIMS, uranium and thorium concentrations are as follows: $U=396-$ $1779 \mathrm{ppm}$ and $\mathrm{Th}=142-742 \mathrm{ppm}$, with $\mathrm{Th} / \mathrm{U}$ ratios ranging from 0.142 to 0.546 . Two analyses give relatively old ages, which may be from inherited zircons and four analyses are nearly concordant. In the concordia diagram, sixteen analyses yield a concordia age of $251.8 \pm 1.9 \mathrm{Ma}$ (Fig. 6e). This age is interpreted as the crystallization age of the granodiorite. 


\subsubsection{Granodiorite (sample TK61)}

Zircon grains of the sample TK61 are mainly euhedral, ranging from $80 \mu \mathrm{m}$ to $120 \mu \mathrm{m}$ in length, with length to width ratios between $1: 1$ and 3:1. Euhedral concentric zoning is common and no inherited cores are observed (Fig. 5). Twenty three grains were analyzed by SIMS, uranium and thorium concentrations are as follows: $U=773-8198$ ppm with a major population between $2000 \mathrm{ppm}$ and $6000 \mathrm{ppm}$ and $\mathrm{Th}=87-6803 \mathrm{ppm}$ with a major population between $800 \mathrm{ppm}$ and $4000 \mathrm{ppm}$. The Th/U ratios range from 0.113 to 0.830 , with a maximum between 0.4 and 0.7 , which indicates a magmatic origin. Twenty analyses yield a concordia age of $256.9 \pm 1.7 \mathrm{Ma}$ (Fig. 6f). But these analyses have "high uranium effect", the ages become older while the uranium concentrations go higher (Li et al., 2010a). So the realistic age maybe younger than this measured age. Considering with the ${ }^{207} \mathrm{~Pb} /{ }^{206} \mathrm{~Pb}$ age are not affected by the uranium concentrations, the weighted mean ${ }^{207} \mathrm{~Pb} /{ }^{206} \mathrm{~Pb}$ age of $254.8 \pm 7.6 \mathrm{Ma}(\mathrm{MSWD}=0.84$, Fig. $6 \mathrm{f}$ ) is reliable and can be considered as age of granodiorite crystallization.

\subsection{Zircon Hafnium-0xygen isotope}

Zircons, which are highly retentive of the magmatic oxygen isotopes, in equilibrium with mantle-derived magmas have very consistent $\delta^{18} \mathrm{O}$ value of $5.3 \pm 0.3 \%$ (Valley et al., 1998 and Valley et al., 2005). This value is insensitive to magmatic differentiation, because the rise of $\delta^{18} \mathrm{O}$ in bulk rock is compensated by an increase in zircon liquid $\delta^{18} \mathrm{O}$ fractionation from $+0.5 \%$ for mafic melts to $+1.5 \%$ for silicic derivatives (Valley et al., 2005). Thus $\delta^{18} \mathrm{O}$ values in zircon above 5.6\%ofingerprint an ${ }^{18} \mathrm{O}$ enriched supracrustal component in the magma from which the zircon crystallized. This component is either sedimentary rock $(10-30 \%)$ or altered volcanic rock (up to 20\%o) (Eiler, 2001).

\subsubsection{Pia Oac pluton (Granites, samples TK84, TK85)}

Seven and sixteen in situ $\mathrm{Hf}$ and $\mathrm{O}$ isotopic analyses were carried out for zircons from the two mica leucogranite samples TK84 and Tk85, respectively (Table 3). The zircons from sample TK84 have a wide range of oxygen isotopic compositions, with $\delta^{18} \mathrm{O}=8.19 \%{ }_{0}-9.87 \%$ (Fig. 7a). Similarly, the zircon from sample TK 85 also have a wide range of oxygen isotopic compositions, with $\delta^{18} \mathrm{O}=8.12 \%{ }^{0}-10.95 \%$ (Fig. $7 \mathrm{~b}$ ). For the sample TK84, the value of ${ }^{176} \mathrm{Hf} /{ }^{177} \mathrm{Hf}$ range from 0.282424 to 0.282643 , the calculated $\varepsilon_{\mathrm{Hf}}(t)$ vary from -10.4 to -2.7 and the $\mathrm{T}_{\mathrm{DM}}{ }_{\mathrm{C}}$ ages vary from $1330 \mathrm{Ma}$ to $1744 \mathrm{Ma}$. In the sample TK85, the value of ${ }^{176} \mathrm{Hf} /{ }^{177} \mathrm{Hf}$ range from 0.282383 to 0.282564 , the calculated $\varepsilon_{\mathrm{Hf}}(t)$ vary from -11.8 to -5.5 and the $\mathrm{T}_{\mathrm{DM}}{ }^{\mathrm{C}}$ ages vary from $1903 \mathrm{Ma}$ to $1508 \mathrm{Ma}$. 

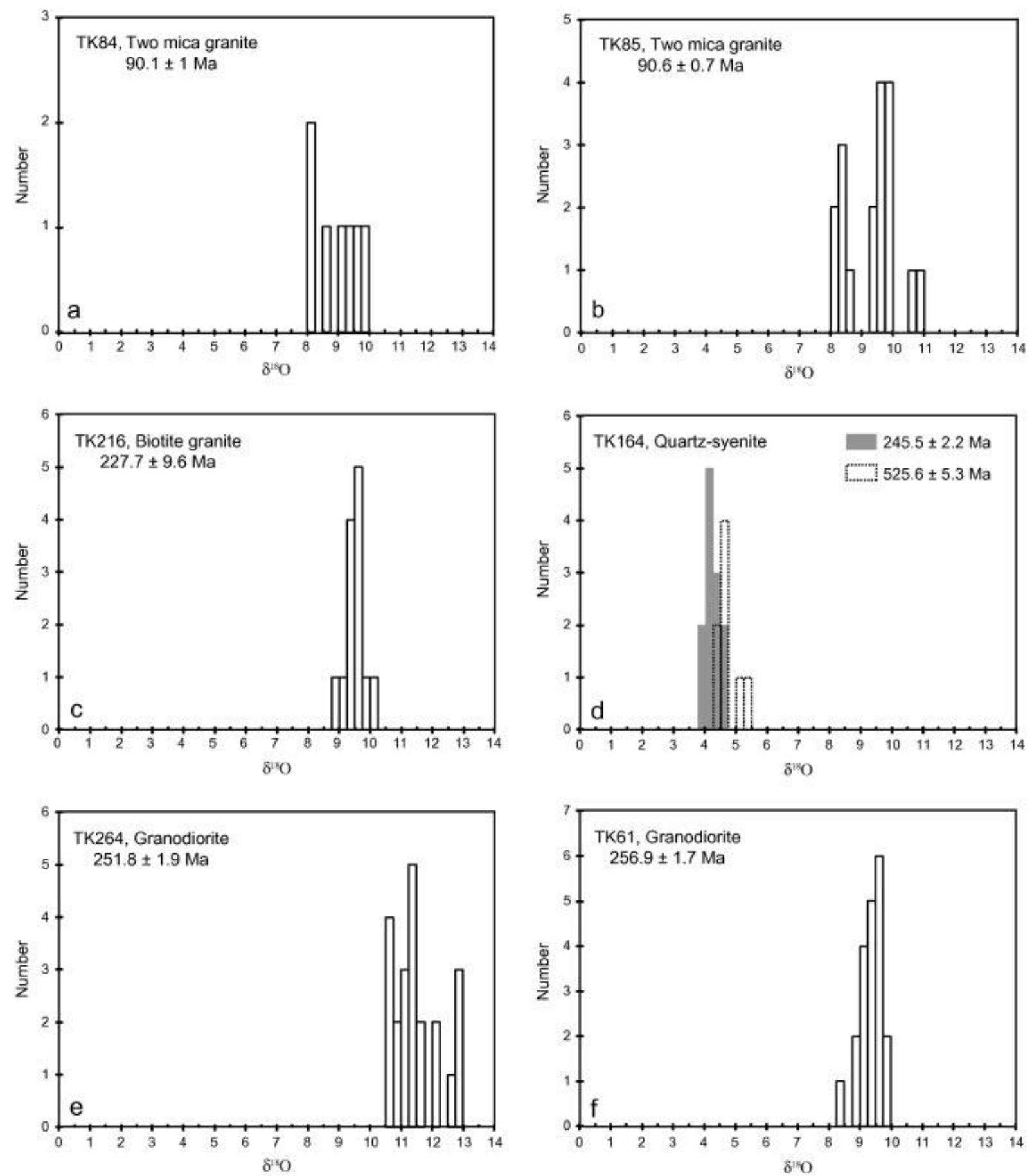

Fig. 7. : Histogram of $\delta^{18} \mathrm{O}$ values for the analyzed northeastern Vietnam granitic rocks.

\subsubsection{Bach Ha pluton (Biotite granite, sample TK216)}

Thirteen in situ Hf and $\mathrm{O}$ isotopic analyses were carried out for zircons from the sample (TK216). The zircon have a relatively small range of $\mathrm{Hf}$ and $\mathrm{O}$ isotopes:

${ }^{176} \mathrm{Hf} /{ }^{177} \mathrm{Hf}=0.282362-0.282453, \varepsilon_{\mathrm{Hf}}(t)$ vary from -9.4 to -6.0 , the calculated $\mathrm{T}_{\mathrm{DM}}{ }^{\mathrm{C}}$ ages vary from $1869 \mathrm{Ma}$ to $1666 \mathrm{Ma}$, and the value of $\delta^{18} \mathrm{O}$ range from $8.94 \%$ o to $10.23 \%$ (Fig. $7 \mathrm{c})$. 


\subsubsection{Pia Ma pluton (Quartz-syenite, sample TK164)}

Twenty in situ $\mathrm{Hf}$ and $\mathrm{O}$ isotopic analyses were carried out for zircons from the sample (TK164). Considering that this sample yields two concordia zircon SIMS U-Pb ages, the zircons from this sample can be classified into two groups. One group of zircons is ca. $245 \mathrm{Ma}$, while another group is ca. $525 \mathrm{Ma}$. Both of two groups of zircons get quite consistent $\mathrm{O}$ isotopic character. The value of $\delta^{18} \mathrm{O}$ ranges from $3.99 \%$ to $4.74 \%$ (Fig. $7 \mathrm{~d}$ ) in the former group (ca. $245 \mathrm{Ma}$ ). The later group with the value of $\delta^{18} \mathrm{O}$ ranges from $4.32 \%$ to $5.26 \%$ (Fig. $7 \mathrm{~d}$, Table 3). For the former group of zircons: ${ }^{176} \mathrm{Hf} /{ }^{177} \mathrm{Hf}=0.282428-0.282576$, corresponding to $\varepsilon_{\mathrm{Hf}}(t)=-6.8-(-0.2)$, and the calculated $\mathrm{T}_{\mathrm{DM}}{ }_{\mathrm{C}}$ ages vary from $1712 \mathrm{Ma}$ to $1289 \mathrm{Ma}$. The later group has the Hf isotopic character as follows: ${ }^{176} \mathrm{Hf} /{ }^{177} \mathrm{Hf}=0.282452$ 0.282546 , corresponding to $\varepsilon_{\mathrm{Hf}}(t)=0.2-3.1$, and the calculated $\mathrm{T}_{\mathrm{DM}}{ }^{\mathrm{C}}$ ages vary from $1490 \mathrm{Ma}$ to $1294 \mathrm{Ma}$.

\subsubsection{Diem Mac pluton (Granodiorite, sample TK264)}

Twenty in situ $\mathrm{Hf}$ and $\mathrm{O}$ isotopic analyses were carried out for zircons from the sample (TK264). These zircons have a relatively small range of Hf isotopes: ${ }^{176} \mathrm{Hf} /{ }^{177} \mathrm{Hf}=0.282337-$ 0.282458 . The values of $\varepsilon_{\mathrm{Hf}}(t)$ vary from -10.0 to -7.0 , the calculated $\mathrm{T}_{\mathrm{DM}}{ }^{\mathrm{C}}$ ages vary from $1915 \mathrm{Ma}$ to $1644 \mathrm{Ma}$, and the value of $\delta^{18} \mathrm{O}$ range from $10.61 \%$ to $12.81 \%$ o (Fig. $7 \mathrm{e}$ ).

\subsubsection{Cǒ Linh pluton (Granodiorite, sample TK61)}

Twenty-three in situ $\mathrm{Hf}$ and $\mathrm{O}$ isotopic analyses were carried out for zircons from the sample (TK61), and the data are listed in Table 3. The zircon have a relatively small range of $\mathrm{Hf}$ and $\mathrm{O}$ isotopes: ${ }^{176} \mathrm{Hf} /{ }^{177} \mathrm{Hf}=0.282473-0.282566$, corresponding to $\varepsilon_{\mathrm{Hf}}(t)=-5.2-(-2.1)$, the calculated $\mathrm{T}_{\mathrm{DM}}{ }^{\mathrm{C}}$ ages vary from $1618 \mathrm{Ma}$ to $1417 \mathrm{Ma}$, and $\delta^{18} \mathrm{O}=8.46-9.95 \%$ (Fig. $7 \mathrm{f}$ ).

\subsection{Bulk-rock Sr-Nd isotope data}

The initial ${ }^{87} \mathrm{Sr} /{ }^{86} \mathrm{Sr}\left(\mathrm{I}_{\mathrm{Sr}}\right)$ ratios and $\varepsilon_{\mathrm{Nd}}(t)$ values were calculated using the U-Pb zircon ages determined in this study (Table 4). We tested one sample from Late Permian to Early Triassic granodiorite (TK61) and two samples from Late Cretaceous granite (TK84, TK85). The depleted mantle model ages $\left(\mathrm{T}_{\mathrm{DM}}\right)$ are reported using the model of De Paolo (1981). The two stages depleted mantle model ages $\left(\mathrm{T}_{\mathrm{DM} 2}\right)$ are used to reduce the error of the $\mathrm{T}_{\mathrm{DM}}$, which are caused by the $\mathrm{Sm}$ and $\mathrm{Nd}$ fractionation during the process of crustal partial remelting ( Liew and Hofmann, 1988 and Li, 1996). The Có Linh granodiorite (Sample TK61) yields ${ }^{87} \mathrm{Rb} /{ }^{86} \mathrm{Sr}$ and ${ }^{87} \mathrm{Sr} /{ }^{86} \mathrm{Sr}$ values of 1.61 and 0.716363 , respectively. The age-corrected ${ }^{87} \mathrm{Sr} /{ }^{86} \mathrm{Sr}$ ratio is 0.7105 . This rock displays ${ }^{147} \mathrm{Sm} /{ }^{144} \mathrm{Nd}$ ratio 0.0947 and the measured ${ }^{143} \mathrm{Nd} /{ }^{144} \mathrm{Nd}$ ratio 0.511959. Calculated initial $\varepsilon_{\mathrm{Nd}}(t)$ value is -9.7 . The $\mathrm{T}_{\mathrm{DM}}$ and $\mathrm{T}_{\mathrm{DM} 2}$ ages of sample TK61 are $1.52 \mathrm{Ga}$ and $1.87 \mathrm{Ga}$, respectively. The two mica leucogranite (samples TK84 and TK85) from the Pia Oac pluton has quite similar $\mathrm{Sr}-\mathrm{Nd}$ isotopic characteristics. The measured ${ }^{87} \mathrm{Rb} /{ }^{86} \mathrm{Sr}$ values of samples TK84 and TK85 are 49.5 and 47.0 while ${ }^{87} \mathrm{Sr} /{ }^{86} \mathrm{Sr}$ values are 0.788359 and 0.788476 , respectively. The age-corrected ${ }^{87} \mathrm{Sr} /{ }^{86} \mathrm{Sr}$ ratios of these two samples are 0.7251 and 0.7283 , respectively. These two samples display ${ }^{147} \mathrm{Sm} /{ }^{144} \mathrm{Nd}$ ratio 0.1333 and 0.1370 while the measured ${ }^{143} \mathrm{Nd} /{ }^{144} \mathrm{Nd}$ ratio are 0.512034 and 0.512061 . Calculated initial $\varepsilon_{\mathrm{Nd}}(t)$ values are -11 and -10.5 , respectively. The $\mathrm{T}_{\mathrm{DM}}$ ages of samples TK84 and TK85 are $2.11 \mathrm{Ga}$ and $2.15 \mathrm{Ga}$ while the $\mathrm{T}_{\mathrm{DM} 2}$ ages of these two samples are $1.79 \mathrm{Ga}$ and $1.75 \mathrm{Ga}$, respectively. 


\section{Discussion}

\subsection{Material source of NE Vietnam granitic rocks}

The granitic rocks of NE Vietnam have high negative $\varepsilon_{\mathrm{Nd}}(t)$ values, which range from -11 to -9.7 , and higher ${ }^{87} \mathrm{Sr} /{ }^{86} \mathrm{Sr}(0.7105-0.7283)$ ratios indicating crustal source (Table 4). The crustal residence age $\mathrm{T}_{\mathrm{DM} 2}$ values range from $1.87 \mathrm{Ga}$ to $1.75 \mathrm{Ga}$. The characteristics of $\mathrm{Nd}$ isotope are similar to those of the Proterozoic basement of SCB ( Li, 1994 and Shen et al., 1998). This suggests that ancient continental crust material was involved in the granitic rocks genesis. Almost all of the $\delta^{18} \mathrm{O}$ values range from $8 \%$ to $12 \%$, except the Pia Ma pluton with $\delta^{18} \mathrm{O}$ values ranging from $4.0 \%$ to $5.3 \%$ (Fig. $7 \mathrm{~d}$ ). The similarity of their geochemical features implies that they were derived from similar sources, with various degrees of differentiation. Furthermore, taking into account the zircon Lu-Hf isotope, the $\varepsilon_{\mathrm{Hf}}(t)$ values of zircon from the northeastern Vietnam vary from -11.8 to 3.1, implying a minor input of mantle-derived materials during the magma generation, but mostly crustal involvement. The calculated $\mathrm{T}_{\mathrm{DM}}{ }^{\mathrm{C}}$ ages vary from $1.93 \mathrm{Ga}$ to $1.33 \mathrm{Ga}$, this result is consistent with the $\mathrm{Nd}$ isotopic $\mathrm{T}_{\mathrm{DM} 2}$ ages. The TK164 sample from the Pia Ma quartz-syenite pluton shows two different isotopic characters corresponding to two groups in age (Fig. 6). The young group has negative $\varepsilon_{\mathrm{Hf}}(t)$ values that range from -6.8 to -0.2 , and has a lower $\delta^{18} \mathrm{O}$ value ranging from $3.99 \%$ o to $4.74 \%$. The old group has positive $\varepsilon_{\mathrm{Hf}}(t)$ with values ranging from 0.2 to 3.1 . This rock also exhibits high $\delta^{18} \mathrm{O}$ values ranging from $4.32 \%$ to $5.26 \%$. Considering these isotopic features, we argue that this quartz-syenite originated from juvenile mantle magma during the formation and growth of continental crust then was re-melted by a young stage of magmatism. Thus our geochemical data suggest that the granitic rocks from the NE Vietnam were derived from the melting of a Proterozoic basement similar to that of the SCB (Fig. 8).

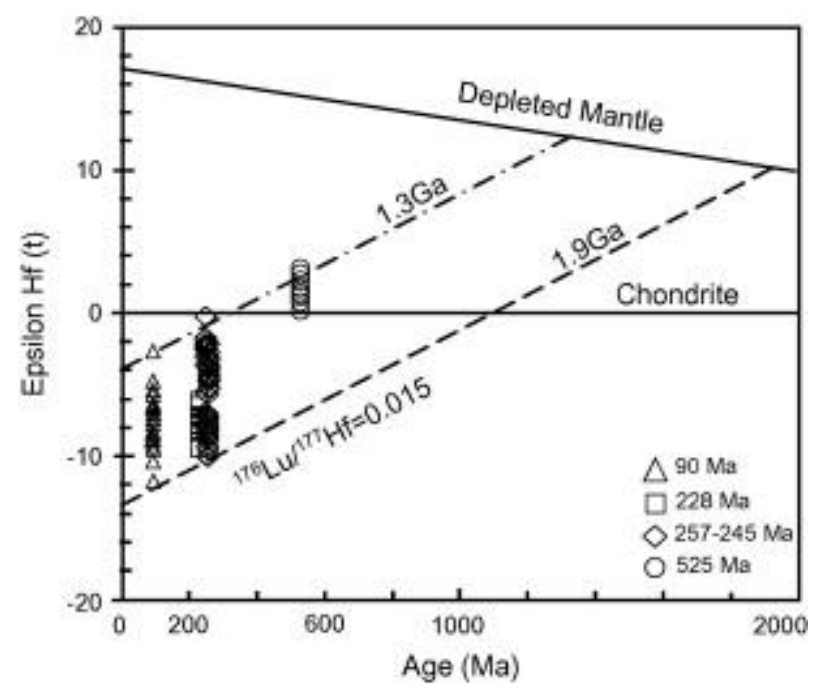

Fig. 8. : Diagram of zircon $\varepsilon_{\mathrm{Hf}}(t)$ isotopic composition versus ages of all zircons from northeastern Vietnam granitic rocks. 


\subsection{Magmatism of NE Vietnam and related tectonic significance}

\subsubsection{Early Paleozoic magmatism}

This magmatism is well exposed in the southeast part of the SCB (Fig. 1). It is mostly distributed in the Wuyi-Baiyun-Yunkai areas (Lin et al., 2008 and reference therein). The Early Paleozoic age is also supported by the regional geology, since unmetamorphosed Late Devonian and younger sediments unconformably cover the pre-Devonian plutons and their low-grade metasedimentary host rocks (HBGMR, 1988 and JBGMR, 1984). In the present state of knowledge, the detail tectonics of the emplacement setting of these plutons is not settled yet. A crustal-thickening model was proposed because of the granites are of crustal origin (Faure et al., 2009, Charvet et al., 2010, Li et al., 2010c and Wang et al., 2011). A postthickening extensional setting was suggested on the basis of structural observation in the Yunkai massif (Lin et al., 2008). In northeastern Vietnam, the Song Chay porphyritic granite, dated at 430-420 Ma (Fig. 2; Roger et al., 2000 and Carter et al., 2001), emplaced at the end of the Early Paleozoic orogeny. However, the intense foliation developed during the Triassic orogeny precludes any study of the syn-magmatic structures.

In NE Vietnam, as mentioned before, the quartz syenite sample (TK164) from the Pia Ma pluton yields two groups of zircon age (Fig. 3 and Fig. 6). The old group (eight grains) yields a 525.6 $\pm 5.3 \mathrm{Ma}$ age (Fig. 6). The isotopic characteristics (Table 3) show that these grains are inherited from a juvenile mantle-derived magma during the formation of the continental crust (Fig. 7 and Fig. 8). In the SCB, such Early Paleozoic ages (ca. 530-500 Ma) are quite rare. Song et al. (2007) reported Early Paleozoic granites (520-480 Ma) in NW Yunnan province. Also, the $537 \mathrm{Ma}$ to $507 \mathrm{Ma}$ ages found in the Guzhai pluton in eastern Guangdong province are interpreted as those of a magmatic event (Ding et al., 2005). Several zircon grains with ages around $537-507 \mathrm{Ma}$ are obtained in the Yunkai massif in granites yielding ${ }^{206} \mathrm{~Pb} /{ }^{238} \mathrm{U}$ apparent ages $421.9 \pm 9.8 \mathrm{Ma}$ to $440.7 \pm 5.6 \mathrm{Ma}$ (Wang et al., 2007c). Chen et al. (2009a) argued for the existence of a magmatic or thermal event existent in SCB during the Cambrian time (at ca. $526 \mathrm{Ma}$ ). Even if we cannot completely rule out the possibility of a mixed age, our CL images support the existence of magmatic zircon around this period (Fig. 5). The significance of this magmatism is unknown. It might be related to the rifting stage experienced by the SCB from Neoproterozoic to Cambrian times (JBGMR, 1984, HBGMR, 1988 and Wang et al., 2010).

\subsubsection{Late Permian-Early Triassic magmatism}

In the same TK164 sample, a second zircon group yields an age around $245.5 \pm 2.2 \mathrm{Ma}$. The isotopic analyses show that these zircons are due to a re-melting of the continental crust and a limited input by mantle material (Table 4; Fig. 7 and Fig. 8). From the regional geology of northeastern Vietnam, at least two magmatic episodes can be distinguished during the Triassic (Indosinian) orogeny (Tran et al., 2008 and Liu et al., 2012; Roger et al., 2012 and references therein). The earliest plutons were emplaced at ca. 280-240 Ma (Late Permian to Early Triassic), while the latest ones were emplaced at ca. 230-200 Ma (Late Triassic). Our geochronological results from samples TK61, TK164, TK264 relate to the first magmatic episode that appears as concentrated in a narrow time span, from $257 \mathrm{Ma}$ to $245 \mathrm{Ma}$, with two different groups of the $\delta^{18} \mathrm{O}$ value range from $3.99 \%$ to $4.74 \%$ ond $8.4 \%$ o to $12.8 \%$, respectively (Fig. 6 and Fig. 7; Table 3). The zircon Hf isotopic analyses indicate two groups of negative $\varepsilon_{\mathrm{Hf}}(t)$ value range from -10.0 to -7.0 and -5.0 to -2.0 , respectively (Fig. 8; Table 3 ). The $\varepsilon_{\mathrm{Nd}}(t)$ value is -9.7 and ${ }^{87} \mathrm{Sr} /{ }^{86} \mathrm{Sr}$ ratio is 0.71055 (Table 4). In the NE Vietnam, 
gabbro-syenite, gabbro-monzodiorite, basalt-ryholite associations and high-Al granitic rocks dated of Late Permian to Early Triassic by zircon U-Pb and biotite or amphibole ${ }^{40} \mathrm{Ar} /{ }^{39} \mathrm{Ar}$ geochronology (Fig. 3; Tran et al., 2008). The Permian alkaline magmatism is interpreted as the result of the Emeishan mantle plume ( Chung and Jahn, 1995, Hanski et al., 2004, Xu et al., 2001, Xu et al., 2004 and Tran et al., 2008). The Late Permian to Early Triassic granites are also recognized in the southwestern part of the SCB (Deng et al., 2004, Ding et al., 2005, Xie et al., 2006 and Chen et al., 2011; Fig. 1).

\subsubsection{Late Triassic Magmatism}

In northeastern Vietnam, the Late Triassic magmatism is rarely reported (Fig. 3). Because of the "high uranium effect" (Li et al., 2010a), the TK216 sample of the undeformed biotitic granite yields ${ }^{207} \mathrm{~Pb} /{ }^{206} \mathrm{~Pb}$ age of $227.7 \pm 9.6 \mathrm{Ma}$ (Fig. 6). According to our analyses, the isotopic features of these granitic rocks show negative $\varepsilon_{\mathrm{Hf}}(t)$ with the values ranging from -9.0 to -6.0 , and $\delta^{18} \mathrm{O}$ values ranging from $8.94 \%$ to $10.23 \%$. These data indicate the magma originated from a crustal source ( Fig. 7 and Fig. 8).

In SCB, Late Triassic granitic plutons are widespread but they are related to different tectonic settings depending on their location (Fig. 1; Deng et al., 2004, Wang et al., 2007b and Chu et al., 2012). The Late Triassic granites exhibit an affinity to both S-type and I-type petrology (Wang et al., 2007b). Their zircon $\varepsilon_{\mathrm{Hf}}(t)$ values range from -2.0 to -20.2 with $\mathrm{Hf}$ model ages of 1.2 to $2.5 \mathrm{Ga}$ (Qi et al., 2007). In the Xuefengshan intracontinental belt, in the central part of the SCB, the magmatism was interpreted as a late- to post-orogenic partial melting (Chu et al., 2012). Although these plutons were related to extensional tectonics ( Zhou et al., 2006, Wang et al., 2012b and Li et al., 2012), presently there is not structural data that support this view. In the northern part of the SCB, the Middle Triassic tectonics was interpreted as an extensional one developed in the southern foreland of the Dabieshan ( Faure et al., 1996 and Faure et al., 1999). Triassic plutons are scattered in the SE part of the SCB, but their geodynamic significance remains unclear. In NE Vietnam, the Triassic orogeny is represented by N- to NE-vergent folds and thrust (Lepvrier et al., 2011). This orogeny is also responsible for the development of a pervasive post-solidus foliation in the Early Paleozoic plutons. The NE Vietnam Late Triassic plutons developed at the expense of a thickened continental crust. Therefore, we argue that these plutons are post-collisional bodies.

In northeastern Vietnam, the ${ }^{40} \mathrm{Ar}-{ }^{39} \mathrm{Ar}$ geochronological data of synkinematic minerals give a large span of ages from $250 \mathrm{Ma}$ to $198 \mathrm{Ma}$ (Fig. 9) that are interpreted as deformation age. But on the basis of our field observations, the Mesozoic deformation more likely occurred in the late Middle Triassic or early Late Triassic because the middle Triassic sedimentary succession is involved in the deformation. In the northern part of the Song Chay massif, the metamorphic rims of the igneous zircon grains yield an average ${ }^{206} \mathrm{~Pb} /{ }^{238} \mathrm{U}$ age of $237 \pm 15 \mathrm{Ma}$, and amphibole from a mylonitic rock yields a ${ }^{40} \mathrm{Ar} /{ }^{39} \mathrm{Ar}$ plateau age of $237 \pm 4.6$ Ma suggest that the main deformation occurred at ca.237 Ma (Yan et al., 2006). This age is consistent with the muscovite ${ }^{40} \mathrm{Ar} /{ }^{39} \mathrm{Ar}$ age of $236 \pm 0.5 \mathrm{Ma}$ from orthogneiss in the southern margin of the Song Chay massif (Maluski et al., 2001). Taking these works into account, we argue the deformation time ranges between $228 \mathrm{Ma}$ and $237 \mathrm{Ma}$. Our undeformed biotitic granite, which yields a zircon SIMS U-Pb age of 227.7 $\pm 9.6 \mathrm{Ma}$, supports this conclusion (Fig. 9). 


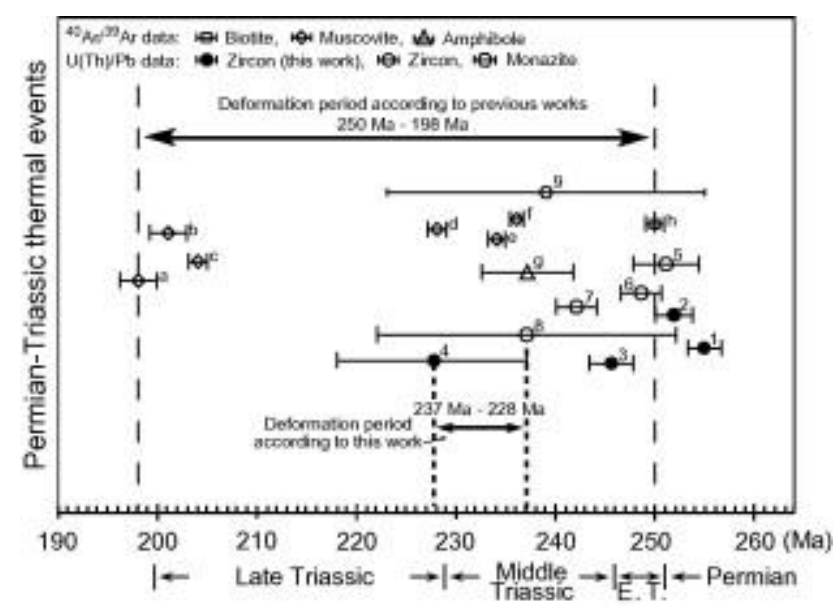

Fig. 9. : Diagram of geochronological data shows Permian-Triassic thermal events in the northeastern Vietnam. U(Th)/Pb data: 1. Granodiorite (Sample TK61); 2. Granodiorite (Sample TK264); 3. Quartz-syenite (Sample TK164); 4. Biotite granite (Sample TK216); 5. Gabbronorite (Tran et al., 2008); 6-7. Granite (Roger et al., 2012), 8. Igneous rock (Yan et al., 2006); 9. Garnet micaschist (Gilley et al., 2003). ${ }^{40} \mathrm{Ar} /{ }^{39} \mathrm{Ar}$ data: a. Marble (Maluski et al., 2001); b. Gneiss (Maluski et al., 2001); c. Micaschist (Maluski et al., 2001); d. Orthogneiss (Maluski et al., 2001); e. Micaschist (Maluski et al., 2001); f. Orthogneiss (Maluski et al., 2001); g. Mylonitic rock (Yan et al., 2006).

In the northern part of our study area, two granodiorite samples of TK61 and TK264 are dated at $255 \mathrm{Ma}$ and $251 \mathrm{Ma}$, respectively. However, these rocks exhibit no or weakly developed foliation. The lack of any pervasive ductile deformation in relatively ancient granodiorite can be explained in several ways. Firstly, these plutons are located rather far away from the main syn-metamorphic thrust zone, and thus escaped the ductile deformation (Fig. 2). Secondly, these rocks contain a majority of hard-strain minerals such as hornblende and K-feldspar, therefore these plutons could be deformed with great difficulty under moderate temperature conditions (Fig. 4). Thirdly, due to the limited outcrop conditions it can be argued that our sampling sites are located in undeformed cores of these plutons whereas their rims would be more foliated. Lastly, it can also be argued that there is a very short-lived tectonic stagnancy in this period although this possibility needs further detail structural work to be tested. Along the southern margin of the SCB, the top to the N/NE deformation is well documented both in Song Chay and Yunkai massifs (Lin et al., 2008 and Lepvrier et al., 2011). The Triassic deformation ages also cluster between $250 \mathrm{Ma}$ and $200 \mathrm{Ma}$ (Wang et al., 2005, Wang et al., 2007a, Cai and Zhang, 2009 and Zhang et al., 2011). The central part of the Yunkai massif, and its northern thrust zone, yield metamorphic minerals dated at 230-208 Ma by the ${ }^{40} \mathrm{Ar}-$ ${ }^{39} \mathrm{Ar}$ method (Wang et al., 2007a). This deformation phase is considered as the main ductile one in the Yunkai massif (Lin et al., 2008). At the regional scale, a similar ductile deformation has been recognized in the Song Chay orthogneiss, and its metamorphic country rocks (Roger et al., 2000, Maluski et al., 2001, Yan et al., 2006 and Lepvrier et al., 2011). According to Lepvrier et al. (2011), the SCB subducted to the South beneath the Day Nui Con Voi massif along the Song Chay suture during the Late Permian to Middle Triassic time. The top-to-the-NE/N deformation along the south margin of SCB is interpreted as the thrusting of the continental crust of the subducted plate. 


\subsubsection{The Late Cretaceous magmatism (ca. 95-85 Ma)}

In NE Vietnam, the granitic samples TK84 and TK85, dated around $90 \mathrm{Ma}$, have high $\mathrm{SiO}_{2}$ value (72.02-74.31\%), and the A/CNK value varies from 1.16 to 1.45 (Wang et al., 2011; our unpublished data). The initial ${ }^{87} \mathrm{Sr} /{ }^{86} \mathrm{Sr}$ ratio ranges from 0.7251 to 0.7283 , the value of $\delta^{18} \mathrm{O}$ ranges from $8.17 \%$ to $10.95 \%$ and the values of $\varepsilon_{\mathrm{Hf}}(t)$ vary from -11.7 to -2.7 ( Fig. 7 and Fig. 8). These geochemical features show that the Late Cretaceous granitic plutons of NE Vietnam are derived from a crustal source. The Late Cretaceous granitic rocks are also distributed in the southwestern and southeastern parts of the SCB (Fig. 1). The geochemical features of the Late Cretaceous granites are reported as high-K calc-alkaline I-type granites, as well as $A$-type granites, highly differentiated I- type granites and I- and A-type complexes ( Dong and Peng, 1994 and Martin et al., 1994; Chen and Jahn, 1998, Chen et al., 2000, Zhou et al., 2006, Qiu et al., 2008 and He et al., 2011). The magmatism occurred in the back-arc extensional tectonic setting ( Li, 2000, Jiang et al., 2011 and He et al., 2011). During the Late Cretaceous, the eastern part of SCB underwent lithospheric thinning coeval with an asthenospheric mantle upwelling that triggered mafic melt underplating ( $\mathrm{Li}, 2000$ and Yan et al., 2005). The geochemical features of the Late Cretaceous granites of NE Vietnam are similar to the Late Cretaceous S-type granite reported in southwest China (Cheng and Mao, 2010; and references therein). We suggest that the granitic rocks of this period in NE Vietnam were derived from the melting of continental crust.

Our geochronological and isotopic data show that the characteristics of magmatism in NE Vietnam are similar to South China block, especially in the southern margin of SCB. The stratigraphic sequence and bulk architecture of northeastern Vietnam and Chinese Guangxi provinces are comparable (Fig. 2; Table 1; Lepvrier et al., 2011). Furthermore, the PermoTriassic kinematics in NE Vietnam belt is also comparable to the Yunkai massif in the southern margin of SCB (Lin et al., 2008 and Lepvrier et al., 2011). Therefore, we argue that northeastern Vietnam is geologically part of the South China block. In the south margin of SCB (such as Chinese Yunnan and Guangxi provinces) and NE Vietnam, the gabbro, pillow basalts, mafic dykes cropping out have been considered as the "Babu ophiolites" (Cai and Zhang, 2009). The observations near Cao Bang show that the mafic rocks are olistoliths reworked in the Middle Triassic turbidite, as observed in Guangxi province, the gabbro intrudes the Late Paleozoic host rocks with a thermal metamorphism (Faure et al., submitted for publication). Thus these rocks did not form an ophiolitic suite but rather were emplaced in an intraplate setting related to the Emeishan plume (Tran et al., 2008). Though still speculated, an ophiolitic suture between NE Vietnam and South China appears unlikely there, as the suture zone is located along the Song Chay mélange.

\section{Conclusion}

The application of zircon $\mathrm{U}-\mathrm{Pb}$ geochronology and isotopic investigation, allow us to draw the following conclusions:

Zircon SIMS dating indicates that at least four thermal events occurred in the NE Vietnam: at ca. $525 \mathrm{Ma}$, at ca.254 Ma to $251 \mathrm{Ma}$, at ca.245-220 Ma, and at ca.91 Ma. The Early Paleozoic $(525 \mathrm{Ma})$ thermal event has positive $\varepsilon_{\mathrm{Hf}}(t)$ values ranging from 0.2 to 3.1 and $\delta^{18} \mathrm{O}$ value ranging from $4.32 \%$ to $5.26 \%$. This event might be related to the rifting experienced by the $\mathrm{SCB}$ in Neoproterozoic to Cambrian periods. 
The Late Permian to Early Triassic (254 Ma to $245 \mathrm{Ma}$ ) granitoids show zircon $\varepsilon_{\mathrm{Hf}}(t)$ ranging from -10 to -2.0 , and $\delta^{18} \mathrm{O}$ value ranging from $8.46 \%$ to $12.81 \%$. These features indicate crustal source for the granitic magma and limited input by mantle material. This magmatism is possibly related to the intraplate Emeishan magmatism. However, a regional extensional setting in the southern part of SCB cannot be discarded.

The Late Triassic (230-200 Ma) granitic rocks have negative $\varepsilon_{\mathrm{Hf}}(t)$ values ranging from -9.0 to -6.4 , and $\delta^{18} \mathrm{O}$ value ranging from $8.94 \%$ o to $10.23 \%$. The isotopic characters show these granitic rocks are also derived from a crustal source and represent post-orogenic plutons.

The Late Cretaceous granites (ca.90 Ma) are $S$-type granites and are derived from the melting of continental crust.

Our $\mathrm{Sr}-\mathrm{Nd}-\mathrm{Hf}-\mathrm{O}$ isotopic data suggest that the NE Vietnam granitoid magmas formed by partial melting of a Paleoproterozoic crust with minor input from mantle-derived material. Because of the poor outcrop and small size of these granitoid plutons, systematic geochemical works are still rare. Further works of integrated geochemistry and detail structural analyses are needed in order to substantiate the geological evolution of this part of the SCB.

\section{Acknowledgements}

Field and laboratory expenses have been funded by National Natural Science Foundation of China (41225009), Chinese National 973 Project (2009CB825008), the Innovative Project of the Chinese Academy of Sciences (Grant No. KZCX1-YW-15-1), and the Major National Science and Technology Project (No. 2011ZX05008-001). We thank Saihong Yang for help with zircon CL imaging, Qiuli Li, Wenbin Ji, Guoqiang Tang and Yu Liu for help with zircon $\mathrm{U} / \mathrm{Pb}$ analysis, and Yue-Heng Yang for help with zircon Hf analysis.

\section{References}

Bourret, 1922

R. Bourret

Les Nappes dans le Nord-Est du Tonkin. Comptes Rendus de l'Académie des Sciences - Series IIA

Earth and Planetary Science, 174 (1922), pp. 406-408

Cai and Zhang, 2009

J.X. Cai, K.J. Zhang

A new model for the Indochina and South China collision during the late permian to the middle triassic original research article

Tectonophysics, 467 (2009), pp. 35-43

Cai et al., 2006

M.H. Cai, L.Q. He, G.Q. Liu

SHRIMP zircon $\mathrm{U}-\mathrm{Pb}$ dating of the intrusive rocks in the Dachang tinpolymetallic ore district, Guangxi and their geological significance

Geological Review, 52 (2006), pp. 409-414 (in Chinese with English abstract)

Carter et al., 2001

A. Carter, D. Roques, C. Bristow, P. Kinny 
Understanding Mesozoic accretion in Southeast Asia: signigicance of Triassic thermotectonism (Indosinian orogeny) in Vietnam

Geology, 29 (2001), pp. 211-214

Charvet et al., 1994

J. Charvet, H. Lapierre, Y. Yu

Geodynamics significance of the Mesozoic volcanism of southeastern China

Journal of Southeast Asian Earth Sciences, 9 (1994), pp. 387-396

Charvet et al., 2010

J. Charvet, L. Shu, M. Faure, F. Choulet, B. Wang, H. Lu, N.L. Breton

Structural development of the Lower Paleozoic belt of South China: genesis of an intracontinental orogen

Journal of Asian Earth Sciences, 39 (2010), pp. 309-330

Chen, 1999

A. Chen

Mirror-image thrusting in the South China Orogentic Belt: tectonic evidence from western Fujian, southeastern China

Tectonophysics, 305 (1999), pp. 497-519

Chen and Jahn, 1998

J.F. Chen, B.M. Jahn

Crustal evolution of southeastern China: $\mathrm{Nd}$ and $\mathrm{Sr}$ isotopic evidence

Tectonophysics, 284 (1998), pp. 101-133

Chen and Zhu, 1993

Y.C. Chen, Y.S. Zhu

Mineral Deposits of China

Geological Publishing House, Beijing (1993) pp. 209-211 (in Chinese)

Chen et al., 2000

C.H. Chen, W. Lin, C.Y. Lee, J.L. Tien, H.Y. Lu, Y.H. Lai

Cretaceous fractionated $I$-type granitoids and metaluminous $A$-type granites in SE

China: the Late Yanshanian post-orogenic magmatism

Transaction Royal Society of Edinburgh, Earth Science, 91 (2000), pp. 195-205

Chen et al., 2008

Chen, M.H., Cheng, Y.B., Zhang, W., Yang, Z.X., Lu, G., 2008. Primary Study on the Relationship between Yanshanian Magma Activity and Mineralization in Youjiang folded belt. In: Chen, Y.C., Xue, C.J., Zhang, C.Q. (Eds.), Main Attacking Deep, Pushing Forward Western and Scanning the World. 9th National Deposit Meeting. Geological Publishing House, Beijing, pp. 241-242 (in Chinese).

Chen et al., 2009a

D.Z. Chen, J.G. Wang, H.R. Qing, D.T. Yan, R.W. Li

Hydrothermal venting activities in the Early Cambrian, South China: petrological, geochronological and stable isotopic constraints

Chemical Geology, 258 (2009), pp. 168-181

Chen et al., 2009b 
M.H. Chen, J.W. Mao, W. Zhang, Z.X. Yang, G. Lu, K.J. Hou, J.H. Liu

Zircon $\mathrm{U}-\mathrm{Pb}$ age and $\mathrm{Hf}$ isotopic composition of the Baiceng ultrabasic rock veins in Zhenfeng county, southwestern Guizhou Province, China

Mineral Deposits, 28 (2009), pp. 240-250 (in Chinese with English abstract)

Chen et al., 2011

C.H. Chen, P.S. Hsieh, C.Y. Lee, H.W. Zhou

Two episodes of the Indosinian thermal event on the South China Block:

constraints from LA-ICPMS U-Pb zircon and electron microprobe monazite ages of the Darongshan S-type granitic suite

Gondwana Research, 19 (2011), pp. 1008-1023

Cheng and Mao, 2010

Y.B. Cheng, J.W. Mao

Age and geochemistry of granites in Gejiu area, Yunnan province, SW China: constraints on their petrogenesis and tectonic setting

Lithos, 120 (2010), pp. 258-276

Chu and Lin, this volume

Chu, Y., Lin, W., this volume. Phanerozoic polyorogenic deformation in southern Jiuling massif, northern South China block: constraints from structural analysis and geochronology. Journal of Asian Earth Sciences, in press.

Chu et al., 2002

N.C. Chu, R.N. Taylor, V. Chavagnac, R.W. Nesbitt, R.M. Boella, J.A. Milton, C.R. German, G. Bayon, K. Burton

Hf isotope ratio analysis using multi-collector inductively coupled plasma mass spectrometry: an evaluation of isobaric interference corrections

Journal of Analytical Atomic Spectrometry, 17 (2002), pp. 1567-1574

Chu et al., 2012

Y. Chu, W. Lin, M. Faure, Q.C. Wang, W.B. Ji

Phanerozoic tectonothermal events of the Xuefengshan Belt, central South China: Implications from $\mathrm{U}-\mathrm{Pb}$ age and $\mathrm{Lu}-\mathrm{Hf}$ determinations of granites

Lithos, 150 (2012), pp. 243-255

Chung and Jahn, 1995

S.L. Chung, B.M. Jahn

Plume-lithosphere interaction in generation of the Emeishanflood basalts at the Permian-Triassic boundary

Geology, 10 (1995), pp. 889-892

De Paolo, 1981

D.J. De Paolo

A neodymium and strontium isotopic study of the Mesozoic calcalkaline granitic batholiths of the Sierra Nevada and Peninsular Ranges, California

Journal of Geophysical Research, 86 (1981), pp. 10470-10488

Deng et al., 2004

X.G. Deng, Z.G. Chen, X.H. Li, D.Y. Liu 
SHRIMP U-Pb zircon dating of the Darongshan-Shiwandashan granitoid belt in southeastern Guangxi, China

Geological Review, 50 (2004), pp. 427-432 (in Chinese with English abstract)

Deprat, 1915

Deprat, J., 1915. Etude géologique de la région septentrionale du haut Tonkin (feuille de Pa Kha, Ha Giang, Ma Li Po, Yen Minh). Mémoire du Service Géologique de I'Indochine, Hanoi, vol. IV, 174pp.

Ding et al., 2005

X. Ding, X.M. Zhou, T. Sun

The episodic growth of the continental crustal basement in South Chian: single zircon LA-ICPMS U-Pb dating of Guzhai granodiorite in Guangdong

Geological Review, 51 (2005), pp. 382-392

Dong and Peng, 1994

C.W. Dong, Y.M. Peng

Qingtian composite body-the coexisting of two different types of granties

Journal Zhejiang University (Natural Science), 28 (1994), pp. 440-448 (in Chinese with English abstract)

Eiler, 2001

J.M. Eiler

Oxygen isotope variations of basaltic lavas and upper mantle rocks

Reviews in Mineralogy and Geochemistry, 43 (2001), pp. 319-364

Faure et al., 1996

M. Faure, Y. Sun, L. Shu, P. Monié, J. Charvet

Extensional tectonics within a subduction type orogen: the case study of the

Wugongshan dome (Jiangxi Province, southeastern China)

Tectonophysics, 263 (1996), pp. 77-106

Faure et al., 1999

M. Faure, W. Lin, L.S. Shu, Y. Sun, U. Schärer

Tectonics of the Dabieshan (eastern China) and possible exhumation mechanism of ultra high-pressure rocks

Terra Nova, 11 (1999), pp. 251-258

Faure et al., 2009

M. Faure, L. Shu, B. Wang, J. Charvet, F. Choulet, P. Monié

Intracontinental subduction: a possible mechanism for the Early Palaeozoic

Orogen of SE China

Terra Nova, 21 (2009), pp. 360-368

Faure et al., submitted for publication

Faure, M., Lepvrier, C., Nguyen, V.V., Vu, V.T., Lin, W., Chen, Z.C., submitted for publication. The South China Block-Indochina collision: where, when, and how? Journal of Asian Earth Sciences. 
FBGMR (Fujian Bureau of Geology and Mineral Resources), 1985. Regional Geology of Fujian Province. Geological Publishing House, Beijing, pp. 671.

Fromaget, 1941

J. Fromaget

L'Indochine francaise, sa structure géologique, ses roches, ses mines et leurs relations possibles avec la tectonique

Bulletin Service Géologique de l'Indochine, 26 (1941), pp. 1-140

GDBGMR, 1982

GDBGMR (Guangdong Bureau of Geology and Mineral Resources), 1982. Regional

Geology of Guangdong Province. Geological Publishing House, Beijing, pp. 941.

Gilder et al., 1991

S. Gilder, G.R. Keller, M. Luo

Eastern Asia and the western Pacific timing and spatial distribution of rifting in China

Tectonophysics, 197 (1991), pp. 225-243

Gilley et al., 2003

L.D. Gilley, T.M. Harrison, P.H. Leloup, F.J. Ryerson, O.M. Lovera, J.H. Wang

Direct dating of left-lateral deformation along the Red River shear zone, China and Vietnam

Journal of Geophysical Research, 108 (B2) (2003), p. 2127

doi:10.1029/2001JB001726

GXBGMR, 1982

GXBGMR (Guangxi Bureau of Geology and Mineral Resources), 1982. Regional Geology of Guangdong Province. Geological Publishing House, Beijing, pp. 853.

Hacker et al., 1998

B. Hacker, L. Ratschbacher, L. Webb, T. Ireland, D. Walker, S.W. Dong

$\mathrm{U}-\mathrm{Pb}$ zircon ages constrain the architecture of the ultrahigh-pressure Qinling-

Dabie Orogen, China

Earth and Planetary Science Letters, 161 (1998), pp. 215-230

Hanski et al., 2004

E. Hanski, R.J. Walker, H. Huhma, G.V. Polyakov, P.A. Balykin, T.H. Tran, T.P. Ngo

Origin of the Permian-Triassic komatiites, northwestern Vietnam

Contributions to Mineralogy and Petrology, 147 (2004), pp. 453-469

HBGMR, 1988

HBGMR (Hunan Bureau of Geology and Mineral Resources), 1988. Regional Geology of Hunan Province. Geological Publishing House, Beijing, pp. 726.

He et al., 2011

Z.Y. He, X.S. Xu, Y.L. Niu

Petrogenesis and tectonic significance of a Mesozoic granite-syenite-gabbro association from inland South China 
Lithos, 119 (2011), pp. 621-641

Hou et al., 2007

Z.Q. Hou, K. Zaw, G.T. Pan, X.X. Mo, Q. Xu, Y.Z. Hu, X.Z. Li

Sanjiang Tethyan metallogenesis in S.W. China: tectonic setting, metallogenic epochs and deposit types

Ore Geology Reviews, 31 (2007), pp. 48-87

Huang, 1978

T.K. Huang

An outline of the tectonic characteristics of China

Ecologae Geologicae Helvetiae, 71 (1978), pp. 611-635

Huang et al., 1980

J.Q. Huang, J.S. Ren, C.F. Jiang, Z.K. Zhang, D.Y. Qin

Tectonics and Evolution of China

Science Press House, Beijing (1980) pp. 124 (in Chinese)

JBGMR, 1984

JBGMR (Jiangxi Bureau of Geology and Mineral Resources), 1984, Regional geology of Jiangxi Province. Geological Publishing House, Beijing, pp. 921.

Jian et al., 1999

P. Jian, X.F. Wang, L.Q. He, C.S. Wang

$\mathrm{U}-\mathrm{Pb}$ zircon dating of anothosite and plagiogranite from the Jinshaj iang ophiolite belt

Acta Petrologica Sinica, 15 (1999), pp. 590-593

Jian et al., 2003

P. Jian, D.Y. Liu, X.M. Sun

SHRIMP Dating of Baimaxueshan and Ludian Granitoid Batholiths, Northwestern Yunnan Province, and Its Geological Implications

Acta Geoscientia Sinica, 24 (2003), pp. 337-342

Jiang et al., 2011

Y.H. Jiang, P. Zhao, Q. Zhou, S.Y. Liao, G.D. Jin

Petrogenesis and tectonic implications of Early Cretaceous $S$ - and $A$-type granites in the northwest of the Gan-Hang rift, SE China

Lithos, 121 (2011), pp. 55-73

John et al., 1990

B.M. Jahn, X.H. Zhou, J.L. Li

Formation and tectonic evolution of southeastern China and Taiwan: isotopic and geochemical constraints

Tectonophysics, 183 (1990), pp. 145-160 
Lan et al., 2003

C.Y. Lan, S.L. Chung, V.L. Trinh, C.H. Lo, T.Y. Lee, S.A. Mertzman, J. Shen

Geochemical and $\mathrm{Sr}-\mathrm{Nd}$ isotopic constraints from the Kontum massif, central

Vietnam on the crustal evolution of the Indochina block

Precambrian Research, 122 (2003), pp. 7-27

Leech and Webb, 2012

M.L. Leech, L.E. Webb

Is the HP-UHP Hong'an-Dabie-Sulu orogen a piercing point for offset on the TanLu fault?

Journal of Asian Earth Sciences, 63 (2012), pp. 112-129

Leloup et al., 1995

P.H. Leloup, R. Lacassin, P. Tapponnier, U. Schärer, D.L. Zhong, X.H. Liu, L.S. Zhang, S.C. Ji, T.T. Phan

The Ailo Shan-Red River shear zone (Yunnan, China), Tertiary transform

boundary of Indochina

Tectonophysics, 252 (1995), pp. 3-84

Lepvrier et al., 1997

C. Lepvrier, H. Maluski, V.V. Nguyen, D. Roques, V. Axente, G. Rangin

Indosinian NW-trending shear zones within the Truong Son belt (Vietnam)

Tectonophysics, 283 (1997), pp. 105-127

Lepvrier et al., 2004

C. Lepvrier, H. Maluski, V.T. Vu, A. Leyreloup, T.T. Phan, V.V. Nguyen

The Early Triassic Indosinian orogeny in Vietnam (Truong Son Belt and Kontum Massif): implications for the geodynamic evolution of Indochina

Tectonophysics, 393 (2004), pp. 87-118

Lepvrier et al., 2008

C. Lepvrier, V.V. Nguyen, H. Maluski, T.T. Phan, V.T. Vu

Indosinian tectonics in Vietnam

Comptes Rendus Geosciences, 340 (2008), pp. 94-111

Lepvrier et al., 2011

C. Lepvrier, M. Faure, V.V. Nguyen, V.T. Vu, W. Lin, T.T. Ta, H.P. Ta

North-directed Triassic nappes in Northeastern Vietnam (East Bac Bo)

Journal of Asian Earth Sciences, 41 (2011), pp. 56-68

Li, 1994

X. Li

A comprehensive $\mathrm{U} / \mathrm{Pb}, \mathrm{Sm} / \mathrm{Nd}, \mathrm{Rb} / \mathrm{Sr}$ and ${ }^{40} \mathrm{Ar} /{ }^{39} \mathrm{Ar}$ geochronological study on Guidong granodiorite, southeast China: records of multiple tectonothermal events in a single pluton

Chemical Geology, 115 (1994), pp. 283-295

$\mathrm{Li}, 1996$

X.H. Li

A discussion on the model and isochron ages of $\mathrm{Sm}-\mathrm{Nd}$ isotopic systematics:

suitability and limitation 
Scientia Geologica Sinica, 31 (1996), pp. 97-104

Li, 1999

X.H. Li

$\mathrm{U}-\mathrm{Pb}$ zircon ages of granites from the southern margin of the Yangtze margin:

Timing of Neoproterozoic Jinning Orogen in SE China and implication for

Rodinia assembly

Precambrian Research, 97 (1999), pp. 43-57

Li, 2000

X.H. Li

Cretaceous magmatism and lithosphere extension in Southeast China

Jounranl of Asian Earth Sciences, 18 (2000), pp. 293-305

$\mathrm{Li}$ and $\mathrm{Li}, 2007$

Z.X. Li, X.H. Li

Formation of the $1300 \mathrm{~km}$ wide intracontinental orogen and postorogenic magmatic province in Mesozoic south China: a flat-slab subduction model Geology, 35 (2007), pp. 179-182

Li et al., 2001

J.W. Li, M.F. Zhou, X.F. Li, Z.R. Fu, Z.J. Li

The Hunan-Jiangxi strike-slip fault system in southern China: Southern termination of the Tan-Lu fault

Journal of Geodynamics, 32 (2001), pp. 333-354

Li et al., 2004

Li, X.H., Chung, S.L., Zhou, H.W., Lo, C.H., Liu, Y., Chen, C.H., 2004. Jurassic intraplate magmatism in southern Hunan-eastern Guangxi: ${ }^{40} \mathrm{Ar} /{ }^{39} \mathrm{Ar}$ dating, geochemistry, $\mathrm{Sr}-\mathrm{Nd}$ isotopes and implications for tectonic evolution of SE China. In: Malpas, J., Fletcher, C.J., Aitchison, J.C., Ali, J. (Eds.), Aspects of the Tectonic Evolution of China. Geological Society of London. Special Publication, vol. 226, pp. 193-216.

Li et al., 2006

X.H. Li, Z.X. Li, W.X. Li, Y.J. Wang

Initiation of the Indosinian Orogeny in South China: evidence for a Permian magmatic arc on Hainan Island

Journal of Geology, 114 (2006), pp. 341-353

Li et al., 2009a

X.H. Li, W.X. Li, Z.X. Li, C.H. Lo, J. Wang, M.F. Ye, Y.H. Yang

Amalgamation between the Yangtze and Cathaysia Blocks in South China:

constraints from SHRIMP U-Pb zircon ages, geochemistry and $\mathrm{Nd}-\mathrm{Hf}$ isotopes of the Shuangxiwu volcanic rocks

Precambrian Research, 174 (2009), pp. 117-128

Li et al., 2009b

X.H. Li, Y. Liu, Q.L. Li, C.H. Guo, K.R. Chamberlain 
Precise determination of Phanerozoic zircon $\mathrm{Pb} / \mathrm{Pb}$ age by multicollector SIMS without external standardization

Geochemistry, Geophysics, Geosystems, 10 (2009), p. Q04010

http://dx.doi.org/10.1029/2009GC002400

Li et al., 2010a

Q.L. Li, X.H. Li, Y. Liu, G.Q. Tang, J.H. Yang, W.G. Zhu

Precise $\mathrm{U}-\mathrm{Pb}$ and $\mathrm{Pb}-\mathrm{Pb}$ dating of Phanerozoic baddeleyite by SIMS with oxygen flooding technique

Journal of Analytical Atomic Spectrometry, 25 (2010), pp. 1107-1113

Li et al., 2010b

Q.L. Li, X.H. Li, Y. Liu, F.Y. Wu, J.H. Yang, R.H. Mitchell

Precise $\mathrm{U}-\mathrm{Pb}$ and $\mathrm{Th}-\mathrm{Pb}$ age determination of kimberlitic perovskites by

secondary ion mass spectrometry

Chemical Geology, 269 (2010), pp. 396-405

Li et al., 2010c

X.H. Li, W.G. Long, Q.L. Li, Y. Liu, Y.F. Zheng, Y.H. Yang, K.R. Chamberlain, D.F. Wan, C.H. Guo, X.C. Wang, H. Tao

Penglai zircon megacrysts: a potential new working reference material for microbeam determination of $\mathrm{Hf}-\mathrm{O}$ isotopes and $\mathrm{U}-\mathrm{Pb}$ age

Geostandards and Geoanalytical Research, 34 (2010), pp. 117-134

Li et al., 2010d

Z.X. Li, X.H. Li, J.A. Wartho, C. Clark, W.X. Li, C.L. Zhang, C. Bao

Magmatic and metamorphic events during the early Paleozoic Wuyi-Yunkai orogeny, southeastern South China: new age constraints and pressure-temperature conditions

Geologyical Society of America Bulletin, 122 (2010), pp. 772-793

Li et al., 2012

W. Li, C. Ma, Y. Liu, P. Robinson

Discovery of the Indosinian aluminum A-type granite in Zhejiang Province and its geological significance

Science China Earth Sciences, 55 (2012), pp. 13-25

Liang et al., 2008

T. Liang, Y.C. Chen, D.H. Wang, M.H. Cai

Geology and Geochemistry of the Dachang Tin Polymetallic Deposit, Guangxi

ProvinceGeological Publishing House, Beijing (2008) pp. 1-229 (in Chinese)

Liew and Hofmann, 1988

T.C. Liew, A.W. Hofmann

Precambrian crustal components, plutonic assimilations, plate environment of the Hercynian fold belt of central Europe: indications from a $\mathrm{Nd}$ and $\mathrm{Sr}$ isotopic study Contributions to Mineralogy and Petrology, 98 (1988), pp. 129-138

Lin et al., 2000

W. Lin, M. Faure, P. Monié, U. Schäerer, L. Zhang, Y. Sun 
Tectonics of SE China: new insights from the Lushan massif (Jiangxi Province) Tectonics, 19 (2000), pp. 852-871

Lin et al., 2008

W. Lin, Q.C. Wang, K. Chen

Phanerozoic tectonics of south China block: new insights from the polyphase deformation in the Yunkai massif

Tectonics, 27 (2008), p. TC6004 doi:10.1029/2007TC002207

Lin et al., 2011

W. Lin, M. Faure, C. Lepvrier, Z.C. Chen, Y. Chu, Q.C. Wang, V.V. Nguyen, V.T. Vu

The Early Mesozoic thrust and folds sheet structure along the southern margin of South China Block and its geodynamic

Scientia Geologica Sinica, 46 (2011), pp. 134-145 (in Chinese with English abstract)

Liu et al., 2007

Y.P. Liu, Z.X. Li, H.M. Li

$\mathrm{U}-\mathrm{Pb}$ geochronology of cassiterite and zircon from the Dulong $\mathrm{Sn}-\mathrm{Zn}$ deposit: evidence for Cretaceous large — scale granitic magmatism and mineralization events in southeastern Yunnan province, China

Acta Petrologica Sinica, 23 (2007), pp. 967-976 (in Chinese with English abstract)

Liu et al., 2010

S. Liu, W.C. Su, R.Z. Hu, C.X. Feng, S. Gao, I.M. Coulson, T. Wang, G.Y. Feng, Y. Tao, Y. Xia Geochronological and geochemical constraints on the petrogenesis of alkaline ultramafic dykes from southwest Guizhou Province, SW China

Lithos, 114 (2010), pp. 253-264

Liu et al., 2012

J.L. Liu, M.D. Tran, Y. Tang, Q.L. Nguyen, T.H. Tran, W.B. Wu, J.F. Chen, Z.C. Zhang, Z.D. Zhao

Permo-Triassic granitoids in the northern part of the Truong Son belt, NW

Vietnam: Geochronology, geochemistry and tectonic implications

Gondwana Research, 22 (2012), pp. 628-644

Ludwig, 2001

Ludwig, K.R., 2001. Users manual for Isoplot/Ex rev. 2.49. Berkeley Geochronology

Centre Special, Publication. No. 1a, pp. 56.

Lugmair and Marti, 1978

G.W. Lugmair, K. Marti

Lunar initial ${ }^{143} \mathrm{Nd} /{ }^{144} \mathrm{Nd}$ : differential evolution of the lunar crust and mantle

Earth and Planetary Science Letters, 39 (1978), pp. 349-357

Maluski et al., 2001

H. Maluski, C. Lepvrierb, L. Jolivetb, A. Carterc, D. Roquesc, O. Beyssacd

$\mathrm{Ar}-\mathrm{Ar}$ and fission-track ages in the Song Chay Massif: early Triassic and

Cenozoic tectonics in northern Vietnam

Journal of Asian Earth Sciences, 19 (2001), pp. 233-248 
Martin et al., 1994

H. Martin, B. Bonin, R. Capdevila, B.M. Jahn, J. Lameyre, Y. Wang

The Kuiqi peralkaline granitic complex (SE China): petrology and geochemistry Journal of Petrology, 35 (1994), pp. 983-1015

Metcalfe, 2002

I. Metcalfe

Permian tectonic framework and palaeogeography of SE Asia

Journal of Asian Earth Science, 20 (2002), pp. 551-566

Nagy et al., 2001

E.A. Nagy, H. Maluski, C. Lepvrier, U. Schärer, T.T. Phan, A. Leyreloup, V.T. Vu

Geodynamic significance of the Kontum massif in central Vietnam: composite

${ }^{40} \mathrm{Ar} /{ }^{39} \mathrm{Ar}$ and $\mathrm{U}-\mathrm{Pb}$ ages from Paleozoic to Triassic

Journal of Geology, 109 (2001), pp. 755-770

Nakano et al., 2008

N. Nakano, Y. Osanai, T.M. Nguyen, T. Miyamoto, Y. Hayasaka, M. Owada

Discovery of high-pressure granulite-facies metamorphism in northern Vietnam: constraints on the Permo-Triassic Indochinese continental collision tectonics Comptes Rendus Geosciences, 340 (2008), pp. 127-139

Osanai et al., 2001

Y. Osanai, M. Owada, T. Tsunogae, T. Toyoshima, T. Hokada, V.L. Trinh, K. Sajeev, N. Nakano

Ultra high-temperature pelitic granulites from Kontum massif, central Vietnam: evidence for East Asian juxtaposition at ca. $250 \mathrm{Ma}$

Gondwana Research, 4 (2001), pp. 720-723

Osanai et al., 2006

Y. Osanai, M. Owada, A. Kamei, T. Hamamto, H. Kagami, T. Toyoshima, H. Nakano, N.N. Tran

The Higo metamorphic complex in Kyushu, Japan as the fragment of Permo-

Triassic metamorphic complexes in East Asia

Gondwana Research, 9 (2006), pp. 152-166

Phan et al., 2012

T.T. Phan, V.L. Ngo, V.H. Nguyen, Q.V. Hoang, V.T. Bui, T.T. Bui, T.T. Mai, H. Nguyen Late Quaternary tectonics and seismotectonics along the Red River fault zone, North Vietnam

Earth-Science Reviews, 114 (2012), pp. 224-235

Qi et al., 2007

C.S. Qi, X.G. Deng, W.X. Li, X.H. Li, Y.H. Yang, L.W. Xie

Origin of the Darongshan-Shiwandashan $S$-type granitoid belt from southeastern Guangxi: geochemicaland $\mathrm{Sr}-\mathrm{Nd}-\mathrm{Hf}$ isotopic constraints

Acta Petrologica Sinica, 2 (2007), pp. 403-412 
Qiu et al., 2008

J.S. Qiu, E. Xiao, J. Hu, X.S. Xu, S.Y. Jiang, Z. Li

Petrogenesis of highly fractionated I-type granites in the coastal area of northeastern Fujian Province: constraints from zircon $\mathrm{U}-\mathrm{Pb}$ geochronology, geochemistry and $\mathrm{Nd}-\mathrm{Hf}$ isotopes

Acta Petrologica Sinica, 24 (2008), pp. 2468-2484

Roger et al., 2000

F. Roger, P.H. Leloup, M. Jolivet, R. Lacassin, T.T. Phan, M. Brunel, D. Seward

Long and complex thermal history of the Song Chay metamorphic dome (Northern Vietnam) by multi-system geochronology

Tectonophysics, 321 (2000), pp. 449-466

Roger et al., 2010

F. Roger, M. Jolivet, J. Malavieille

The tectonic evolution of the Songpan-Garze (North Tibet) and adjacent areas from Proterozoic to Present: a synthesis

Journal of Asian Earth Sciences, 39 (2010), pp. 254-269

Roger et al., 2012

F. Roger, H. Maluski, C. Lepvrier, V.T. Vu, J.L. Paquette

LA-ICPMS zircons U/Pb dating of Permo-Triassic and Cretaceous magmatisms in Northern Vietnam-Geodynamical implication

Journal of Asian Earth Sciences, 48 (2012), pp. 72-82

Sengör et al., 1988

A.M.C. Sengör, D. Altiner, A. Cin, T. Ustaomer, K.J. Hsu

Origin and assembly of the Tethyside orogenic collage at the expense of

Gondwana Land

Geological Society Special Publication, 37 (1988), pp. 119-181

Shen et al., 1998

W.Z. Shen, H.F. Ling, W.X. Li, D.Z. Wang

$\mathrm{Sr}$ and $\mathrm{Nd}$ isotope of Mesozoic granitoids in Jiangxi Province

Chinese Science Bulletin, 43 (1998), pp. 2653-2657

Shu et al., 1994

L. Shu, G. Zhou, Y. Shi, J. Yin

A study on the high pressure metamorphic blueschist and its late Proterozoic age in the Eastern Jiangnan belt

Chinese Science Bulletin, 39 (1994), pp. 1200-1204

Shu et al., 2008a

L.S. Shu, M. Faure, B. Wang, X.M. Zhou, B. Song

Late Palaeozoic-Early Mesozoic geological features of South China: response to the Indosinian collision events in Southeast Asia

Comptes Rendus Geosciences, 340 (2008), pp. 151-165

Shu et al., $2008 \mathrm{~b}$

L.S. Shu, J.H. Yu, D. Jia, B. Wang, W. Shen, Y.Q. Zhang

Early Palaeozoic orogenic belt in the eastern segment of South China 
Geological Bulletin of China, 27 (2008), pp. 1581-1593

Shui, 1987

T. Shui

Tectonic framework of the continental basement of southeast China

Scientia Sinica (B), 31 (1987), pp. 24-34 (in Chinese)

Sláma et al., 2008

J. Sláma, J. Košler, D.J. Condon, J.L. Crowley, A. Gerdes, J.M. Hanchar, M.S.A. Horstwood, G.A. Morris, L. Nasdala, N. Norberg, U. Schaltegger, B. Schoene, M.N. Tubrett, M.J. Whitehouse

Plešovice zircon - a new natural reference material for $\mathrm{U}-\mathrm{Pb}$ and $\mathrm{Hf}$ isotopic microanalysis

Chemical Geology, 249 (2008), pp. 1-35

Söderlund et al., 2004

U. Söderlund, P.J. Patchett, J.D. Vervoort, C.E. Isachsen

The ${ }^{176} \mathrm{Lu}$ decay constant determined by $\mathrm{Lu}-\mathrm{Hf}$ and $\mathrm{U}-\mathrm{Pb}$ isotope systematics of Precambrian mafic intrusions

Earth and Planetary Science Letters, 219 (2004), pp. 311-324

Sone and Metcalfe, 2008

M. Sone, I. Metcalfe

Parallel Tethyan sutures in mainland Southeast Asia: new insights for Palaeo-

Tethys closure and implications for the Indosinian orogeny

Comptes Rendus Geoscience, 340 (2008), pp. 166-179

Song et al., 2007

S. Song, J. Ji, C. Wei, L. Su, Y. Zheng, B. Song, L. Zhang

Early Paleozoic granite in Nujiang River of northwest Yunnan in southwestern

China and its tectonic implications

Chinese Science Bulletin, 52 (2007), pp. 2402-2406

Stacey and Kramers, 1975

J.S. Stacey, J.D. Kramers

Approximation of terrestrial lead isotope evolution by a two-stage model

Earth and Planetary Science Letters, 26 (1975), pp. 207-221

Steiger and Jäger, 1977

R.H. Steiger, E. Jäger

Subcommission on geochronology; convention on the use of decay constants in geochronology and cosmochronology

Earth and Planetary Science Letters, 36 (1977), pp. 359-362

Sun et al., 2004

T. Sun, X.M. Zhou, P.R. Chen, H.M. Li, H.Y. Zhou, Z.C. Wang, W.Z. Shen

Strongly peraluminous granites of Mesozoic in eastern Nanling Range, Southern

China: petrogenesis and implications for tectonics

Science in China (Series D), 48 (2004), pp. 165-174 
Sun et al., 2011

Y. Sun, C.Q. Ma, Y.Y. Liu, Z.B. She

Geochronological and geochemical constraints on the petrogenesis of late Triassic aluminous $A$-type granites in southeast China

Journal of Asian Earth Sciences, 42 (2011), pp. 1117-1131

Suo et al., 1999

S.T. Suo, X.H. Bi, H.W. Zhou

Very Low Grade Metamorphism

Geological Publishing House, Beijing (1999) pp. 1-68

Tan et al., 2008

J. Tan, J.H. Wei, S.R. Li

Geochemical characteristics and tectonic significance of Kunlunguan A-type

granite, Guangxi

Earth Science-Journal of China University of Geosciences, 33 (2008), pp. 743-754

Tapponnier et al., 1990

P. Tapponnier, R. Lacassin, H. Lelaup, U. Scharer, D.L. Zhong, H.W. Wu, X.H. Liu, S.C. Ji, L.S. Zhang, J.Y. Zong

The Ailao Shan/Red River metamorphic belt: tertiary left-lateral shear between Indochina and South China

Nature, 342 (1990), pp. 431-437

Tran and Halpin, 2011

Tran, Hai Thanh, Halpin, J., 2011. Structural features of magamatic complexes in Ngan Son area, Bac Kan province and their significance in regional tectonic framework. International Workshop on Advanced Research in Geosciences in Asia, Hanoi.

Tran et al., 2008

T.H. Tran, A.E. Izokh, G.V. Polyakov, A.S. Borisenko, T.A. Tran, P.A. Balykin, T.P. Ngo, S.N. Rudnev, V.V. Vu, A.N. Bui

Permo-Triassic magmatism and metallogeny of Northern Vietnam in relation to the Emeishan plume

Russian Geology and Geophysics, 49 (2008), pp. 480-491

Valley et al., 1998

J.W. Valley, P.D. Kinny, D.J. Schulze, M.J. Spicuzza

Zircon megacrysts from kimberlite: oxygen isotope variability among mantle melts Contributions to Mineralogy and Petrology, 133 (1998), pp. 1-11

Valley et al., 2005

J.W. Valley, J.S. Lackey, A.J. Cavosie, C.C. Clechenko, M.J. Spicuzza, M.A.S. Basei, I.N.

Bindeman, V.P. Ferreira, A.N. Sial, E.M. King, W.H. Peck, A.K. Sinha, C.S. Wei

4.4 billion Years of crustal maturation: oxygen isotope ratios of magmatic zircon

Contributions to Mineralogy and Petrology, 150 (2005), pp. 561-580

Van Tri, 1979 
Van Tri

Geology of Vietnam: The Northern Part

Science and Technique Publishing House, Hanoi (1979) pp. 80

Wan et al., 2010

Y.S. Wan, D.Y. Liu, S.M. Wilde, J.J. Cao, B. Chen, C.Y. Dong, B. Song, L.L. Du

Evolution of the Yunkai Terrane, South China: evidence from SHRIMP zircon U$\mathrm{Pb}$ dating, geochemistry and $\mathrm{Nd}$ isotope

Journal of Asian Earth Sciences, 37 (2010), pp. 140-153

Wang and Li, 2003

J. Wang, Z.X. Li

History of Neoproterozoic rift basins in South China: implications for Rodinia break-up

Precambrian Research, 122 (2003), pp. 141-158

Wang et al., 2000

X.F. Wang, I. Metcalfe, P. Jian, L.Q. He, C.S. Wang

The Jinshajiang-Ailaoshan Suture Zone, China: tectonostratigraphy, age and evolution

Journal of Asian Earth Sciences, 18 (2000), pp. 675-690

Wang et al., 2001

D.Z. Wang, L.S. Shu, M. Faure, W.Z. Shen

Mesozoic magmatism and granitic dome in the Wugongshan massif, Jiangxi

Province and their genetical relationship to the tectonic events in southeast China

Tectonophysics, 339 (2001), pp. 259-277

Wang et al., 2002

Y.J. Wang, Y. Zhang, W.M. Fan, X.W. Xi, F. Guo, G. Lin

Numerical modeling of the formation of Indosinian peraluminous granitoids in

Hunan Province: basaltic underplating versus tectonic thickening

Science in China (Series D), 11 (2002), pp. 491-499

Wang et al., 2005

Q. Wang, J.W. Li, P. Jian, Z.H. Zhao, X.L. Xiong, Z.W. Bao, J.F. Xu, C.F. Li, J.L. Ma

Alkaline syenites in eastern Cathaysia (South China): link to Permian-Triassic transtension

Earth and Planetary Science Letters, 230 (2005), pp. 339-354

Wang et al., 2007a

Wang, Y.J., Fan, W.M., Cawood, P.A., Ji, S.C., Peng, T.P., Chen, X.Y., 2007a. Indosinian high-strain deformation for the Yunkaidashan tectonic belt, South China: Kinematics and ${ }^{40} \mathrm{Ar} /{ }^{39} \mathrm{Ar}$ geochronological constraints. Tectonics 26, TC6008, doi: 10.1029/2007TC002099.

Wang et al., 2007b

Y.J. Wang, W.M. Fan, M. Sun, X.Q. Liang, Y.H. Zhang, T.P. Peng 
Geochronological, geochemical and geothermal constraints on petrogenesis of the Indosinian peraluminous granites in the South China Block: a case study in the Hunan Province

Lithos, 96 (2007), pp. 475-502

Wang et al., 2007c

Y.J. Wang, W.M. Fan, G.C. Zhao, S.C. Ji, T.P. Peng

Zircon $\mathrm{U}-\mathrm{Pb}$ geochronology of gneissic rocks in the Yunkai massif and its implications on the Caledonian event in the South China Block

Gondwana Research, 12 (2007), pp. 404-416

Wang et al., 2010

Wang, Y.J., Zhang, F.F., Fan, W.M., Zhang, G.W., Chen, S.Y., 2010. Tectonic setting of the South China Block in the early Paleozoic: Resolving intracontinental and ocean closure models from detrital zircon $\mathrm{U}-\mathrm{Pb}$ geochronology. Tectonics 29, TC6020, doi: 10.1029/2010TC002750, 2010.

Wang et al., 2011

Y.J. Wang, A.M. Zhang, W.M. Fan, G.W. Zhang, F.F. Zhang, Y.Z. Zhang, S.Z. Li

Kwangsian crustal anatexis within the eastern South China Block: geochemical, zircon $\mathrm{U}-\mathrm{Pb}$ geochronological and $\mathrm{Hf}$ isotopic fingerprints from the gneissoid granites of Wugong and Wuyi-Yunkai

Lithos, 127 (2011), pp. 239-260

Wang et al., 2012a

Y. Wang, W. Fan, G. Zhang, Y. Zhang

Phanerozoic tectonics of the South China Block: key observations and controversies

Gondwana Research (2012) http://dx.doi.org/10.1016/j.gr.2012.02.019

Wang et al., 2012b

Y.J. Wang, C.M. Wu, A.M. Zhang, W.M. Fan, Y.H. Zhang, Y.Z. Zhang, T.P. Peng, C.Q. Yin Kwangsian and Indosinian reworking of the eastern South China Block:

constraints on zircon $\mathrm{U}-\mathrm{Pb}$ geochronology and metamorphism of amphibolites and granulites

Lithos, 150 (2012), pp. 227-242

Wei et al., this volume

Wei, W., Chen, Y., Faure, M., Shi, Y.H., Martelet, G., Huo, Q.L., Lin, W., Le Breton, N., Wang, Q.C., this volume. A multidisciplinary study on the emplacement mechanism of the Qingyang-Jiuhua Massif in Southeast China and its tectonic bearings. Part I: structural geology, AMS and paleomagnetism. Journal of Asian Earth Sciences, in press.

Wiedenbeck et al., 1995

M. Wiedenbeck, P. Alle, F. Corfu, W.L. Griffin, M. Meier, F. Oberli, A. Vonquadt, J.C. Roddick, W. Speigel

Three natural zircon standards for $\mathrm{U}-\mathrm{Th}-\mathrm{Pb}, \mathrm{Lu}-\mathrm{Hf}$, trace-element and $\mathrm{REE}$ analyses 
Geostandards Newsletter, 19 (1995), pp. 1-23

Wiedenbeck et al., 2004

M. Wiedenbeck, J.M. Hanchar, W.H. Peck, P. Sylvester, J. Valley, M. Whitehouse, A.

Kronz, Y. Morishita, L. Nasdala, J. Fiebig, I. Franchi, J.P. Girard, R.C. Greenwood, R.

Hinton, N. Kita, P.R.D. Mason, M. Norman, M. Ogasawara, P.M. Piccoli, D. Rhede, H.

Satoh, D. Schulz-Dobrik, O. Skar, M.J. Spicuzza, K. Terada, A. Tindle, S. Togashi, T.

Vennemann, Q. Xie, Y.F. Zheng

Further characterization of the 91,500 zircon crystal

Geostand Geoanalytical Research, 28 (2004), pp. 9-39

Wu et al., 2006a

F.Y. Wu, Y.H. Yang, L.W. Xie, J.H. Yang, P. Xu

$\mathrm{Hf}$ isotopic compositions of the standard zircons and baddeleyites used in $\mathrm{U}-\mathrm{Pb}$ geochronology

Chemical Geology, 234 (2006), pp. 105-126

Wu et al., 2006b

R. Wu, Y. Zheng, Y. Wu, Z. Zhao, S. Zhang, X. Liu, F. Wu

Reworking of juvenile crust: element and isotope evidence from Neoproterozoic granodiorite in south China

Precambrian Research, 146 (2006), pp. 179-212

Xie et al., 2006

C.F. Xie, J.C. Zhu, S.J. Ding, Y.M. Zhang, M.L. Chen, Y.R. Fu, T.A. Fu, Z.H. Li

Age and petrogenesis of the Jianfengling granite and its relationship to metallogenesis of the Baolun gold deposit, Hainan Island

Acta Petrologica Sinica, 22 (2006), pp. 2493-2508

Xu et al., 1987

J. Xu, G. Zhu, W. Tong, K. Cui, Q. Liu

Formation and evolution of the Tancheng-Lujiang wrench fault system: a major shear system to the northwest Pacific Ocean

Tectonophysics, 134 (1987), pp. 273-310

Xu et al., 2001

Y.G. Xu, S.L. Chung, B.M. Jahn, G.Y. Wu

Petrologic and geochemical constraints on the petrogenesis of Permian-Triassic Emeishan flood basalts in southwestern China

Lithos, 58 (2001), pp. 145-168

Xu et al., 2004

Y.G. Xu, B. He, S.L. Chung, M.A. Menzies, F.A. Frey

Geologic, geochemical, and geophysical consequences of plume involvement in the Emeishan flood-basalt province

Geology, 10 (2004), pp. 917-920

Yan et al., 2003

D.P. Yan, M.F. Zhou, H.L. Song, X.W. Wang, J. Malpas

Origin and tectonic significance of a Mesozoic multi-layer over-thrust system within the Yangtze block (south China) 
Tectonophysics, 361 (2003), pp. 239-254

Yan et al., 2005

D.P. Yan, M.F. Zhou, Y. Wang, C.L. Wang, T.P. Zhao

Structural styles and chronological evidences from Dulong-Song Chay tectonic dome: earlier spreading of south China sea basin due to late mesozoic to early cenozoic extension of south China block

Earth Science-Journal of China University of Geosciences, 30 (2005), pp. 402-412

Yan et al., 2006

D.P. Yan, M.F. Zhou, C.Y. Wang, B. Xia

Structural and geochronological constraints on the tectonic evolution of the

Dulong-Song Chay tectonic dome in Yunnan province, SW China

Journal of Asian Earth Sciences, 208 (2006), pp. 332-353

Yan et al., 2011

D.P. Yan, M.F. Zhou, S.B. Li, G.Q. Wei

Structural and geochronological constraints on the Mesozoic-Cenozoic tectonic evolution of the Longmen Shan thrust belt, eastern Tibetan Plateau

Tectonics, 30 (2011), p. TC6005 doi: 10.1029/2011TC002867.

Yang and Besse, 1993

Z. Yang, J. Besse

Paleomagnetic study on Permian and Mesozoic sedimentary rocks from North Thailand supports the extrusion model for Indochina

Earth and Planetary Science Letters, 117 (1993), pp. 525-552

Yu et al., 2006

J. Yu, W. Shen, L. Wang, L. Shu, T. Sun

Cathaysia block: a young continent composed of ancient materials

Geological Journal of China Universities, 12 (2006), pp. 440-447

Yu et al., 2007

J. Yu, Y.S. O Reilly, L. Wang, W.L. Griffin, S. Jiang, Y. Wang, X. Xu

Finding of ancient materials in Cathaysia and implication for the formation of

Precambrian crust

Chinese Science Bulletin, 52 (2007), pp. 11-18

ZBGMR, 1989

ZBGMR (Zhejiang Bureau of Geology and Mineral Resources), 1989. Regional Geology of

Zhejiang Zejian Province. Geological Publishing House, Beijing, pp. 688.

Zhang et al., 1984

Z.M. Zhang, J.G. Liou, R.G. Coleman

An outline of the plate tectonics of China

Geologyical Society of America Bulletin, 95 (1984), pp. 295-312

Zhang et al., 2008 
X.H. Zhang, H.F. Zhang, Y.J. Tang, S.A. Wilde, Z.C. Hu

Geochemistry of Permian bimodal volcanic rocks from Central Inner Mongolia, North China: implication for Tectonic setting and Phanerozoic continental growth in Central Asian Orogenic Belt

Chemical Geology, 249 (2008), pp. 262-281

Zhang et al., 2011

F.F. Zhang, Y.J. Wang, X.Y. Chen, W.M. Fan, Y.H. Zhang, A.M. Zhang

Triassic high-strain shear zones in Hainan Island (South China) and their implications on the amalgamation of the Indochina and South China Blocks: Kinematic and ${ }^{40} \mathrm{Ar} /{ }^{39} \mathrm{Ar}$ geochronological constraints

Gondwana Research, 19 (2011), pp. 910-925

Zhao et al., 2010

L. Zhao, F. Guo, W.M. Fan, C.W. Li, X.F. Qin, H.X. Li

Crustal evolution of the Shiwandashan area in South China: Zircon U-Pb-Hf isotopic records from granulite enclaves in Indo-Sinian granites

Chinese Science Bulletin, 55 (2010), pp. 2028-2038

Zhou and Li, 2000

X.M. Zhou, W.X. Li

Origin of Late Mesozoic igneous rocks in Southeastern China: implications for lithosphere subduction and underplating of mafic magmas

Tectonophysics, 326 (2000), pp. 269-287

Zhou and Zhu, 1993

X.M. Zhou, Y.H. Zhu

Petrologic data from the Late Proterozoic collision-orogenic belt and suture zone in the southeast of China

J.L. Li (Ed.), Crustal Structure and Geological Evolution of Southeast Continents, Publishing House of Metallurgy and Industry, Beijing (1993), pp. 87-97

Zhou et al., 2006

X. Zhou, T. Sun, W. Shen, L. Shu, Y. Niu

Petrogenesis of Mezozoic granitoids and volcanic rocks in south China: a response to tectonic evolution

Episodes, 29 (2006), pp. 26-33 